\title{
Continuity equations and ODE flows with non-smooth velocity*
}

\author{
Luigi Ambrosio
}

Scuola Normale Superiore, Piazza Cavalieri 7, 56126 Pisa, Italy

(1.ambrosio@sns.it)

\section{Gianluca Crippa}

Departement Mathematik und Informatik, Universität Basel, Mathematisches Institut, Rheinsprung 21, 4051 Basel, Switzerland (gianluca.crippa@unibas.ch)

(MS received 4 November 2013; accepted 28 April 2014)

\begin{abstract}
In this paper we review many aspects of the well-posedness theory for the Cauchy problem for the continuity and transport equations and for the ordinary differential equation $(\mathrm{ODE})$. In this framework, we deal with velocity fields that are not smooth, but enjoy suitable 'weak differentiability' assumptions. We first explore the connection between the partial differential equation (PDE) and the ODE in a very general non-smooth setting. Then we address the renormalization property for the PDE and prove that such a property holds for Sobolev velocity fields and for bounded variation velocity fields. Finally, we present an approach to the ODE theory based on quantitative estimates.
\end{abstract}

\section{Introduction}

In this paper (based in part on [10,11], with additional updates and recent improvements) we study the well-posedness of the Cauchy problem for the homogeneous conservative continuity equation

$$
\frac{\mathrm{d}}{\mathrm{d} t} \mu_{t}+\mathrm{D}_{x} \cdot\left(\boldsymbol{b} \mu_{t}\right)=0, \quad(t, x) \in I \times \mathbb{R}^{d}
$$

for time-dependent families $\mu_{t}$ of probability measures in $\mathbb{R}^{d}$ (or, more generally, signed measures) and for the transport equation

$$
\frac{\mathrm{d}}{\mathrm{d} t} w_{t}+\boldsymbol{b} \cdot \nabla w_{t}=c_{t}
$$

Here, $\boldsymbol{b}(t, x)=\boldsymbol{b}_{t}(x)$ is a given time-dependent vector field in $\mathbb{R}^{d}$ and $I \subset \mathbb{R}$ is an interval: we are interested in the case in which $\boldsymbol{b}_{t}(\cdot)$ is not necessarily Lipschitz and has, for instance, a Sobolev or bounded variation (BV) regularity. Vector fields with

\footnotetext{
* This paper is a late addition to the papers surveying active areas in partial differential equations, published in issues 141.2 and 142.6, which were based on a series of mini-courses held in Edinburgh from 2010 to 2013.
}

(C) 2014 The Royal Society of Edinburgh 
this 'low' regularity show up, for instance, in several partial differential equations (PDEs) describing the motion of fluids and in the theory of conservation laws.

We are also particularly interested in the well-posedness of the system of ordinary differential equations (ODEs)

$$
\left.\begin{array}{rl}
\dot{\gamma}(t) & =\boldsymbol{b}_{t}(\gamma(t)) \\
\gamma(0) & =x
\end{array}\right\}
$$

In some situations one might hope for a 'generic' uniqueness of the solutions of the system of ODEs, i.e. for 'almost every (a.e.)' initial datum $x$. An even weaker requirement is the research of a 'selection principle', i.e. a strategy to select, for $\mathcal{L}^{d}$-a.e. $x$, a solution $\boldsymbol{X}(\cdot, x)$ in such a way that this selection is stable with respect to (w.r.t.) smooth approximations of $\boldsymbol{b}$.

In other words, we would like to know that, whenever we approximate $\boldsymbol{b}$ by smooth vector fields $\boldsymbol{b}^{h}$, the classical trajectories $\boldsymbol{X}^{h}$ associated with $\boldsymbol{b}^{h}$ satisfy

$$
\lim _{h \rightarrow \infty} \boldsymbol{X}^{h}(\cdot, x)=\boldsymbol{X}(\cdot, x) \text { in } C\left([0, T] ; \mathbb{R}^{d}\right) \text { for } \mathcal{L}^{d} \text {-a.e. } x .
$$

The following simple example provides an illustration of the kind of phenomena that can occur.

EXAMPLE 1.1. Let us consider the autonomous ODE

$$
\begin{aligned}
\dot{\gamma}(t) & =\sqrt{|\gamma(t)|}, \\
\gamma(0) & =x_{0} .
\end{aligned}
$$

Then, solutions of the ODE are not unique for $x_{0}=-c^{2}<0$. Indeed, they reach the origin in a time $2 c$, where they can stay for an arbitrary time $T$, then continue as $x(t)=\frac{1}{4}(t-T-2 c)^{2}$. Let us consider, for instance, the Lipschitz approximation (that could easily be made smooth) of $b(\gamma)=\sqrt{|\gamma|}$ by

$$
b_{\varepsilon}(\gamma):= \begin{cases}\sqrt{|\gamma|} & \text { if }-\infty<\gamma \leqslant-\varepsilon^{2}, \\ \varepsilon & \text { if }-\varepsilon^{2} \leqslant \gamma \leqslant \lambda_{\varepsilon}-\varepsilon^{2}, \\ \sqrt{\gamma-\lambda_{\varepsilon}+2 \varepsilon^{2}} & \text { if } \lambda_{\varepsilon}-\varepsilon^{2} \leqslant \gamma<+\infty,\end{cases}
$$

with $\lambda_{\varepsilon}-\varepsilon^{2}>0$. Solutions of the approximating ODE starting from $-c^{2}$ reach the value $-\varepsilon^{2}$ in time $t_{\varepsilon}=2(c-\varepsilon)$, and continue with constant speed $\varepsilon$ until they reach $\lambda_{\varepsilon}-\varepsilon^{2}$ in time $T_{\varepsilon}=\lambda_{\varepsilon} / \varepsilon$. They then continue as $\lambda_{\varepsilon}-2 \varepsilon^{2}+\frac{1}{4}\left(t-t_{\varepsilon}-T_{\varepsilon}\right)^{2}$.

Choosing $\lambda_{\varepsilon}=\varepsilon T$ with $T>0$, by this approximation we select the solutions that, when at the origin, do not move exactly for a time $T$.

Other approximations, for instance $b_{\varepsilon}(\gamma)=\sqrt{\varepsilon+|\gamma|}$, select the solutions that move immediately away from the singularity at $\gamma=0$. Among all possibilities, this family of solutions $x\left(t, x_{0}\right)$ is singled out by the property that $x(t, \cdot)_{\#} \mathcal{L}^{1}$ is absolutely continuous with respect to $\mathcal{L}^{1}$, so no concentration of trajectories occurs at the origin ${ }^{1}$. To see this fact, notice that we can integrate in time the identity

$$
0=x(t, \cdot)_{\#} \mathcal{L}^{1}(\{0\})=\mathcal{L}^{1}\left(\left\{x_{0}: x\left(t, x_{0}\right)=0\right\}\right)
$$

\footnotetext{
${ }^{1}$ In this paper we are using the notation $f_{\#} \mu$ for the push-forward operator between measures; see $(2.2)$ and (2.3).
} 
and use Fubini's theorem to obtain

$$
0=\int \mathcal{L}^{1}\left(\left\{t: x\left(t, x_{0}\right)=0\right\}\right) \mathrm{d} x_{0} .
$$

Hence, for $\mathcal{L}^{1}$-a.e. $x_{0}, x\left(\cdot, x_{0}\right)$ does not stay at 0 for a strictly positive set of times.

We will see that there is a close link between (PDE) and (ODE), first investigated in a non-smooth setting by DiPerna and Lions [72].

Let us now make some basic technical remarks regarding the continuity equation and the transport equation.

REMARK 1.2 (regularity in space of $\boldsymbol{b}_{t}$ and $\mu_{t}$ ).

(1) Since the continuity equation (PDE) is in divergence form, it makes sense without any regularity requirement on $\boldsymbol{b}_{t}$ and/or $\mu_{t}$, provided that

$$
\int_{I} \int_{A}\left|\boldsymbol{b}_{t}\right| \mathrm{d}\left|\mu_{t}\right| \mathrm{d} t<+\infty \quad \forall A \Subset \mathbb{R}^{d}
$$

(here and in the following, $A \Subset B$ means that $\bar{A}$ is a compact subset of $B$ ). However, when we consider possibly singular measures $\mu_{t}$, we must address the fact that the product $\boldsymbol{b}_{t} \mu_{t}$ is sensitive to modifications of $\boldsymbol{b}_{t}$ in $\mathcal{L}^{d}$-negligible sets. In the Sobolev or BV case we will consider only measures $\mu_{t}=w_{t} \mathcal{L}^{d}$, so everything is well posed.

(2) On the other hand, due to the fact that the distribution $\boldsymbol{b}_{t} \cdot \nabla w$ is defined by

$\left\langle\boldsymbol{b}_{t} \cdot \nabla w, \varphi\right\rangle:=-\int_{I} \int w\left\langle\boldsymbol{b}_{t}, \nabla \varphi\right\rangle \mathrm{d} x \mathrm{~d} t-\int_{I}\left\langle\mathrm{D}_{x} \cdot \boldsymbol{b}_{t}, w_{t} \varphi_{t}\right\rangle \mathrm{d} t, \quad \varphi \in C_{\mathrm{c}}^{\infty}\left(I \times \mathbb{R}^{d}\right)$

(a definition consistent with the case in which $w_{t}$ is smooth), the transport equation makes sense only if we assume that $\mathrm{D}_{x} \cdot \boldsymbol{b}_{t}=\operatorname{div} \boldsymbol{b}_{t} \mathcal{L}^{d}$ for $\mathcal{L}^{1}$-a.e. $t \in I$. See also $[38,40]$ for more refined results on the transport equation when $\boldsymbol{b}$ satisfies a one-sided Lipschitz condition.

We next consider the problem of the time continuity of $t \mapsto \mu_{t}$ and $t \mapsto w_{t}$.

REMARK 1.3 (regularity in time of $\mu_{t}$ ). For any test function $\varphi \in C_{\mathrm{c}}^{\infty}\left(\mathbb{R}^{d}\right)$, condition (1.1) gives

$$
\frac{\mathrm{d}}{\mathrm{d} t} \int_{\mathbb{R}^{d}} \varphi \mathrm{d} \mu_{t}=\int_{\mathbb{R}^{d}} \boldsymbol{b}_{t} \cdot \nabla \varphi \mathrm{d} \mu_{t} \in L^{1}(I),
$$

and therefore the map $t \mapsto\left\langle\mu_{t}, \varphi\right\rangle$, for given $\varphi$, has a unique uniformly continuous representative in $I$. By a simple density argument, we can find a unique representative $\tilde{\mu}_{t}$ independent of $\varphi$, such that $t \mapsto\left\langle\tilde{\mu}_{t}, \varphi\right\rangle$ is uniformly continuous in $I$ for any $\varphi \in C_{\mathrm{c}}^{\infty}\left(\mathbb{R}^{d}\right)$. We will always work with this representative, so that $\mu_{t}$ will be well defined for all $t$ and even at the endpoints of $I$.

An analogous remark applies for solutions of the transport equation.

There are some other important links between the two equations.

(1) The transport equation reduces to the continuity equation for the case in which $c_{t}=-w_{t} \operatorname{div} \boldsymbol{b}_{t}$. 
(2) Formally, one can establish a duality between the two equations via the (formal) identity

$$
\begin{aligned}
\frac{\mathrm{d}}{\mathrm{d} t} \int w_{t} \mathrm{~d} \mu_{t} & =\int \frac{\mathrm{d}}{\mathrm{d} t} w_{t} \mathrm{~d} \mu_{t}+\int \frac{\mathrm{d}}{\mathrm{d} t} \mu_{t} w_{t} \\
& =\int\left(-\boldsymbol{b}_{t} \cdot \nabla w_{t}+c\right) \mathrm{d} \mu_{t}+\int \boldsymbol{b}_{t} \cdot \nabla w_{t} \mathrm{~d} \mu_{t} \\
& =\int c \mathrm{~d} \mu_{t} .
\end{aligned}
$$

This duality method is a classical tool to prove uniqueness in a sufficiently smooth setting (but see also $[38,40]$ ).

(3) Finally, if we denote by $\boldsymbol{Y}(t, s, x)$ the solution of the ODE at time $t$, starting from $x$ at the initial time $s$, i.e.

$$
\frac{\mathrm{d}}{\mathrm{d} t} \boldsymbol{Y}(t, s, x)=\boldsymbol{b}_{t}(\boldsymbol{Y}(t, s, x)), \quad \boldsymbol{Y}(s, s, x)=x,
$$

then $\boldsymbol{Y}(t, \cdot, \cdot)$ are themselves solutions of the transport equation. To see this, it suffices to differentiate the semigroup identity

$$
\boldsymbol{Y}(t, s, \boldsymbol{Y}(s, l, x))=\boldsymbol{Y}(t, l, x)
$$

w.r.t. $s$ to obtain, after the change of variables $y=\boldsymbol{Y}(s, l, x)$, the equation

$$
\frac{\mathrm{d}}{\mathrm{d} s} \boldsymbol{Y}(t, s, y)+\boldsymbol{b}_{s}(y) \cdot \nabla \boldsymbol{Y}(t, s, y)=0 .
$$

This property is used in an essential way in [72] to characterize the flow $\boldsymbol{Y}$ and to prove its stability properties. The approach developed here, based on [9], is based on a careful analysis of the measures transported by the flow, and ultimately on the homogeneous continuity equation only.

The layout of this paper is as follows. In $\S 2$ we review the Cauchy-Lipschitz theory and we derive classical representation formulae for the solution of the continuity and the transport equations in the Lipschitz regularity setting. Section 3 is devoted to general principles, somewhat extending the classical theory of characteristics to the non-smooth setting, relating the uniqueness of the ODE to the uniqueness of the PDE. The main tool here is the superposition principle, asserting that every positive measure solution to the continuity equation can indeed be realized as a suitable 'probabilistic push-forward' of the initial datum along a (possibly multi-valued) ODE flow. We then introduce the notion of Lagrangian flow (the suitable notion of solution to the ODE in the non-smooth framework) and prove that the well-posedness of the PDE with velocity field $\boldsymbol{b}$ implies the well-posedness of the Lagrangian flow associated with $b$.

We then start the study of the PDE in various weak regularity contexts. In $\S 4$ we introduce the notion of a renormalized solution, prove the well-posedness of the PDE for Sobolev vector fields, and describe the consequences for the Lagrangian flow; this is extended to vector fields with bounded variation in $\S 5$. 
In the subsequent two sections we describe an alternative approach to the theory of Lagrangian flows, based on suitable quantitative estimates, without reference to the PDE theory. This is first carried out for Sobolev $W^{1, p}$ vector fields with $p>1$ in $\S 6$, by showing how suitable bounds on an integral quantity measuring a logarithmic distance between Lagrangian flows imply well-posedness, quantified stability and mild regularity for the Lagrangian flow, and then extended in $\S 7$ to $W^{1,1}$ vector fields and to vector fields whose derivative is given by a singular integral of an $L^{1}$ function.

In $\S 8$ we summarize two applications to nonlinear PDEs (the Keyfitz-Kranzer system of conservation laws and the semi-geostrophic equations) and in $\S 9$ we collect some open problems and comment on further literature on the subject.

\section{The transport equation and the continuity equation within the Cauchy-Lipschitz framework}

In this section we recall the classical representation formulae for solutions of the continuity or transport equation for the case in which

$$
\boldsymbol{b} \in L^{1}\left([0, T] ; W^{1, \infty}\left(\mathbb{R}^{d} ; \mathbb{R}^{d}\right)\right) .
$$

Under this assumption it is well known that solutions $\boldsymbol{X}(t, \cdot)$ of the ODE are unique and stable. Quantitative information can be obtained by differentiation:

$$
\begin{aligned}
\frac{\mathrm{d}}{\mathrm{d} t}|\boldsymbol{X}(t, x)-\boldsymbol{X}(t, y)|^{2} & =2\left\langle\boldsymbol{b}_{t}(\boldsymbol{X}(t, x))-\boldsymbol{b}_{t}(\boldsymbol{X}(t, y)), \boldsymbol{X}(t, x)-\boldsymbol{X}(t, y)\right\rangle \\
& \leqslant 2 \operatorname{Lip}\left(\boldsymbol{b}_{t}\right)|\boldsymbol{X}(t, x)-\boldsymbol{X}(t, y)|^{2}
\end{aligned}
$$

(here $\operatorname{Lip}(f)$ denotes the smallest Lipschitz constant of $f$ ), so that Gronwall's lemma immediately gives

$$
\operatorname{Lip}(\boldsymbol{X}(t, \cdot)) \leqslant \exp \left(\int_{0}^{t} \operatorname{Lip}\left(\boldsymbol{b}_{s}\right) \mathrm{d} s\right) .
$$

Turning to the continuity equation, uniqueness of measure-valued solutions can be proved by the duality method. Or, following the techniques developed in this paper, it can be proved in a more general setting for positive measure-valued solutions (via the superposition principle) and for signed solutions $\mu_{t}=w_{t} \mathcal{L}^{d}$ (via the theory of renormalized solutions). So, in this section we focus only on the existence and the representation issues.

Given metric spaces $X$ and $Y$ and a Borel map $f: X \rightarrow Y$, we shall use in this paper the notation $f_{\#}$ for the push-forward operator mapping non-negative Borel measures in $X$ to non-negative Borel measures in $Y$, namely,

$$
f_{\#} \mu(B):=\mu\left(f^{-1}(B)\right) \quad B \subset Y \text { Borel. }
$$

Notice that $f_{\#}$ is mass preserving, so that it maps finite Borel measures to finite Borel measures and probability measures to probability measures. Note also the basic change of variables formula

$$
\int_{Y} \phi \mathrm{d} f_{\#} \mu=\int_{X} \phi \circ f \mathrm{~d} \mu
$$

for $\phi: Y \rightarrow[0,+\infty]$ Borel, that we will frequently and tacitly use in the following. 
The representation formula for solutions to the continuity equation is indeed very simple.

Proposition 2.1. For any probability measure $\bar{\mu}$ in $\mathbb{R}^{d}$, the solution of the continuity equation is given by

$$
\mu_{t}:=\boldsymbol{X}(t, \cdot)_{\#} \bar{\mu}, \quad \text { i.e. } \int_{\mathbb{R}^{d}} \varphi \mathrm{d} \mu_{t}=\int_{\mathbb{R}^{d}} \varphi(\boldsymbol{X}(t, x)) \mathrm{d} \bar{\mu}(x) .
$$

Proof. Notice first that we need only check the distributional identity $\mathrm{d} \mu_{t} / \mathrm{d} t+\mathrm{D}_{x}$. $\left(\boldsymbol{b}_{t} \mu_{t}\right)=0$ on test functions of the form $\psi(t) \varphi(x)$, so that

$$
\int_{\mathbb{R}} \psi^{\prime}(t)\left\langle\mu_{t}, \varphi\right\rangle \mathrm{d} t+\int_{\mathbb{R}} \psi(t) \int_{\mathbb{R}^{d}}\left\langle\boldsymbol{b}_{t}, \nabla \varphi\right\rangle \mathrm{d} \mu_{t} \mathrm{~d} t=0 .
$$

This means that we have to check that $t \mapsto\left\langle\mu_{t}, \varphi\right\rangle$ belongs to $W^{1,1}(0, T)$ for any $\varphi \in C_{\mathrm{c}}^{\infty}\left(\mathbb{R}^{d}\right)$ and that its distributional derivative is $\int_{\mathbb{R}^{d}}\left\langle\boldsymbol{b}_{t}, \nabla \varphi\right\rangle \mathrm{d} \mu_{t}$.

We show first that this map is absolutely continuous, and particularly that it is $W^{1,1}(0, T)$; then one needs only to compute the pointwise derivative. For every choice of finitely many, say $n$, pairwise disjoint intervals $\left(a_{i}, b_{i}\right) \subset[0, T]$ we have

$$
\begin{aligned}
\sum_{i=1}^{n}\left|\varphi\left(\boldsymbol{X}\left(b_{i}, x\right)\right)-\varphi\left(\boldsymbol{X}\left(a_{i}, x\right)\right)\right| & \leqslant\|\nabla \varphi\|_{\infty} \int_{\bigcup_{i}\left(a_{i}, b_{i}\right)}|\dot{\boldsymbol{X}}(t, x)| \mathrm{d} t \\
& \leqslant\|\nabla \varphi\|_{\infty} \int_{\bigcup_{i}\left(a_{i}, b_{i}\right)} \sup \left|\boldsymbol{b}_{t}\right| \mathrm{d} t
\end{aligned}
$$

and therefore an integration with respect to $\bar{\mu}$ gives

$$
\sum_{i=1}^{n}\left|\left\langle\mu_{b_{i}}-\mu_{a_{i}}, \varphi\right\rangle\right| \leqslant\|\nabla \varphi\|_{\infty} \int_{\bigcup_{i}\left(a_{i}, b_{i}\right)} \sup \left|\boldsymbol{b}_{t}\right| \mathrm{d} t .
$$

The absolute continuity of the integral shows that the right-hand side can be made small when $\sum_{i}\left(b_{i}-a_{i}\right)$ is small. This proves the absolute continuity. For any $x$ the identity $\dot{\boldsymbol{X}}(t, x)=\boldsymbol{b}_{t}(\boldsymbol{X}(t, x))$ is fulfilled for $\mathcal{L}^{1}$-a.e. $t \in[0, T]$. By Fubini's theorem, we then also know that, for $\mathcal{L}^{1}$-a.e. $t \in[0, T]$, the previous identity holds for $\bar{\mu}$-a.e. $x$, and therefore dominated convergence gives

$$
\begin{aligned}
\frac{\mathrm{d}}{\mathrm{d} t}\left\langle\mu_{t}, \varphi\right\rangle & =\frac{\mathrm{d}}{\mathrm{d} t} \int_{\mathbb{R}^{d}} \varphi(\boldsymbol{X}(t, x)) \mathrm{d} \bar{\mu}(x) \\
& =\int_{\mathbb{R}^{d}}\left\langle\nabla \varphi(\boldsymbol{X}(t, x)), \boldsymbol{b}_{t}(\boldsymbol{X}(t, x))\right\rangle \mathrm{d} \bar{\mu}(x) \\
& =\left\langle\boldsymbol{b}_{t} \mu_{t}, \nabla \varphi\right\rangle
\end{aligned}
$$

for $\mathcal{L}^{1}$-a.e. $t \in[0, T]$.

For the case in which $\bar{\mu}=\rho \mathcal{L}^{d}$ we can say something more, proving that the measures $\mu_{t}=\boldsymbol{X}(t, \cdot)_{\#} \bar{\mu}$ are absolutely continuous w.r.t. $\mathcal{L}^{d}$ and explicitly computing their density. Let us start by recalling the classical area formula: if $f: \mathbb{R}^{d} \rightarrow \mathbb{R}^{d}$ is a (locally) Lipschitz map, then

$$
\int_{A} g|J f| \mathrm{d} x=\int_{\mathbb{R}^{d}} \sum_{x \in A \cap f^{-1}(y)} g(x) \mathrm{d} y
$$


for any Borel set $A \subset \mathbb{R}^{d}$, where $J f=\operatorname{det} \nabla f$ (recall that, by Rademacher's theorem, Lipschitz functions are differentiable $\mathcal{L}^{d}$-almost everywhere). Assuming in addition that $f$ is $1-1$ and onto and that $|J f|>0 \mathcal{L}^{d}$-almost everywhere on $A$, we can set $A=f^{-1}(B)$ and $g=\rho /|J f|$ to obtain

$$
\int_{f^{-1}(B)} \rho \mathrm{d} x=\int_{B} \frac{\rho}{|J f|} \circ f^{-1} \mathrm{~d} y .
$$

In other words, we have a formula for the push-forward:

$$
f_{\#}\left(\rho \mathcal{L}^{d}\right)=\frac{\rho}{|J f|} \circ f^{-1} \mathcal{L}^{d}
$$

In our case $f(x)=\boldsymbol{X}(t, x)$ is surely $1-1$, onto and Lipschitz. It remains to show that $|J \boldsymbol{X}(t, \cdot)|$ does not vanish: in fact, one can show that $J \boldsymbol{X}>0$ and

$$
\exp \left[-\int_{0}^{t}\left\|\left[\operatorname{div} \boldsymbol{b}_{s}\right]^{-}\right\|_{\infty} \mathrm{d} s\right] \leqslant J \boldsymbol{X}(t, x) \leqslant \exp \left[\int_{0}^{t}\left\|\left[\operatorname{div} \boldsymbol{b}_{s}\right]^{+}\right\|_{\infty} \mathrm{d} s\right]
$$

for $\mathcal{L}^{d}$-a.e. $x$, thanks to the following fact, whose proof is left as an exercise.

EXERCISE 2.2. If $\boldsymbol{b}$ is smooth, we have

$$
\frac{\mathrm{d}}{\mathrm{d} t} J \boldsymbol{X}(t, x)=\operatorname{div} \boldsymbol{b}_{t}(\boldsymbol{X}(t, x)) J \boldsymbol{X}(t, x) .
$$

Hint: use the ODE $\mathrm{d} \nabla \boldsymbol{X} / \mathrm{d} t=\nabla \boldsymbol{b}_{t}(\boldsymbol{X}) \nabla \boldsymbol{X}$.

The previous exercise gives that, in the smooth case, $J \boldsymbol{X}(\cdot, x)$ solves a linear ODE with the initial condition $J \boldsymbol{X}(0, x)=1$, whence the estimates on $J \boldsymbol{X}$ follow. In the general case the upper estimate on $J \boldsymbol{X}$ still holds by a smoothing argument, thanks to the lower semi-continuity of

$$
\Phi(v):= \begin{cases}\|J v\|_{\infty} & \text { if } J v \geqslant 0 \mathcal{L}^{d} \text {-almost everywhere } \\ +\infty & \text { otherwise }\end{cases}
$$

with respect to the $w^{*}$-topology of $W^{1, \infty}\left(\mathbb{R}^{d} ; \mathbb{R}^{d}\right)$ (see $[74, \S 8.2 .4]$ ). This is indeed the supremum of the family of $\Phi_{p}^{1 / p}$, where $\Phi_{p}$ are the polyconvex (and therefore lower semi-continuous (l.s.c.)) functionals

$$
\Phi_{p}(v):=\int_{B_{p}}|\chi(J v)|^{p} \mathrm{~d} x .
$$

Here $\chi(t)$ is equal to $\infty$ on $(-\infty, 0)$ and equal to $t$ on $[0,+\infty)$, is l.s.c. and convex. The lower estimate can be obtained by applying the upper one in a time-reversed situation.

We now turn to the representation of solutions of the transport equation.

Proposition 2.3. If $w \in L_{\mathrm{loc}}^{1}\left([0, T] \times \mathbb{R}^{d}\right)$ solves

$$
\frac{\mathrm{d}}{\mathrm{d} t} w_{t}+\boldsymbol{b} \cdot \nabla w=c \in L_{\mathrm{loc}}^{1}\left([0, T] \times \mathbb{R}^{d}\right)
$$


in the sense of distributions, then, for $\mathcal{L}^{d}$-a.e. $x$, we have

$$
w_{t}(\boldsymbol{X}(t, x))=w_{0}(x)+\int_{0}^{t} c_{s}(\boldsymbol{X}(s, x)) \mathrm{d} s \quad \forall t \in[0, T] .
$$

The (formal) proof is based on the simple observation that

$$
\begin{aligned}
\frac{\mathrm{d}}{\mathrm{d} t} w_{t} \circ \boldsymbol{X}(t, x) & =\frac{\mathrm{d}}{\mathrm{d} t} w_{t}(\boldsymbol{X}(t, x))+\frac{\mathrm{d}}{\mathrm{d} t} \boldsymbol{X}(t, x) \cdot \nabla w_{t}(\boldsymbol{X}(t, x)) \\
& =\frac{\mathrm{d}}{\mathrm{d} t} w_{t}(\boldsymbol{X}(t, x))+\boldsymbol{b}_{t}(\boldsymbol{X}(t, x)) \cdot \nabla w_{t}(\boldsymbol{X}(t, x)) \\
& =c_{t}(\boldsymbol{X}(t, x)) .
\end{aligned}
$$

In particular, as $\boldsymbol{X}(t, x)=\boldsymbol{Y}(t, 0, x)=[\boldsymbol{Y}(0, t, \cdot)]^{-1}(x)$, we get

$$
w_{t}(y)=w_{0}(\boldsymbol{Y}(0, t, y))+\int_{0}^{t} c_{s}(\boldsymbol{Y}(s, t, y)) \mathrm{d} s .
$$

We conclude this presentation of the classical theory by pointing out two simple local variants of the assumption $\boldsymbol{b} \in L^{1}\left([0, T] ; W^{1, \infty}\left(\mathbb{R}^{d} ; \mathbb{R}^{d}\right)\right)$ made throughout this section.

REMARK 2.4 (first local variant). The theory outlined above still works under the assumptions

$$
\boldsymbol{b} \in L^{1}\left([0, T] ; W_{\mathrm{loc}}^{1, \infty}\left(\mathbb{R}^{d} ; \mathbb{R}^{d}\right)\right), \quad \frac{|\boldsymbol{b}|}{1+|x|} \in L^{1}\left([0, T] ; L^{\infty}\left(\mathbb{R}^{d}\right)\right) .
$$

Indeed, due to the growth condition on $\boldsymbol{b}$, we still have pointwise uniqueness of the ODE and a uniform local control on the growth of $|\boldsymbol{X}(t, x)|$. Therefore, we need only consider a local Lipschitz condition w.r.t. $x$, integrable w.r.t. $t$.

The next variant will be used in the proof of the superposition principle.

REMARK 2.5 (second local variant). Still keeping the $L^{1}\left(W_{\text {loc }}^{1, \infty}\right)$ assumption, and assuming $\mu_{t} \geqslant 0$, the second growth condition on $|\boldsymbol{b}|$ can be replaced by a global, but more intrinsic, condition:

$$
\int_{0}^{T} \int_{\mathbb{R}^{d}} \frac{\left|\boldsymbol{b}_{t}\right|}{1+|x|} \mathrm{d} \mu_{t} \mathrm{~d} t<+\infty
$$

Under this assumption, one can show that, for $\bar{\mu}$-a.e. $x$, the maximal solution $\boldsymbol{X}(\cdot, x)$ of the ODE starting from $x$ is defined up to $t=T$ and the representation $\mu_{t}=$ $\boldsymbol{X}(t, \cdot)_{\#} \bar{\mu}$ still holds for $t \in[0, T]$ (see $\left.[22, \S 8.1]\right)$.

\section{ODE uniqueness versus PDE uniqueness}

In this section we illustrate some general principles, whose application may depend on specific assumptions on $\boldsymbol{b}$, relating the uniqueness of the ODE to the uniqueness of the PDE. The viewpoint adopted in this section is very close in spirit to Young's theory [106] of generalized surfaces and controls (a theory that also has 
remarkable applications to nonlinear PDEs [71,99] and calculus of variations [26]), and also has some connection with Brenier's weak solutions of incompressible Euler equations $[13,41]$, with Kantorovich's viewpoint in the theory of optimal transportation [75,95] and with Mather's theory [27,90,91]. In order to study the existence, uniqueness and stability (with respect to perturbations of the data) of solutions to the ODE, we consider suitable measures in the space of continuous maps, allowing for the superposition of trajectories. Then, in some special situations, we are able to show that this superposition actually does not occur; despite this, this 'probabilistic' interpretation is still very useful with regard to understanding the underlying techniques and to give an intrinsic characterization of the flow.

The first, very general, criterion is the following.

THEOREM 3.1. Let $A \subset \mathbb{R}^{d}$ be a Borel set. The following two properties are equivalent:

(a) solutions of the $O D E$ are unique for any $x \in A$;

(b) non-negative measure-valued solutions of the PDE are unique for any $\bar{\mu}$ concentrated in $A$, i.e. such that $\bar{\mu}\left(\mathbb{R}^{d} \backslash A\right)=0$.

Proof. It is clear that (b) implies (a) just by choosing $\bar{\mu}=\delta_{x}$ and noticing that two different solutions $\boldsymbol{X}(t), \tilde{\boldsymbol{X}}(t)$ of the ODE induce two different solutions of the PDE; namely, $\delta_{\boldsymbol{X}(t)}$ and $\delta_{\tilde{\boldsymbol{X}}(t)}$.

The converse implication is less obvious and requires the superposition principle, which we will describe below, and that provides the representation

$$
\int_{\mathbb{R}^{d}} \varphi \mathrm{d} \mu_{t}=\int_{\mathbb{R}^{d}}\left(\int_{\Gamma_{T}} \varphi(\gamma(t)) \mathrm{d} \boldsymbol{\eta}_{x}(\gamma)\right) \mathrm{d} \mu_{0}(x)
$$

with $\boldsymbol{\eta}_{x}$ probability measures concentrated on the absolutely continuous integral solutions of the ODE starting from $x$. Therefore, when these are unique, the measures $\boldsymbol{\eta}_{x}$ are unique (and are Dirac masses), and so the solutions of the PDE are unique.

We will use the shorter notation $\Gamma_{T}$ for the space $C\left([0, T] ; \mathbb{R}^{d}\right)$ and denote by $e_{t}: \Gamma_{T} \rightarrow \mathbb{R}^{d}$ the evaluation maps $\gamma \mapsto \gamma(t), t \in[0, T]$.

Definition 3.2 (superposition solutions). Let $\boldsymbol{\eta} \in \mathcal{M}_{+}\left(\mathbb{R}^{d} \times \Gamma_{T}\right)$ be a measure concentrated on the set of pairs $(x, \gamma)$ such that $\gamma$ is an absolutely continuous integral solution of the ODE with $\gamma(0)=x$. We define

$$
\left\langle\mu_{t}^{\boldsymbol{\eta}}, \varphi\right\rangle:=\int_{\mathbb{R}^{d} \times \Gamma_{T}} \varphi\left(e_{t}(\gamma)\right) \mathrm{d} \boldsymbol{\eta}(x, \gamma) \quad \forall \varphi \in C_{b}\left(\mathbb{R}^{d}\right) .
$$

By a standard approximation argument, the identity defining $\mu_{t}^{\boldsymbol{\eta}}$ holds for any Borel function $\varphi$ such that $\gamma \mapsto \varphi\left(e_{t}(\gamma)\right)$ is $\boldsymbol{\eta}$-integrable (or, equivalently, any $\mu_{t}^{\eta}$-integrable function $\varphi$ ).

Under the (local) integrability condition

$$
\int_{0}^{T} \int_{\mathbb{R}^{d} \times \Gamma_{T}} \chi_{B_{R}}\left(e_{t}\right)\left|\boldsymbol{b}_{t}\left(e_{t}\right)\right| \mathrm{d} \boldsymbol{\eta} \mathrm{d} t<+\infty \quad \forall R>0,
$$


it is not hard to see that $\mu_{t}^{\eta}$ solves the PDE with the initial condition $\bar{\mu}:=\left(\pi_{\mathbb{R}^{d}}\right)_{\#} \boldsymbol{\eta}$. Indeed, let us check first that $t \mapsto\left\langle\mu_{t}^{\eta}, \varphi\right\rangle$ is absolutely continuous for any $\varphi \in$ $C_{\mathrm{c}}^{\infty}\left(\mathbb{R}^{d}\right)$. For every choice of finitely many pairwise disjoint intervals $\left(a_{i}, b_{i}\right) \subset[0, T]$ we have

$$
\sum_{i=1}^{n}\left|\varphi\left(\gamma\left(b_{i}\right)\right)-\varphi\left(\gamma\left(a_{i}\right)\right)\right| \leqslant \operatorname{Lip}(\varphi) \int_{\bigcup_{i}\left(a_{i}, b_{i}\right)} \chi_{B_{R}}\left(\left|e_{t}(\gamma)\right|\right) \boldsymbol{b}_{t}\left(e_{t}(\gamma)\right) \mid \mathrm{d} t
$$

for $\boldsymbol{\eta}$-a.e. $(x, \gamma)$, with $R$ such that $\operatorname{supp} \varphi \subset \bar{B}_{R}$. Therefore, assuming for simplicity that $\boldsymbol{\eta}\left(\mathbb{R}^{d} \times \Gamma_{T}\right)=1$, an integration with respect to $\boldsymbol{\eta}$ gives

$$
\sum_{i=1}^{n}\left|\left\langle\mu_{b_{i}}^{\eta}, \varphi\right\rangle-\left\langle\mu_{a_{i}}^{\eta}, \varphi\right\rangle\right| \leqslant \operatorname{Lip}(\varphi) \int_{\bigcup_{i}\left(a_{i}, b_{i}\right)} \int_{\mathbb{R}^{d} \times \Gamma_{T}} \chi_{B_{R}}\left(e_{t}\right)\left|\boldsymbol{b}_{t}\left(e_{t}\right)\right| \mathrm{d} \boldsymbol{\eta} \mathrm{d} t .
$$

The absolute continuity of the integral shows that the right-hand side can be made small when $\sum_{i}\left(b_{i}-a_{i}\right)$ is small. This proves the absolute continuity.

It remains to evaluate the time derivative of $t \mapsto\left\langle\mu_{t}^{\eta}, \varphi\right\rangle$ : we know that, for $\boldsymbol{\eta}$-a.e. $(x, \gamma)$, the identity $\dot{\gamma}(t)=\boldsymbol{b}_{t}(\gamma(t))$ is fulfilled for $\mathcal{L}^{1}$-a.e. $t \in[0, T]$. Then, by Fubini's theorem, we know also that for $\mathcal{L}^{1}$-a.e. $t \in[0, T]$ the previous identity holds for $\boldsymbol{\eta}$-a.e. $(x, \gamma)$, and therefore

$$
\begin{aligned}
\frac{\mathrm{d}}{\mathrm{d} t}\left\langle\mu_{t}^{\boldsymbol{\eta}}, \varphi\right\rangle & =\frac{\mathrm{d}}{\mathrm{d} t} \int_{\mathbb{R}^{d} \times \Gamma_{T}} \varphi\left(e_{t}(\gamma)\right) \mathrm{d} \boldsymbol{\eta} \\
& =\int_{\mathbb{R}^{d} \times \Gamma_{T}}\left\langle\nabla \varphi\left(e_{t}(\gamma)\right), \boldsymbol{b}_{t}\left(e_{t}(\gamma)\right)\right\rangle \mathrm{d} \boldsymbol{\eta} \\
& =\left\langle\boldsymbol{b}_{t} \mu_{t}, \nabla \varphi\right\rangle \quad \mathcal{L}^{1} \text {-a.e. in }[0, T] .
\end{aligned}
$$

REMARK 3.3. Actually, the formula defining $\mu_{t}^{\eta}$ does not contain $x$, and so it involves only the projection of $\boldsymbol{\eta}$ on $\Gamma_{T}$. Therefore, one could also consider measures $\boldsymbol{\sigma}$ in $\Gamma_{T}$, concentrated on the set of solutions of the ODE (for an arbitrary initial point $x$ ). These two viewpoints are basically equivalent: given $\boldsymbol{\eta}$ one can build $\boldsymbol{\sigma}$ just by projection onto $\Gamma_{T}$; given $\boldsymbol{\sigma}$, one can consider the conditional probability measures $\boldsymbol{\eta}_{x}$ concentrated on the solutions of the ODE starting from $x$ induced by the random variable $\gamma \mapsto \gamma(0)$ in $\Gamma_{T}$, the law $\bar{\mu}$ (i.e. the push forward) of the same random variable and recover $\boldsymbol{\eta}$ via

$$
\int_{\mathbb{R}^{d} \times \Gamma_{T}} \varphi(x, \gamma) \mathrm{d} \boldsymbol{\eta}(x, \gamma):=\int_{\mathbb{R}^{d}}\left(\int_{\Gamma_{T}} \varphi(x, \gamma) \mathrm{d} \boldsymbol{\eta}_{x}(\gamma)\right) \mathrm{d} \bar{\mu}(x) .
$$

Our viewpoint has been chosen simply for technical convenience in order to avoid the use, wherever this is possible, of the conditional probability theorem.

By restricting $\boldsymbol{\eta}$ to suitable subsets of $\mathbb{R}^{d} \times \Gamma_{T}$, several manipulations with superposition solutions of the continuity equation are possible and useful, and these are not immediate to see just at the level of general solutions of the continuity equation. This is why the following result is interesting.

Theorem 3.4 (superposition principle). Let $\mu_{t} \in \mathcal{M}_{+}\left(\mathbb{R}^{d}\right)$ solve (PDE) and assume that

$$
\int_{0}^{T} \int_{\mathbb{R}^{d}} \frac{|\boldsymbol{b}| t(x)}{1+|x|} \mathrm{d} \mu_{t}(x) \mathrm{d} t<+\infty
$$


Then $\mu_{t}$ is a superposition solution, i.e. there exists $\boldsymbol{\eta} \in \mathcal{M}_{+}\left(\mathbb{R}^{d} \times \Gamma_{T}\right)$ such that $\mu_{t}=\mu_{t}^{\eta}$ for any $t \in[0, T]$.

In the proof we use the weak convergence of positive measures, i.e. the convergence with respect to the duality with continuous and bounded functions, and the easy implication in Prokhorov compactness theorem: any tight and bounded family $\mathcal{F}$ in $\mathcal{M}_{+}(X)$ is (sequentially) relatively compact w.r.t. the weak convergence. Remember that tightness means

for any $\varepsilon>0$ there exists $K \subset X$ compact s.t. $\mu(X \backslash K)<\varepsilon \quad$ for every $\mu \in \mathcal{F}$.

A necessary and sufficient condition for tightness is the existence of a coercive functional $\Psi: X \rightarrow[0, \infty]$ such that $\int \Psi \mathrm{d} \mu \leqslant 1$ for any $\mu \in \mathcal{F}$.

Proof.

STEP 1 (smoothing [76]). We mollify $\mu_{t}$ w.r.t. the space variable with a kernel $\rho$ having finite first moment $M$ and support equal to the whole of $\mathbb{R}^{d}$ (a Gaussian, for instance), obtaining smooth and strictly positive functions $\mu_{t}^{\varepsilon}$. We also choose a function $\psi: \mathbb{R}^{d} \rightarrow[0,+\infty)$ such that $\psi(x) \rightarrow+\infty$ as $|x| \rightarrow+\infty$ and

$$
\int_{\mathbb{R}^{d}} \psi(x) \mu_{0} * \rho_{\varepsilon}(x) \mathrm{d} x \leqslant 1 \quad \forall \varepsilon \in(0,1),
$$

and a convex non-decreasing function $\Theta: \mathbb{R}^{+} \rightarrow \mathbb{R}$ having a more than linear growth at infinity such that

$$
\int_{0}^{T} \int_{\mathbb{R}^{d}} \frac{\Theta\left(\left|\boldsymbol{b}_{t}\right|(x)\right)}{1+|x|} \mathrm{d} \mu_{t} \mathrm{~d} t<+\infty
$$

(the existence of $\Theta$ is ensured by the Dunford-Pettis theorem). Defining

$$
\mu_{t}^{\varepsilon}:=\mu_{t} * \rho_{\varepsilon}, \quad \boldsymbol{b}_{t}^{\varepsilon}:=\frac{\left(\boldsymbol{b}_{t} \mu_{t}\right) * \rho_{\varepsilon}}{\mu_{t}^{\varepsilon}},
$$

it is immediate that

$$
\frac{\mathrm{d}}{\mathrm{d} t} \mu_{t}^{\varepsilon}+\mathrm{D}_{x} \cdot\left(\boldsymbol{b}_{t}^{\varepsilon} \mu_{t}^{\varepsilon}\right)=\frac{\mathrm{d}}{\mathrm{d} t} \mu_{t} * \rho_{\varepsilon}+\mathrm{D}_{x} \cdot\left(\boldsymbol{b}_{t} \mu_{t}\right) * \rho_{\varepsilon}=0
$$

and that $\boldsymbol{b}^{\varepsilon} \in L^{1}\left([0, T] ; W_{\text {loc }}^{1, \infty}\left(\mathbb{R}^{d} ; \mathbb{R}^{d}\right)\right)$. Therefore, remark 2.5 can be applied and the representation $\mu_{t}^{\varepsilon}=\boldsymbol{X}^{\varepsilon}(t, \cdot)_{\#} \mu_{0}^{\varepsilon}$ still holds. We then define

$$
\boldsymbol{\eta}^{\varepsilon}:=\left(x, \boldsymbol{X}^{\varepsilon}(\cdot, x)\right)_{\#} \mu_{0}^{\varepsilon}
$$

so that

$$
\begin{aligned}
\int_{\mathbb{R}^{d}} \varphi \mathrm{d} \mu_{t}^{\boldsymbol{\eta}_{\varepsilon}} & =\int_{\mathbb{R}^{d} \times \Gamma_{T}} \varphi(\gamma(t)) \mathrm{d} \boldsymbol{\eta}^{\varepsilon} \\
& =\int_{\mathbb{R}^{d}} \varphi\left(\boldsymbol{X}^{\varepsilon}(t, x)\right) \mathrm{d} \mu_{0}^{\varepsilon}(x) \\
& =\int_{\mathbb{R}^{d}} \varphi \mathrm{d} \mu_{t}^{\varepsilon} .
\end{aligned}
$$


SteP 2 (tightness). We will be using the inequalities

$$
((1+|x|) c) * \rho_{\varepsilon} \leqslant(1+|x|) c * \rho_{\varepsilon}+\varepsilon c * \tilde{\rho}_{\varepsilon}
$$

for $c$ a non-negative measure and $\tilde{\rho}(y)=|y| \rho(y)$, and

$$
\Theta\left(\left|\boldsymbol{b}_{t}^{\varepsilon}(x)\right|\right) \mu_{t}^{\varepsilon}(x) \leqslant\left(\Theta\left(\left|\boldsymbol{b}_{t}\right|\right) \mu_{t}\right) * \rho_{\varepsilon}(x) .
$$

The proof of the first one is elementary, while the proof of the second one follows by applying Jensen's inequality with the convex l.s.c. function $(z, t) \mapsto \Theta(|z| / t) t$ (set to $+\infty$ if $t<0$, or if $t=0$ and $z \neq 0$, and set to 0 if $z=t=0$ ) and with the measure $\rho_{\varepsilon}(x-\cdot) \mathcal{L}^{d}$.

Let us introduce the functional

$$
\Psi(x, \gamma):=\psi(x)+\int_{0}^{T} \frac{\Theta(|\dot{\gamma}|)}{1+|\gamma|} \mathrm{d} t
$$

set to $+\infty$ on $\Gamma_{T} \backslash A C\left([0, T] ; \mathbb{R}^{d}\right)$.

Using the Ascoli-Arzelà theorem, it is not hard to show that $\Psi$ is coercive (it suffices to show that $\max |\gamma|$ is bounded on the sublevels $\{\Psi \leqslant t\}$, so that $\int_{0}^{T} \Theta(|\dot{\gamma}|) \mathrm{d} t$ is also uniformly bounded on the sublevels of $\Psi$ ). Since

$$
\begin{aligned}
& \int_{\mathbb{R}^{d} \times \Gamma_{T}} \int_{0}^{T} \frac{\Theta(|\dot{\gamma}|)}{1+|\gamma|} \mathrm{d} t \mathrm{~d} \boldsymbol{\eta}^{\varepsilon}(x, \gamma)=\int_{0}^{T} \int_{\mathbb{R}^{d}} \frac{\Theta\left(\left|\boldsymbol{b}_{t}^{\varepsilon}\right|\right)}{1+|x|} \mathrm{d} \mu_{t}^{\varepsilon} \mathrm{d} t \\
& \stackrel{(3.4),(3.5)}{\leqslant}(1+\varepsilon M) \int_{0}^{T} \int_{\mathbb{R}^{d}} \frac{\Theta\left(\left|\boldsymbol{b}_{t}\right|(x)\right)}{1+|x|} \mathrm{d} \mu_{t} \mathrm{~d} t
\end{aligned}
$$

and

$$
\int_{\mathbb{R}^{d} \times \Gamma_{T}} \psi(x) \mathrm{d} \boldsymbol{\eta}^{\varepsilon}(x, \gamma)=\int_{\mathbb{R}^{d}} \psi(x) \mathrm{d} \mu_{0}^{\varepsilon} \leqslant 1,
$$

we obtain that $\int \Psi \mathrm{d} \boldsymbol{\eta}^{\varepsilon}$ is uniformly bounded for $\varepsilon \in(0,1)$, and therefore the Prokhorov compactness theorem tells us that the family $\boldsymbol{\eta}^{\varepsilon}$ is weakly sequentially relatively compact as $\varepsilon \downarrow 0$. If $\boldsymbol{\eta}$ is any limit point, we can pass to the limit in (3.3) to obtain that $\mu_{t}=\mu_{t}^{\eta}$.

STEP 3 ( $\boldsymbol{\eta}$ is concentrated on solutions of the ODE). It suffices to show that

$$
\int_{\mathbb{R}^{d} \times \Gamma_{T}} \frac{\left|\gamma(t)-x-\int_{0}^{t} \boldsymbol{b}_{s}(\gamma(s)) \mathrm{d} s\right|}{1+\max _{[0, T]}|\gamma|} \mathrm{d} \boldsymbol{\eta}=0
$$

for any $t \in[0, T]$. The technical difficulty is that this test function, due to the lack of regularity of $\boldsymbol{b}$, is not continuous. Towards this aim, we prove first that

$$
\int_{\mathbb{R}^{d} \times \Gamma_{T}} \frac{\left|\gamma(t)-x-\int_{0}^{t} \boldsymbol{c}_{s}(\gamma(s)) \mathrm{d} s\right|}{1+\max _{[0, T]}|\gamma|} \mathrm{d} \boldsymbol{\eta} \leqslant \int_{0}^{T} \int_{\mathbb{R}^{d}} \frac{\left|\boldsymbol{b}_{s}-\boldsymbol{c}_{s}\right|}{1+|x|} \mathrm{d} \mu_{s} \mathrm{~d} s
$$

for any continuous function $c$ with compact support. Then, choosing a sequence $\left(\boldsymbol{c}^{n}\right)$ converging to $\boldsymbol{b}$ in $L^{1}\left(\nu ; \mathbb{R}^{d}\right)$ with

$$
\int \varphi(s, x) \mathrm{d} \nu(s, x):=\int_{0}^{T} \int_{\mathbb{R}^{d}} \frac{\varphi(s, x)}{1+|x|} \mathrm{d} \mu_{s}(x) \mathrm{d} s,
$$


and noticing that

$$
\int_{\mathbb{R}^{d} \times \Gamma_{T}} \int_{0}^{T} \frac{\left|\boldsymbol{b}_{s}(\gamma(s))-\boldsymbol{c}_{s}^{n}(\gamma(s))\right|}{1+|\gamma(s)|} \mathrm{d} s \mathrm{~d} \boldsymbol{\eta}=\int_{0}^{T} \int_{\mathbb{R}^{d}} \frac{\left|\boldsymbol{b}_{s}-\boldsymbol{c}_{s}^{n}\right|}{1+|x|} \mathrm{d} \mu_{s} \mathrm{~d} s \rightarrow 0,
$$

we can pass to the limit in (3.7) with $\boldsymbol{c}=\boldsymbol{c}^{n}$ to obtain (3.6).

It remains to show (3.7). This is a limiting argument based on the fact that (3.6) holds for $\boldsymbol{b}^{\varepsilon}$ and $\boldsymbol{\eta}^{\varepsilon}$ :

$$
\begin{aligned}
\int_{\mathbb{R}^{d} \times \Gamma_{T}} \frac{\left|\gamma(t)-x-\int_{0}^{t} \boldsymbol{c}_{s}(\gamma(s)) \mathrm{d} s\right|}{1+\max _{[0, T]}|\gamma|} \mathrm{d} \boldsymbol{\eta}^{\varepsilon} \\
\quad=\int_{\mathbb{R}^{d}} \frac{\left|\boldsymbol{X}^{\varepsilon}(t, x)-x-\int_{0}^{t} \boldsymbol{c}_{s}\left(\boldsymbol{X}^{\varepsilon}(s, x)\right) \mathrm{d} s\right|}{1+\max _{[0, T]}\left|\boldsymbol{X}^{\varepsilon}(\cdot, x)\right|} \mathrm{d} \mu_{0}^{\varepsilon}(x) \\
\quad=\int_{\mathbb{R}^{d}} \frac{\left|\int_{0}^{t} \boldsymbol{b}_{s}^{\varepsilon}\left(\boldsymbol{X}^{\varepsilon}(s, x)\right)-\boldsymbol{c}_{s}\left(\boldsymbol{X}^{\varepsilon}(s, x)\right) \mathrm{d} s\right|}{1+\max _{[0, T]}\left|\boldsymbol{X}^{\varepsilon}(\cdot, x)\right|} \mathrm{d} \mu_{0}^{\varepsilon}(x) \\
\quad \leqslant \int_{0}^{t} \int_{\mathbb{R}^{d}} \frac{\left|\boldsymbol{b}_{s}^{\varepsilon}-\boldsymbol{c}_{s}\right|}{1+|x|} \mathrm{d} \mu_{s}^{\varepsilon} \mathrm{d} s \\
\quad \leqslant \int_{0}^{t} \int_{\mathbb{R}^{d}} \frac{\left|\boldsymbol{b}_{s}^{\varepsilon}-\boldsymbol{c}_{s}^{\varepsilon}\right|}{1+|x|} \mathrm{d} \mu_{s}^{\varepsilon} \mathrm{d} s+\int_{0}^{t} \int_{\mathbb{R}^{d}} \frac{\left|\boldsymbol{c}_{s}^{\varepsilon}-\boldsymbol{c}_{s}\right|}{1+|x|} \mathrm{d} \mu_{s}^{\varepsilon} \mathrm{d} s \\
\quad \leqslant \int_{0}^{t} \int_{\mathbb{R}^{d}} \frac{\left|\boldsymbol{b}_{s}-\boldsymbol{c}_{s}\right|}{1+|x|} \mathrm{d} \mu_{s} \mathrm{~d} s+\int_{0}^{t} \int_{\mathbb{R}^{d}} \frac{\left|\boldsymbol{c}_{s}^{\varepsilon}-\boldsymbol{c}_{s}\right|}{1+|x|} \mathrm{d} \mu_{s}^{\varepsilon} \mathrm{d} s .
\end{aligned}
$$

In the last inequalities we added and subtracted $\boldsymbol{c}_{t}^{\varepsilon}:=\left(\boldsymbol{c}_{t} \mu_{t}\right) * \rho_{\varepsilon} / \mu_{t}^{\varepsilon}$. Since $\boldsymbol{c}_{t}^{\varepsilon} \rightarrow \boldsymbol{c}_{t}$ uniformly as $\varepsilon \downarrow 0$ thanks to the uniform continuity of $\boldsymbol{c}$, passing to the limit in the chain of inequalities above we obtain (3.7).

The applicability of theorem 3.1 is strongly limited by the fact that, on the one hand, pointwise uniqueness properties for the ODE are known only in very special situations, for instance, when there is a Lipschitz or a one-sided Lipschitz (or logLipschitz, Osgood, etc.) condition on $\boldsymbol{b}$. On the other hand, uniqueness for general measure-valued solutions is also known only in special situations. It turns out that in many cases uniqueness of the PDE can only be proved in smaller classes $\mathcal{L}$ of solutions, and it is natural to think that this should be reflected in a weaker uniqueness condition at the level of the ODE.

We will see that there is indeed uniqueness in the 'selection sense'. In order to illustrate this concept, in the following we consider a convex class $\mathcal{L}_{\boldsymbol{b}}$ of measurevalued solutions $\mu_{t} \in \mathcal{M}_{+}\left(\mathbb{R}^{d}\right)$ of the continuity equation relative to $\boldsymbol{b}$, satisfying the following monotonicity property:

$$
0 \leqslant \mu_{t}^{\prime} \leqslant \mu_{t} \in \mathcal{L}_{\boldsymbol{b}} \quad \Longrightarrow \quad \mu_{t}^{\prime} \in \mathcal{L}_{\boldsymbol{b}}
$$

whenever $\mu_{t}^{\prime}$ still solves the continuity equation relative to $\boldsymbol{b}$, and satisfying the integrability condition

$$
\int_{0}^{T} \int_{\mathbb{R}^{d}} \frac{\left|\boldsymbol{b}_{t}(x)\right|}{1+|x|} \mathrm{d} \mu_{t}(x) \mathrm{d} t<+\infty .
$$


The typical application will be with absolutely continuous measures $\mu_{t}=w_{t} \mathcal{L}^{d}$ whose densities satisfy some quantitative and possibly time-dependent bound (e.g. $\left.L^{\infty}\left(L^{1}\right) \cap L^{\infty}\left(L^{\infty}\right)\right)$.

Definition $3.5\left(\mathcal{L}_{\boldsymbol{b}}\right.$-Lagrangian flows). Given the class $\mathcal{L}_{\boldsymbol{b}}$, we say that $\boldsymbol{X}(t, x)$ is an $\mathcal{L}_{\boldsymbol{b}}$-Lagrangian flow starting from $\bar{\mu} \in \mathcal{M}_{+}\left(\mathbb{R}^{d}\right)$ (at time 0 ) if the following two properties hold:

(a) $\boldsymbol{X}(\cdot, x)$ is absolutely continuous in $[0, T]$ and satisfies

$$
\boldsymbol{X}(t, x)=x+\int_{0}^{t} \boldsymbol{b}_{s}(\boldsymbol{X}(s, x)) \mathrm{d} s \quad \forall t \in[0, T]
$$

for $\bar{\mu}$-a.e. $x$;

(b) $\mu_{t}:=\boldsymbol{X}(t, \cdot)_{\#} \bar{\mu} \in \mathcal{L}_{\boldsymbol{b}}$.

Heuristically, $\mathcal{L}_{\boldsymbol{b}}$-Lagrangian flows can be thought as suitable selections of the solutions of the ODE (possibly non-unique), made in such a way as to produce a density in $\mathcal{L}_{\boldsymbol{b}}$ (see example 1.1 for an illustration of this concept).

We will show that the $\mathcal{L}_{b}$-Lagrangian flow starting from $\bar{\mu}$ is unique, modulo $\bar{\mu}$-negligible sets, whenever well-posedness for the PDE holds in the class $\mathcal{L}_{\boldsymbol{b}}$.

Before stating and proving the uniqueness theorem for $\mathcal{L}_{\boldsymbol{b}}$-Lagrangian flows, we state two elementary, but useful, results. The first one is a simple exercise.

Exercise 3.6. Let $\sigma \in \mathcal{M}_{+}\left(\Gamma_{T}\right)$ and let $D \subset[0, T]$ be a dense set. Show that $\sigma$ is a Dirac mass in $\Gamma_{T}$ if its projections $(e(t))_{\#} \sigma, t \in D$, are Dirac masses in $\mathbb{R}^{d}$.

The second one is concerned with a family of measures $\boldsymbol{\eta}_{x}$.

Lemma 3.7. Let $\boldsymbol{\eta}_{x}$ be a measurable family of positive finite measures in $\Gamma_{T}$ with the following property: for any $t \in[0, T]$ and any pair of disjoint Borel sets $E, E^{\prime} \subset \mathbb{R}^{d}$, we have

$$
\boldsymbol{\eta}_{x}(\{\gamma: \gamma(t) \in E\}) \boldsymbol{\eta}_{x}\left(\left\{\gamma: \gamma(t) \in E^{\prime}\right\}\right)=0 \quad \bar{\mu} \text {-a.e. in } \mathbb{R}^{d} .
$$

Then $\boldsymbol{\eta}_{x}$ is a Dirac mass for $\bar{\mu}$-a.e. $x$.

Proof. Taking into account exercise 3.6, for a fixed $t \in(0, T]$ it suffices to check that the measures $\lambda_{x}:=\gamma(t)_{\#} \boldsymbol{\eta}_{x}$ are Dirac masses for $\bar{\mu}$-a.e. $x$. Then (3.9) gives $\lambda_{x}(E) \lambda_{x}\left(E^{\prime}\right)=0 \bar{\mu}$-a.e. for any pair of disjoint Borel sets $E, E^{\prime} \subset \mathbb{R}^{d}$. Let $\delta>0$ and let us consider a partition of $\mathbb{R}^{d}$ in countably many Borel sets $R_{i}$ having diameter less than $\delta$. Then, as $\lambda_{x}\left(R_{i}\right) \lambda_{x}\left(R_{j}\right)=0 \mu$-a.e. whenever $i \neq j$, we have a corresponding decomposition of $\bar{\mu}$-almost all of $\mathbb{R}^{d}$ in Borel sets $A_{i}$ such that $\operatorname{supp} \lambda_{x} \subset \bar{R}_{i}$ for any $x \in A_{i}$ (just take $\left\{\lambda_{x}\left(R_{i}\right)>0\right\}$ and subtract from it all other sets $\left\{\lambda_{x}\left(R_{j}\right)>0\right\}$, $j \neq i)$. Since $\delta$ is arbitrary, the statement is proved.

TheOREM 3.8 (uniqueness of $\mathcal{L}_{\boldsymbol{b}}$-Lagrangian flows). Assume that the PDE is well posed in $\mathcal{L}_{\boldsymbol{b}}$. Then the $\mathcal{L}_{\boldsymbol{b}}$-Lagrangian flow starting from $\bar{\mu}$ is unique, i.e. two different selections $\boldsymbol{X}_{1}(t, x)$ and $\boldsymbol{X}_{2}(t, x)$ of solutions of the $O D E$ inducing solutions of the continuity equation in $\mathcal{L}_{\boldsymbol{b}}$ satisfy

$$
\boldsymbol{X}_{1}(\cdot, x)=\boldsymbol{X}_{2}(\cdot, x) \quad \text { in } \Gamma_{T} \text { for } \bar{\mu} \text {-a.e. } x .
$$


Proof. If the statement were false, we could produce a measure $\boldsymbol{\eta}$ not concentrated on a graph inducing a solution $\mu_{t}^{\eta} \in \mathcal{L}_{\boldsymbol{b}}$ of the PDE. But this is not possible, since it would contradict theorem 3.10. Such measure $\eta$ can be easily built as follows:

$$
\boldsymbol{\eta}:=\frac{1}{2}\left(\boldsymbol{\eta}^{1}+\boldsymbol{\eta}^{2}\right)=\frac{1}{2}\left[\left(x, \boldsymbol{X}_{1}(\cdot, x)\right)_{\#} \bar{\mu}+\left(x, \boldsymbol{X}_{2}(\cdot, x)\right)_{\#} \bar{\mu}\right] .
$$

Since $\mathcal{L}_{\boldsymbol{b}}$ is convex we still have $\mu_{t}^{\boldsymbol{\eta}}=\frac{1}{2}\left(\mu_{t}^{\boldsymbol{\eta}^{1}}+\mu_{t}^{\boldsymbol{\eta}^{2}}\right) \in \mathcal{L}_{\boldsymbol{b}}$.

REMARK 3.9. In the same vein, one can also show that

$$
\boldsymbol{X}_{1}(\cdot, x)=\boldsymbol{X}_{2}(\cdot, x) \quad \text { in } \Gamma_{T} \text { for } \bar{\mu}_{1} \wedge \bar{\mu}_{2} \text {-a.e. } x
$$

whenever $\boldsymbol{X}_{1}$ and $\boldsymbol{X}_{2}$ are $\mathcal{L}_{\boldsymbol{b}}$-Lagrangian flows starting, respectively, from $\bar{\mu}_{1}$ and $\bar{\mu}_{2}$.

We used the following basic result, which has some analogy with Kantorovich's and Mather's theories.

THEOREM 3.10. Assume that the PDE is well posed in $\mathcal{L}_{\boldsymbol{b}}$. Let $\boldsymbol{\eta} \in \mathcal{M}_{+}\left(\mathbb{R}^{d} \times \Gamma_{T}\right)$ be concentrated on the pairs $(x, \gamma)$ with $\gamma$ an absolutely continuous solution of the $O D E$, and assume that $\mu_{t}^{\boldsymbol{\eta}} \in \mathcal{L}_{\boldsymbol{b}}$. Then $\boldsymbol{\eta}$ is concentrated on a graph, i.e. there exists a function $x \mapsto X(\cdot, x) \in \Gamma_{T}$ such that

$$
\boldsymbol{\eta}=(x, X(\cdot, x))_{\#} \bar{\mu} \quad \text { with } \bar{\mu}:=\left(\pi_{\mathbb{R}^{d}}\right)_{\#} \boldsymbol{\eta}=\mu_{0}^{\boldsymbol{\eta}} .
$$

Proof. We use the representation (3.2) of $\boldsymbol{\eta}$ given by the disintegration theorem, the criterion stated in lemma 3.7 and argue by contradiction. If the thesis were false then $\boldsymbol{\eta}_{x}$ would not be a Dirac mass in a set of $\bar{\mu}$-positive measure and we could find $t \in(0, T]$, disjoint Borel sets $E, E^{\prime} \subset \mathbb{R}^{d}$ and a Borel set $C$ with $\bar{\mu}(C)>0$ such that

$$
\boldsymbol{\eta}_{x}(\{\gamma: \gamma(t) \in E\}) \boldsymbol{\eta}_{x}\left(\left\{\gamma: \gamma(t) \in E^{\prime}\right\}\right)>0 \quad \forall x \in C .
$$

Possibly passing to a smaller set having still strictly positive $\bar{\mu}$-measure, we can assume that

$$
0<\frac{\boldsymbol{\eta}_{x}(\{\gamma: \gamma(t) \in E\})}{\boldsymbol{\eta}_{x}\left(\left\{\gamma: \gamma(t) \in E^{\prime}\right\}\right)} \leqslant M \quad \forall x \in C
$$

for some constant $M<+\infty$. Denoting by $\beta$ the quotient in (3.10), we define measures $\boldsymbol{\eta}^{1}, \boldsymbol{\eta}^{2}$ whose disintegrations $\boldsymbol{\eta}_{x}^{1}, \boldsymbol{\eta}_{x}^{2}$ are given by

$$
\boldsymbol{\eta}_{x}^{1}:=\chi_{C}(x) \boldsymbol{\eta}_{x}\left\llcorner\{\gamma: \gamma(t) \in E\}, \quad \boldsymbol{\eta}_{x}^{2}:=\beta(x) \chi_{C}(x) \boldsymbol{\eta}_{x}\left\llcorner\left\{\gamma: \gamma(t) \in E^{\prime}\right\}\right.\right.
$$

and denote by $\mu_{t}^{i}$ the (superposition) solutions of the continuity equation induced by $\boldsymbol{\eta}^{i}$. Then

$$
\mu_{0}^{1}=\boldsymbol{\eta}_{x}(\{\gamma: \gamma(t) \in E\}) \bar{\mu}\left\llcorner C, \quad \mu_{0}^{2}=\beta(x) \boldsymbol{\eta}_{x}\left(\left\{\gamma: \gamma(t) \in E^{\prime}\right\}\right) \bar{\mu}\llcorner C\right.
$$

so that the definition of $\beta$ yields $\mu_{0}^{1}=\mu_{0}^{2}$. On the other hand, $\mu_{t}^{1}$ is orthogonal to $\mu_{t}^{2}$ : precisely, denoting by $\boldsymbol{\eta}_{t x}$ the image of $\boldsymbol{\eta}_{x}$ under the map $\gamma \mapsto \gamma(t)$, we have

$$
\mu_{t}^{1}=\int_{C} \boldsymbol{\eta}_{t x}\left\llcornerE \mathrm { d } \mu ( x ) \perp M \int _ { C } \boldsymbol { \eta } _ { t x } \left\llcorner E^{\prime} \mathrm{d} \mu(x)=\mu_{t}^{2} .\right.\right.
$$

Notice also that $\mu_{t}^{i} \leqslant \mu_{t}$ and so the monotonicity assumption (3.8) on $\mathcal{L}_{\boldsymbol{b}}$ gives $\mu_{t}^{i} \in \mathcal{L}_{\boldsymbol{b}}$. This contradicts the assumption on the validity of the comparison principle in $\mathcal{L}_{\boldsymbol{b}}$. 
Now we come to the existence of $\mathcal{L}_{b}$-Lagrangian flows.

Theorem 3.11 (existence of $\mathcal{L}_{\boldsymbol{b}}$-Lagrangian flows). Assume that the PDE is well posed in $\mathcal{L}_{\boldsymbol{b}}$ and that for some $\bar{\mu} \in \mathcal{M}_{+}\left(\mathbb{R}^{d}\right)$ there exists a solution $\mu_{t} \in \mathcal{L}_{\boldsymbol{b}}$ with $\mu_{0}=\bar{\mu}$. Then there exists a (unique) $\mathcal{L}_{\boldsymbol{b}}$-Lagrangian flow starting from $\bar{\mu}$.

Proof. By the superposition principle, we can represent $\mu_{t}$ as $\left(e_{t}\right)_{\#} \boldsymbol{\eta}$ for some $\boldsymbol{\eta} \in \mathcal{M}_{+}\left(\mathbb{R}^{d} \times \Gamma_{T}\right)$ concentrated on pairs $(x, \gamma)$ of solutions of the ODE. Then theorem 3.10 tells us that $\boldsymbol{\eta}$ is concentrated on a graph, i.e. there exists a function $x \mapsto \boldsymbol{X}(\cdot, x) \in \Gamma_{T}$ such that

$$
(x, \boldsymbol{X}(\cdot, x))_{\#} \bar{\mu}=\boldsymbol{\eta} .
$$

Pushing both sides via $e_{t}$ we obtain

$$
\boldsymbol{X}(t, \cdot)_{\#} \bar{\mu}=\left(e_{t}\right)_{\#} \boldsymbol{\eta}=\mu_{t} \in \mathcal{L}_{\boldsymbol{b}},
$$

and therefore $\boldsymbol{X}$ is a $\mathcal{L}_{\boldsymbol{b}}$-Lagrangian flow.

Finally, let us discuss the stability issue. As we will see, this is particularly relevant in connection with the applications to PDEs.

DEFINITION 3.12 (convergence of velocity fields). We define the convergence of $\boldsymbol{b}^{h}$ to $\boldsymbol{b}$ in an indirect way, defining rather a convergence of $\mathcal{L}_{\boldsymbol{b}^{h}}$ to $\mathcal{L}_{\boldsymbol{b}}$ : we require that

$$
\boldsymbol{b}^{h} \mu_{t}^{h} \rightarrow \boldsymbol{b} \mu_{t} \text { in }(0, T) \times \mathbb{R}^{d} \text { and } \mu_{t} \in \mathcal{L}_{\boldsymbol{b}}
$$

whenever $\mu_{t}^{h} \in \mathcal{L}_{\boldsymbol{b}^{h}}$ and $\mu_{t}^{h} \rightarrow \mu_{t}$ weakly for all $t \in[0, T]$.

For instance, in the typical case when $\mathcal{L}$ is bounded and closed w.r.t. the weak* topology in $L^{\infty}\left(L^{1}\right) \cap L^{\infty}\left(L^{\infty}\right)$, and

$$
\mathcal{L}_{\boldsymbol{c}}:=\mathcal{L} \cap\left\{w: \frac{\mathrm{d}}{\mathrm{d} t} w+\mathrm{D}_{x} \cdot(\boldsymbol{c} w)=0\right\},
$$

the implication is fulfilled whenever $\boldsymbol{b}^{h} \rightarrow \boldsymbol{b}$ strongly in $L_{\text {loc }}^{1}$.

For the flows, the natural convergence in the stability theorem is convergence in measure. Let us recall that a $Y$-valued sequence $\left(v_{h}\right)$ is said to converge in $\bar{\mu}$-measure to $v$ if

$$
\lim _{h \rightarrow \infty} \bar{\mu}\left(\left\{d_{Y}\left(v_{h}, v\right)>\delta\right\}\right)=0 \quad \forall \delta>0 .
$$

This is equivalent to the $L^{1}$ convergence to 0 of the $\mathbb{R}^{+}$-valued maps $1 \wedge d_{Y}\left(v_{h}, v\right)$.

Recall also that convergence $\bar{\mu}$-a.e. implies convergence in $\bar{\mu}$-measure, and that the converse implication is true passing to a suitable subsequence.

Theorem 3.13 (stability of L-Lagrangian flows). Assume that:

(i) $\mathcal{L}_{\boldsymbol{b}^{h}}$ converge to $\mathcal{L}_{\boldsymbol{b}}$;

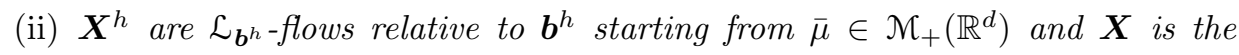
$\mathcal{L}_{\boldsymbol{b}}$-flow relative to $\boldsymbol{b}$ starting from $\bar{\mu}$; 
(iii) setting $\mu_{t}^{h}:=\boldsymbol{X}^{h}(t, \cdot)_{\#} \bar{\mu}$, we have

$$
\mu_{t}^{h} \rightarrow \mu_{t} \quad \text { weakly as } h \rightarrow \infty \text { for all } t \in[0, T]
$$

and

$$
\limsup _{h \rightarrow \infty} \int_{0}^{T} \int_{\mathbb{R}^{d}} \frac{\Theta\left(\left|\boldsymbol{b}_{t}^{h}\right|\right)}{1+|x|} \mathrm{d} \mu_{t}^{h} \mathrm{~d} t \leqslant \int_{0}^{T} \int_{\mathbb{R}^{d}} \frac{\Theta\left(\left|\boldsymbol{b}_{t}\right|\right)}{1+|x|} \mathrm{d} \mu_{t} \mathrm{~d} t<+\infty
$$

for some strictly convex function $\Theta: \mathbb{R}^{+} \rightarrow \mathbb{R}$ having more than linear growth at infinity;

(iv) the PDE is well posed in $\mathcal{L}_{\boldsymbol{b}}$.

Then $\mu_{t}=\boldsymbol{X}(t, \cdot)_{\#} \bar{\mu}$ and $x \mapsto \boldsymbol{X}^{h}(\cdot, x)$ converge to $x \mapsto \boldsymbol{X}(\cdot, x)$ in $\bar{\mu}$-measure, i.e.

$$
\lim _{h \rightarrow \infty} \int_{\mathbb{R}^{d}} 1 \wedge \sup _{[0, T]}\left|\boldsymbol{X}^{h}(\cdot, x)-\boldsymbol{X}(\cdot, x)\right| \mathrm{d} \bar{\mu}(x)=0 .
$$

Proof. Following the same strategy used in the proof of the superposition principle, we push $\bar{\mu}$ onto the graph of the map $x \mapsto \boldsymbol{X}^{h}(\cdot, x)$, i.e.

$$
\boldsymbol{\eta}^{h}:=\left(x, \boldsymbol{X}^{h}(\cdot, x)\right)_{\#} \bar{\mu},
$$

and we obtain, using (3.12) and the same argument used in step 2 of the proof of the superposition principle, that $\boldsymbol{\eta}^{h}$ is tight in $\mathcal{M}_{+}\left(\mathbb{R}^{d} \times \Gamma_{T}\right)$.

Now let $\boldsymbol{\eta}$ be any limit point of $\boldsymbol{\eta}^{h}$ in the weak topology. Using the same argument as in step 3 of the proof of the superposition principle, and (3.12), we obtain that $\boldsymbol{\eta}$ is concentrated on pairs $(x, \gamma)$ with $\gamma$ an absolutely continuous solution of the ODE relative to $\boldsymbol{b}$ starting from $x$. Indeed, this argument was made using only the property

$$
\lim _{h \rightarrow \infty} \int_{0}^{T} \int_{\mathbb{R}^{d}} \frac{\left|\boldsymbol{b}_{t}^{h}-\boldsymbol{c}_{t}\right|}{1+|x|} \mathrm{d} \mu_{t}^{h} \mathrm{~d} t=\int_{0}^{T} \int_{\mathbb{R}^{d}} \frac{\left|\boldsymbol{b}_{t}-\boldsymbol{c}_{t}\right|}{1+|x|} \mathrm{d} \mu_{t} \mathrm{~d} t
$$

for any continuous function $\boldsymbol{c}$ with compact support in $(0, T) \times \mathbb{R}^{d}$, and this property is ensured by lemma 3.15 below.

Let $\mu_{t}:=\left(\boldsymbol{e}_{t}\right)_{\#} \boldsymbol{\eta}$ and notice that $\mu_{t}^{h}=\left(\boldsymbol{e}_{t}\right)_{\#} \boldsymbol{\eta}^{h}$; hence $\mu_{t}^{h} \rightarrow \mu_{t}$ weakly for any $t \in[0, T]$. As $\mu_{t}^{h} \in \mathcal{L}_{\boldsymbol{b}^{h}}$, assumption (i) gives that $\mu_{t} \in \mathcal{L}_{\boldsymbol{b}}$ and assumption (iv) together with theorem 3.10 imply that $\boldsymbol{\eta}$ is concentrated on the graph of the map $x \mapsto \boldsymbol{X}(\cdot, x)$, where $\boldsymbol{X}$ is the unique $\mathcal{L}_{\boldsymbol{b}}$-Lagrangian flow. We have thus obtained that

$$
\left(x, \boldsymbol{X}^{h}(\cdot, x)\right)_{\#} \bar{\mu} \rightarrow(x, \boldsymbol{X}(\cdot, x))_{\#} \bar{\mu} .
$$

By applying the following general principle we conclude.

LEMMA 3.14 (weak convergence and convergence in measure). Let $v_{h}, v: X \rightarrow Y$ be Borel maps and let $\bar{\mu} \in \mathcal{M}_{+}(X)$. Then $v_{h} \rightarrow v$ in $\bar{\mu}$-measure if and only if

$$
\left(x, v_{h}(x)\right)_{\#} \bar{\mu} \text { converges to }(x, v(x))_{\#} \bar{\mu} \text { weakly in } \mathcal{M}_{+}(X \times Y) \text {. }
$$


Proof. If $v_{h} \rightarrow v$ in $\bar{\mu}$-measure, then $\varphi\left(x, v_{h}(x)\right)$ converges in $L^{1}(\bar{\mu})$ to $\varphi(x, v(x))$, and we immediately obtain the convergence of the push-forward measures. Conversely, let $\delta>0$ and, for any $\varepsilon>0$, let $w \in C_{b}(X ; Y)$ be such that $\bar{\mu}(\{v \neq w\}) \leqslant \varepsilon$. We define

$$
\varphi(x, y):=1 \wedge \frac{d_{Y}(y, w(x))}{\delta} \in C_{b}(X \times Y)
$$

and notice that

$$
\begin{gathered}
\bar{\mu}(\{v \neq w\})+\int_{X \times Y} \varphi \mathrm{d}\left(x, v_{h}(x)\right)_{\#} \bar{\mu} \geqslant \bar{\mu}\left(\left\{d_{Y}\left(v, v_{h}\right)>\delta\right\}\right), \\
\int_{X \times Y} \varphi \mathrm{d}(x, v(x))_{\#} \bar{\mu} \leqslant \bar{\mu}(\{w \neq v\}) .
\end{gathered}
$$

Taking into account the weak convergence of the push-forward, we obtain that

$$
\limsup _{h \rightarrow \infty} \bar{\mu}\left(\left\{d_{Y}\left(v, v_{h}\right)>\delta\right\}\right) \leqslant 2 \bar{\mu}(\{w \neq v\}) \leqslant 2 \varepsilon
$$

and, since $\varepsilon$ is arbitrary, the proof is achieved.

Lemma 3.15. Let $A \subset \mathbb{R}^{m}$ be an open set and let $\sigma^{h} \in \mathcal{M}_{+}(A)$ be weakly converging to $\sigma \in \mathcal{M}_{+}(A)$. Let $\boldsymbol{f}^{h} \in L^{1}\left(A, \sigma^{h}, \mathbb{R}^{k}\right), \boldsymbol{f} \in L^{1}\left(A, \sigma, \mathbb{R}^{k}\right)$ and assume that

(i) $\boldsymbol{f}^{h} \sigma^{h}$ weakly converge, in the duality with $C_{\mathrm{c}}\left(A ; \mathbb{R}^{k}\right)$, to $\boldsymbol{f} \sigma$,

$$
\limsup _{h \rightarrow \infty} \int_{A} \Theta\left(\left|\boldsymbol{f}^{h}\right|\right) \mathrm{d} \sigma^{h} \leqslant \int_{A} \Theta(|\boldsymbol{f}|) \mathrm{d} \sigma<+\infty
$$

for some strictly convex function $\Theta: \mathbb{R}^{+} \rightarrow \mathbb{R}$ having a more than linear growth at infinity.

Then

$$
\int_{A}\left|\boldsymbol{f}^{h}-\boldsymbol{c}\right| \mathrm{d} \sigma^{h} \rightarrow \int_{A}|\boldsymbol{f}-\boldsymbol{c}| \mathrm{d} \sigma
$$

for any $c \in C_{b}\left(A ; \mathbb{R}^{k}\right)$.

Proof. We consider the measures $\nu^{h}:=\left(x, \boldsymbol{f}^{h}(x)\right)_{\#} \sigma^{h}$ in $A \times \mathbb{R}^{k}$ and we assume, possibly extracting a subsequence, that $\nu^{h} \rightarrow \nu$, with $\nu \in \mathcal{M}_{+}\left(A \times \mathbb{R}^{k}\right)$, in the duality with $C_{\mathrm{c}}\left(A \times \mathbb{R}^{k}\right)$. Using condition (ii), the weak convergence of $\sigma^{h}$ and a truncation argument, it is easy to see that the convergence actually occurs for any continuous test function $\psi(x, y)$ satisfying

$$
\lim _{|y| \rightarrow \infty} \frac{\sup _{x}|\psi(x, y)|}{\Theta(|y|)}=0 .
$$

Furthermore, for non-negative continuous functions $\psi$, we have also

$$
\int_{A \times \mathbb{R}^{k}} \psi \mathrm{d} \nu \leqslant \liminf _{h \rightarrow \infty} \int_{A \times \mathbb{R}^{k}} \psi \mathrm{d} \nu_{h}
$$


Then, choosing test functions $\psi=\psi(x) \in C_{b}(A)$, the weak convergence of $\sigma^{h}$ to $\sigma$ gives

$$
\int_{A \times \mathbb{R}^{k}} \psi \mathrm{d} \nu=\int_{A} \psi \mathrm{d} \sigma
$$

and therefore, according to the disintegration theorem, we can represent $\nu$ as

$$
\int_{A \times \mathbb{R}^{k}} \psi(x, y) \mathrm{d} \nu(x, y)=\int_{A}\left(\int_{\mathbb{R}^{k}} \psi(x, y) \mathrm{d} \nu_{x}(y)\right) \mathrm{d} \sigma(x)
$$

for a suitable Borel family of probability measures $\nu_{x}$ in $\mathbb{R}^{k}$. Next, we can use $\psi(x) y_{j}$ as test functions and assumption (i) to obtain

$$
\lim _{h \rightarrow \infty} \int_{A} \boldsymbol{f}_{j}^{h} \psi \mathrm{d} \mu^{h}=\lim _{h \rightarrow \infty} \int_{A \times \mathbb{R}^{k}} \psi(x) y_{j} \mathrm{~d} \nu^{h}=\int_{A} \psi(x)\left(\int_{\mathbb{R}^{k}} y_{j} \mathrm{~d} \nu_{x}(y)\right) \mathrm{d} \sigma(x) .
$$

As $\psi$ and $j$ are arbitrary, this means that the first moment $\nu_{x}$, i.e. $\int y \mathrm{~d} \nu_{x}$, is equal to $\boldsymbol{f}(x)$ for $\sigma$-a.e. $x$.

On the other hand, choosing $\psi(y)=\Theta(|y|)$ as the test function in (3.13), assumption (ii) gives

$$
\begin{aligned}
\int_{A} \int_{\mathbb{R}^{k}} \Theta(|y|) \mathrm{d} \nu_{x}(y) \mathrm{d} \sigma(x) & \leqslant \liminf _{h \rightarrow \infty} \int_{A \times \mathbb{R}^{k}} \Theta(|y|) \mathrm{d} \nu^{h} \\
& =\limsup _{h \rightarrow \infty} \int_{A} \Theta\left(\left|\boldsymbol{f}^{h}\right|\right) \mathrm{d} \sigma^{h} \\
& =\int_{A} \Theta(|\boldsymbol{f}|) \mathrm{d} \sigma
\end{aligned}
$$

and hence $\int \Theta(|y|) \mathrm{d} \nu_{x}=\boldsymbol{f}(x)=\Theta\left(\left|\int y \mathrm{~d} \nu_{x}\right|\right)$ for $\sigma$-a.e. $x$. As $\Theta$ is strictly convex, this can happen only if $\nu_{x}=\delta_{\boldsymbol{f}(x)}$ for $\sigma$-a.e. $x$.

Finally, taking into account the representation (3.14) of $\nu$ with $\nu_{x}=\delta_{\boldsymbol{f}(x)}$, the convergence statement can be achieved just by choosing the test function $\psi(x, y)=$ $|y-c(x)|$.

\section{Vector fields with Sobolev spatial regularity}

Here we discuss the well-posedness of the continuity or transport equations assuming that $\boldsymbol{b}_{t}(\cdot)$ has Sobolev regularity, following [72]. The general theory previously developed then provides existence, uniqueness and stability of the L-Lagrangian flow, with $\mathcal{L}:=L^{\infty}\left(L^{1}\right) \cap L^{\infty}\left(L^{\infty}\right)$. We denote by $I \subset \mathbb{R}$ an open interval.

Definition 4.1 (renormalized solutions). Let $\boldsymbol{b} \in L_{\text {loc }}^{1}\left(I ; L_{\text {loc }}^{1}\left(\mathbb{R}^{d} ; \mathbb{R}^{d}\right)\right)$ be such that $D \cdot \boldsymbol{b}_{t}=\operatorname{div} \boldsymbol{b}_{t} \mathcal{L}^{d}$ for $\mathcal{L}^{1}$-a.e. $t \in I$, with

$$
\operatorname{div} \boldsymbol{b}_{t} \in L_{\text {loc }}^{1}\left(I ; L_{\text {loc }}^{1}\left(\mathbb{R}^{d}\right)\right) \text {. }
$$

Let $w \in L_{\mathrm{loc}}^{\infty}\left(I ; L_{\mathrm{loc}}^{\infty}\left(\mathbb{R}^{d}\right)\right)$ and assume that, in the sense of distributions, there holds

$$
c:=\frac{\mathrm{d}}{\mathrm{d} t} w+\boldsymbol{b} \cdot \nabla w \in L_{\mathrm{loc}}^{1}\left(I \times \mathbb{R}^{d}\right) .
$$


We then say that $w$ is a renormalized solution of (4.1) if

$$
\frac{\mathrm{d}}{\mathrm{d} t} \beta(w)+\boldsymbol{b} \cdot \nabla \beta(w)=c \beta^{\prime}(w) \quad \forall \beta \in C^{1}(\mathbb{R}) .
$$

Equivalently, recalling the definition of the distribution $\boldsymbol{b} \cdot \nabla w$, the definition could be given in a conservative form, writing

$$
\frac{\mathrm{d}}{\mathrm{d} t} \beta(w)+\mathrm{D}_{x} \cdot(\boldsymbol{b} \beta(w))=c \beta^{\prime}(w)+\beta(w) \operatorname{div} \boldsymbol{b}_{t} .
$$

Notice also that the concept makes sense on choosing properly the class of 'test' functions $\beta$ and also functions $w$ that do not satisfy (4.1), not even locally integrable. This is particularly relevant in connection with the DiPerna-Lions existence theorem for Boltzmann's equation [73], or with the case in which $w$ is the characteristic of an unbounded vector field $\boldsymbol{b}$.

This concept is also reminiscent of Kruzhkov's concept of an entropy solution for a scalar conservation law

$$
\frac{\mathrm{d}}{\mathrm{d} t} u+\mathrm{D}_{x} \cdot(\boldsymbol{f}(u))=0, \quad u:(0,+\infty) \times \mathbb{R}^{d} \rightarrow \mathbb{R} .
$$

In this case, only a distributional one-sided inequality is required:

$$
\frac{\mathrm{d}}{\mathrm{d} t} \eta(u)+\mathrm{D}_{x} \cdot(\boldsymbol{q}(u)) \leqslant 0
$$

for any convex entropy-entropy flux pair $(\eta, \boldsymbol{q})$ (i.e. $\eta$ is convex and $\eta^{\prime} \boldsymbol{f}^{\prime}=\boldsymbol{q}^{\prime}$ ).

REMARK 4.2 (time continuity). Using the fact that both $t \mapsto w_{t}$ and $t \mapsto \beta\left(w_{t}\right)$ have a uniformly continuous representative (w.r.t. the $w^{*}-L_{\mathrm{loc}}^{\infty}$ topology), we obtain that, for any renormalized solution $w, t \mapsto w_{t}$ has a unique representative that is continuous w.r.t. the $L_{\text {loc }}^{1}$ topology at almost every time. The proof follows by a classical weak-strong convergence argument:

$$
f_{n} \rightarrow f, \quad \beta\left(f_{n}\right) \rightarrow \beta(f) \quad \Longrightarrow \quad f_{n} \rightarrow f
$$

provided $\beta$ is strictly convex. In the case of scalar conservation laws there are analogous results $[92,103]$. We remark on the fact that, in general, a renormalized solution does not need to have a representative that is strongly continuous for every $t$. This can be seen using a variation of an example given by Depauw [70]. Depauw's example provides a divergence-free vector field $\boldsymbol{a} \in L^{\infty}\left([0,1] \times \mathbb{R}^{2} ; \mathbb{R}^{2}\right)$, with $\boldsymbol{a}(t, \cdot) \in \mathrm{BV}_{\text {loc }}\left(\mathbb{R}^{2} ; \mathbb{R}^{2}\right)$ for $\mathcal{L}^{1}$-a.e. $t \in[0,1]$ (but $\boldsymbol{a} \notin L^{1}\left([0,1] ; \mathrm{BV}_{\text {loc }}\right)$ ), such that the Cauchy problem

$$
\partial_{t} u+\boldsymbol{a} \cdot \nabla u=0, \quad u(0, \cdot)=0
$$

has a non-trivial solution, with $|\bar{u}|=1 \mathcal{L}^{3}$-almost everywhere in $[0,1] \times \mathbb{R}^{2}$ and with the property that $\bar{u}(t, \cdot) \rightarrow 0$ as $t \downarrow 0$, but this convergence is not strong. Now consider a vector field $\boldsymbol{b}$ on $[-1,1] \times \mathbb{R}^{2}$ defined as Depauw's vector field for $t>0$, and set $\boldsymbol{b}(t, x)=-\boldsymbol{a}(-t, x)$ for $t<0$. It is simple to check (as only affine functions $\tilde{\beta}(t)=a+b t$ need to be checked, because for any $\beta$ there exists an affine $\tilde{\beta}$ such 
that $\tilde{\beta}( \pm 1)=\beta( \pm 1))$ that the function

$$
\bar{w}(t, x)= \begin{cases}\bar{u}(t, x) & \text { if } t>0, \\ \bar{u}(-t, x) & \text { if } t<0,\end{cases}
$$

is a renormalized solution of $\partial_{t} w+\boldsymbol{b} \cdot \nabla w=0$, but this solution is not strongly continuous at $t=0$.

REMARK 4.3. A new insight in the theory of renormalized solutions has been obtained in [35]. In particular, it is proved that for a vector field $\boldsymbol{b} \in L^{\infty}([0, T] \times$ $\mathbb{R}^{d} ; \mathbb{R}^{d}$ ) with zero divergence (and without any regularity assumption) the following two conditions are equivalent (the $L^{2}$ framework has been considered just for simplicity):

(i) $\boldsymbol{b}$ has the uniqueness property for weak solutions in $C\left([0, T] ; w-L^{2}\left(\mathbb{R}^{d}\right)\right)$ for both the forward and the backward Cauchy problems starting, respectively, from 0 and $T$, i.e. the only solutions in $C\left([0, T] ; w-L^{2}\left(\mathbb{R}^{d}\right)\right)$ to the problems

$$
\begin{aligned}
\partial_{t} u_{F}+\boldsymbol{b} \cdot \nabla u_{F} & =0, \\
u_{F}(0, \cdot) & =0
\end{aligned}
$$

and

$$
\begin{aligned}
\partial_{t} u_{B}+\boldsymbol{b} \cdot \nabla u_{B} & =0, \\
u_{B}(T, \cdot) & =0
\end{aligned}
$$

are $u_{F} \equiv 0$ and $u_{B} \equiv 0 ;$

(ii) every weak solution in $C\left([0, T] ; w-L^{2}\left(\mathbb{R}^{d}\right)\right)$ of $\partial_{t} u+\boldsymbol{b} \cdot \nabla u=0$ is strongly continuous (i.e. lies in $\left.C\left([0, T] ; s-L^{2}\left(\mathbb{R}^{d}\right)\right)\right)$ and is a renormalized solution.

The proof of this equivalence is obtained through the study of the approximation properties of the solution of the transport equation with respect to the norm of the graph of the transport operator (see [35, theorem 2.1] for details).

Using the concept of a renormalized solution, we can prove a comparison principle (and therefore well-posedness) in the following natural class $\mathcal{L}$ :

$$
\begin{aligned}
\mathcal{L}:=\left\{w \in L^{\infty}\left([0, T] ; L^{1}\left(\mathbb{R}^{d}\right)\right) \cap L^{\infty}\left([0, T] ; L^{\infty}\left(\mathbb{R}^{d}\right)\right):\right. & \\
w & \left.\in C\left([0, T] ; w^{*}-L^{\infty}\left(\mathbb{R}^{d}\right)\right)\right\} .
\end{aligned}
$$

THEOREM 4.4 (comparison principle). Assume that

$$
\frac{|\boldsymbol{b}|}{1+|x|} \in L^{1}\left([0, T] ; L^{\infty}\left(\mathbb{R}^{d}\right)\right)+L^{1}\left([0, T] ; L^{1}\left(\mathbb{R}^{d}\right)\right)
$$

that $\mathrm{D} \cdot \boldsymbol{b}_{t}=\operatorname{div} \boldsymbol{b}_{t} \mathcal{L}^{d}$ for $\mathcal{L}^{1}$-a.e. $t \in[0, T]$ and that

$$
\left[\operatorname{div} \boldsymbol{b}_{t}\right]^{-} \in L_{\mathrm{loc}}^{1}\left([0, T) \times \mathbb{R}^{d}\right) .
$$

Setting $\boldsymbol{b}_{t} \equiv 0$ for $t<0$, assume in addition that any solution of $(4.1)$ in $(-\infty, T) \times$ $\mathbb{R}^{d}$ is renormalized. Then the comparison principle and, therefore, uniqueness for the continuity equation both hold in the class $\mathcal{L}$ defined in (4.2). 
Proof. By the linearity of the equation, it suffices to show that $w \in \mathcal{L}$ and $w_{0} \leqslant 0$ implies $w_{t} \leqslant 0$ for any $t \in[0, T]$. We extend first the PDE to negative times, setting $w_{t}=w_{0}$. Then, fix a cut-off function $\varphi \in C_{\mathrm{c}}^{\infty}\left(\mathbb{R}^{d}\right)$ with $\operatorname{supp} \varphi \subset \bar{B}_{2}(0)$ and $\varphi \equiv 1$ on $B_{1}(0)$, and the renormalization functions

$$
\beta_{\varepsilon}(t):=\sqrt{\varepsilon^{2}+\left(t^{+}\right)^{2}}-\varepsilon \in C^{1}(\mathbb{R}) .
$$

Notice that

$$
\beta_{\varepsilon}(t) \uparrow t^{+} \quad \text { as } \varepsilon \downarrow 0, \quad t \beta_{\varepsilon}^{\prime}(t)-\beta_{\varepsilon}(t) \in[0, \varepsilon] .
$$

We know that

$$
\frac{\mathrm{d}}{\mathrm{d} t} \beta_{\varepsilon}\left(w_{t}\right)+\mathrm{D}_{x} \cdot\left(\boldsymbol{b} \beta_{\varepsilon}\left(w_{t}\right)\right)=\operatorname{div} \boldsymbol{b}_{t}\left(\beta_{\varepsilon}\left(w_{t}\right)-w_{t} \beta_{\varepsilon}^{\prime}\left(w_{t}\right)\right)
$$

in the sense of distributions in $(-\infty, T) \times \mathbb{R}^{d}$. Plugging $\varphi_{R}(\cdot):=\varphi(\cdot / R)$, with $R \geqslant 1$, into the PDE, we obtain

$$
\begin{aligned}
& \frac{\mathrm{d}}{\mathrm{d} t} \int_{\mathbb{R}^{d}} \varphi_{R} \beta_{\varepsilon}\left(w_{t}\right) \mathrm{d} x \\
&=\int_{\mathbb{R}^{d}} \beta_{\varepsilon}\left(w_{t}\right)\left\langle\boldsymbol{b}_{t}, \nabla \varphi_{R}\right\rangle \mathrm{d} x+\int_{\mathbb{R}^{d}} \varphi_{R} \operatorname{div} \boldsymbol{b}_{t}\left(\beta_{\varepsilon}\left(w_{t}\right)-w_{t} \beta_{\varepsilon}^{\prime}\left(w_{t}\right)\right) \mathrm{d} x .
\end{aligned}
$$

Splitting $\boldsymbol{b}$ as $\boldsymbol{b}_{1}+\boldsymbol{b}_{2}$, with

$$
\frac{\boldsymbol{b}_{1}}{1+|x|} \in L^{1}\left([0, T] ; L^{\infty}\left(\mathbb{R}^{d}\right)\right) \quad \text { and } \quad \frac{\boldsymbol{b}_{2}}{1+|x|} \in L^{1}\left([0, T] ; L^{1}\left(\mathbb{R}^{d}\right)\right),
$$

and using the inequality

$$
\frac{1}{R} \chi_{\{R \leqslant|x| \leqslant 2 R\}} \leqslant \frac{3}{1+|x|} \chi_{\{R \leqslant|x|\}},
$$

we can estimate the first integral in the right-hand side with

$$
3\|\nabla \varphi\|_{\infty}\left\|\frac{\boldsymbol{b}_{1 t}}{1+|x|}\right\|_{\infty} \int_{\{|x| \geqslant R\}}\left|w_{t}\right| \mathrm{d} x+3\|\nabla \varphi\|_{\infty}\left\|w_{t}\right\|_{\infty} \int_{\{|x| \geqslant R\}} \frac{\left|\boldsymbol{b}_{1 t}\right|}{1+|x|} \mathrm{d} x .
$$

The second integral can be estimated with

$$
\varepsilon \int_{\mathbb{R}^{d}} \varphi_{R}\left[\operatorname{div} \boldsymbol{b}_{t}\right]^{-} \mathrm{d} x .
$$

Passing to the limit first as $\varepsilon \downarrow 0$ and then as $R \rightarrow+\infty$ and using the integrability assumptions on $b$ and $w$, we get

$$
\frac{\mathrm{d}}{\mathrm{d} t} \int_{\mathbb{R}^{d}} w_{t}^{+} \mathrm{d} x \leqslant 0
$$

in the distribution sense in $\mathbb{R}$. Since the function vanishes for negative times, this suffices to conclude using Gronwall's lemma.

REMARK 4.5. It would be nice to have a completely nonlinear comparison principle between renormalized solutions, as in the Kruzhkov theory. Here, on the other hand, we rather used the fact that the difference of the two solutions is renormalized. 
In any case, DiPerna and Lions proved that all distributional solutions are renormalized when there is Sobolev regularity with respect to the spatial variables.

TheOREM 4.6. Let $b \in L_{\mathrm{loc}}^{1}\left(I ; W_{\mathrm{loc}}^{1,1}\left(\mathbb{R}^{d} ; \mathbb{R}^{d}\right)\right)$ and let $w \in L_{\mathrm{loc}}^{\infty}\left(I \times \mathbb{R}^{d}\right)$ be a distributional solution of (4.1). Then $w$ is a renormalized solution.

Proof. We mollify the PDE with respect to the spatial variables and we set

$$
r^{\varepsilon}:=(\boldsymbol{b} \cdot \nabla w) * \rho_{\varepsilon}-\boldsymbol{b} \cdot\left(\nabla\left(w * \rho_{\varepsilon}\right)\right), \quad w^{\varepsilon}:=w * \rho_{\varepsilon}
$$

to obtain

$$
\frac{\mathrm{d}}{\mathrm{d} t} w^{\varepsilon}+\boldsymbol{b} \cdot \nabla w^{\varepsilon}=c * \rho_{\varepsilon}-r^{\varepsilon}
$$

By the smoothness of $w^{\varepsilon}$ w.r.t. $x$, the PDE above tells us that $\mathrm{d} w_{t}^{\varepsilon} / \mathrm{d} t \in L_{\text {loc }}^{1}$, and therefore $w^{\varepsilon} \in W_{\text {loc }}^{1,1}\left(I \times \mathbb{R}^{d}\right)$ and we can apply the standard chain rule in Sobolev spaces, getting

$$
\frac{\mathrm{d}}{\mathrm{d} t} \beta\left(w^{\varepsilon}\right)+\boldsymbol{b} \cdot \nabla \beta\left(w^{\varepsilon}\right)=\beta^{\prime}\left(w^{\varepsilon}\right) c * \rho_{\varepsilon}-\beta^{\prime}\left(w^{\varepsilon}\right) r^{\varepsilon} .
$$

When we let $\varepsilon \downarrow 0$, the convergence in the distribution sense of all terms in the identity above is trivial, with the exception of the last one. To ensure its convergence to 0 , it seems necessary to show that $r^{\varepsilon} \rightarrow 0$ strongly in $L_{\text {loc }}^{1}$ (remember that $\beta^{\prime}\left(w^{\varepsilon}\right)$ is locally equibounded w.r.t. $\varepsilon$ ). It will be proven in the next proposition that this is indeed the case, and it is exactly here that the Sobolev regularity plays a role.

Proposition 4.7 (strong convergence of commutators). If $w \in L_{\mathrm{loc}}^{\infty}\left(I \times \mathbb{R}^{d}\right)$ and $\boldsymbol{b} \in L_{\mathrm{loc}}^{1}\left(I ; W_{\mathrm{loc}}^{1,1}\left(\mathbb{R}^{d} ; \mathbb{R}^{d}\right)\right)$, we have

$$
L_{\mathrm{loc}}^{1}-\lim _{\varepsilon \downarrow 0}(\boldsymbol{b} \cdot \nabla w) * \rho_{\varepsilon}-\boldsymbol{b} \cdot\left(\nabla\left(w * \rho_{\varepsilon}\right)\right)=0 .
$$

Proof. Playing with the definitions of $\boldsymbol{b} \cdot \nabla w$ and the convolution product of a distribution and a smooth function, one first proves the identity

$$
r^{\varepsilon}(t, x)=\int_{\mathbb{R}^{d}} w(t, z)\left(\boldsymbol{b}_{t}(z)-\boldsymbol{b}_{t}(x)\right) \cdot \nabla \rho_{\varepsilon}(x-z) \mathrm{d} z-\left(w \operatorname{div} \boldsymbol{b}_{t}\right) * \rho_{\varepsilon}(x) .
$$

Indeed, to prove (4.6) we introduce the commutators in the (easier) conservative form

$$
R^{\varepsilon}:=\left(\mathrm{D}_{x} \cdot(\boldsymbol{b} w)\right) * \rho_{\varepsilon}-\mathrm{D}_{x} \cdot\left(\boldsymbol{b} w^{\varepsilon}\right)
$$

(here we again set $w^{\varepsilon}:=w * \rho_{\varepsilon}$ ). In order to get (4.6) it suffices to show that $R^{\varepsilon}=L^{\varepsilon}-w^{\varepsilon} \operatorname{div} \boldsymbol{b}_{t}$, where

$$
L^{\varepsilon}(t, x):=\int_{\mathbb{R}^{d}} w(t, z)\left(\boldsymbol{b}_{t}(z)-\boldsymbol{b}_{t}(x)\right) \cdot \nabla \rho_{\varepsilon}(x-z) \mathrm{d} z
$$


Indeed, for any test function $\varphi$, we have that $\left\langle R^{\varepsilon}, \varphi\right\rangle$ is given by

$$
\begin{aligned}
-\int_{I} \int w \boldsymbol{b} \cdot \nabla \rho_{\varepsilon} * \varphi \mathrm{d} y \mathrm{~d} t-\int_{I} \int \varphi \boldsymbol{b} \cdot \nabla \rho_{\varepsilon} * w \mathrm{~d} x \mathrm{~d} t-\int_{I} \int w^{\varepsilon} \varphi \operatorname{div} \boldsymbol{b}_{t} \mathrm{~d} t \\
=-\int_{I} \iint w_{t}(y) \boldsymbol{b}_{t}(y) \cdot \nabla \rho_{\varepsilon}(y-x) \varphi(x) \mathrm{d} x \mathrm{~d} y \mathrm{~d} t \\
\quad-\int_{I} \iint \boldsymbol{b}_{t}(x) \nabla \rho_{\varepsilon}(x-y) w_{t}(y) \varphi(x) \mathrm{d} y \mathrm{~d} x \mathrm{~d} t-\int_{I} \int w^{\varepsilon} \varphi \operatorname{div} \boldsymbol{b}_{t} \mathrm{~d} x \mathrm{~d} t \\
=\int_{I} \int L^{\varepsilon} \varphi \mathrm{d} x \mathrm{~d} t-\int_{I} \int w^{\varepsilon} \varphi \operatorname{div} \boldsymbol{b}_{t} \mathrm{~d} x \mathrm{~d} t
\end{aligned}
$$

(in the last equality we used the fact that $\nabla \rho$ is odd).

Now we change variables and write (4.6) in the form

$$
r^{\varepsilon}(t, x)=\int_{\mathbb{R}^{d}} w(t, x-\varepsilon y) \frac{\left(\boldsymbol{b}_{t}(x-\varepsilon y)-\boldsymbol{b}_{t}(x)\right) \cdot \nabla \rho(y)}{\varepsilon} \mathrm{d} y-\left(w \operatorname{div} \boldsymbol{b}_{t}\right) * \rho_{\varepsilon}(x) .
$$

Then one uses the strong convergence of translations in $L^{p}$ and the strong convergence of the difference quotients (a property that characterizes functions in Sobolev spaces)

$$
\frac{u(x+\varepsilon z)-u(x)}{\varepsilon} \rightarrow \nabla u(x) z \quad \text { strongly in } L_{\mathrm{loc}}^{1} \text { for } u \in W_{\mathrm{loc}}^{1,1}
$$

to obtain that $r^{\varepsilon}$ strongly converges in $L_{\text {loc }}^{1}\left(I \times \mathbb{R}^{d}\right)$ to

$$
-w(t, x) \int_{\mathbb{R}^{d}}\left\langle\nabla \boldsymbol{b}_{t}(x) y, \nabla \rho(y)\right\rangle \mathrm{d} y-w(t, x) \operatorname{div} \boldsymbol{b}_{t}(x) .
$$

The elementary identity

$$
\int_{\mathbb{R}^{d}} y_{i} \frac{\partial \rho}{\partial y_{j}}(y) \mathrm{d} y=-\delta_{i j}
$$

then shows that the limit is 0 (this can also be derived by the fact that, in any case, the limit of $r^{\varepsilon}$ in the distribution sense should be 0 , so the main point here is the strength of the convergence).

In this context, given $\bar{\mu}=\rho \mathcal{L}^{d}$ with $\rho \in L^{1} \cap L^{\infty}$, the $\mathcal{L}$-Lagrangian flow starting from $\bar{\mu}$ (at time 0 ) is defined by the following two properties:

(a) $\boldsymbol{X}(\cdot, x)$ is absolutely continuous in $[0, T]$ and satisfies

$$
\boldsymbol{X}(t, x)=x+\int_{0}^{t} \boldsymbol{b}_{s}(\boldsymbol{X}(s, x)) \mathrm{d} s \quad \forall t \in[0, T] \text { for } \bar{\mu} \text {-a.e. } x ;
$$

(b) $\boldsymbol{X}(t, \cdot)_{\#} \bar{\mu} \leqslant C \mathcal{L}^{d}$ for all $t \in[0, T]$ with $C$ independent of $t$.

Assumption (b) expresses in a quantitative way the condition that 'trajectories do not concentrate too much'. It can equivalently be stated as

$$
\int_{\mathbb{R}^{d}} \varphi(\boldsymbol{X}(t, x)) \mathrm{d} x \leqslant C \int_{\mathbb{R}^{d}} \varphi(y) \mathrm{d} y
$$

for all positive $\varphi \in C_{\mathrm{c}}\left(\mathbb{R}^{d}\right)$. 
Summing up what we have obtained so far, the general theory provides us with the following existence and uniqueness result.

Theorem 4.8 (existence and uniqueness of $\mathcal{L}$-Lagrangian flows). Let

$$
\boldsymbol{b} \in L^{1}\left([0, T] ; W_{\text {loc }}^{1,1}\left(\mathbb{R}^{d} ; \mathbb{R}^{d}\right)\right)
$$

satisfy

(i) $|\boldsymbol{b}| /(1+|x|) \in L^{1}\left([0, T] ; L^{1}\left(\mathbb{R}^{d}\right)\right)+L^{1}\left([0, T] ; L^{\infty}\left(\mathbb{R}^{d}\right)\right)$;

(ii) $\left[\operatorname{div} \boldsymbol{b}_{t}\right]^{-} \in L^{1}\left([0, T] ; L^{\infty}\left(\mathbb{R}^{d}\right)\right)$.

Then the $\mathcal{L}$-Lagrangian flow relative to $\boldsymbol{b}$ exists and is unique.

Proof. By the previous results, well-posedness holds for the continuity equation relative to $\boldsymbol{b}$. The general theory previously developed therefore applies and theorem 3.8 provides uniqueness of the $\mathcal{L}$-Lagrangian flow.

As for the existence, the general theory (theorem 3.11) still tells us that it can be achieved provided that we are able to solve, within $\mathcal{L}$, the continuity equation

$$
\frac{\mathrm{d}}{\mathrm{d} t} w+\mathrm{D}_{x} \cdot(\boldsymbol{b} w)=0
$$

for any non-negative initial datum $w_{0} \in L^{1} \cap L^{\infty}$. The existence of these solutions can be immediately achieved by a smoothing argument: we approximate $\boldsymbol{b}$ in $L_{\text {loc }}^{1}$ by smooth $\boldsymbol{b}^{h}$ with a uniform bound in $L^{1}\left(L^{\infty}\right)$ for $\left[\operatorname{div} \boldsymbol{b}_{t}^{h}\right]^{-}$. This bound, in turn, provides a uniform lower bound on $J \boldsymbol{X}^{h}$ and finally a uniform upper bound on $w_{t}^{h}=\left(w_{0} / J \boldsymbol{X}_{t}^{h}\right) \circ\left(\boldsymbol{X}_{t}^{h}\right)^{-1}$, solving

$$
\frac{\mathrm{d}}{\mathrm{d} t} w^{h}+\mathrm{D}_{x} \cdot\left(\boldsymbol{b}^{h} w^{h}\right)=0 .
$$

Therefore, any weak limit of $w^{h}$ solves (4.8).

Notice also that, choosing for instance a Gaussian, we obtain that the $\mathcal{L}$-Lagrangian flow is well defined up to $\mathcal{L}^{d}$-negligible sets (and is independent of $\bar{\mu} \ll \mathcal{L}^{d}$, thanks to remark 3.9).

It is interesting to compare our characterization of Lagrangian flows with the one given in [72]. Heuristically, while the DiPerna-Lions one is based on the semigroup of transformations $x \mapsto \boldsymbol{X}(t, x)$, ours is based on the properties of the map $x \mapsto$ $\boldsymbol{X}(\cdot, x)$.

REMARK 4.9. The definition of the flow in [72] is based on the following three properties:

(a)

$$
\frac{\partial \boldsymbol{Y}}{\partial t}(t, s, x)=b(t, \boldsymbol{Y}(t, s, x)) \quad \text { and } \quad \boldsymbol{Y}(s, s, x)=x
$$

in the distribution sense in $(0, T) \times \mathbb{R}^{d}$;

(b) the image $\lambda_{t}$ of $\mathcal{L}^{d}$ under $\boldsymbol{Y}(t, s, \cdot)$ satisfies

$$
\frac{1}{C} \mathcal{L}^{d} \leqslant \lambda_{t} \leqslant C \mathcal{L}^{d} \quad \text { for some constant } C>0 ;
$$


(c) for all $s, s^{\prime}, t \in[0, T]$ we have

$$
\boldsymbol{Y}\left(t, s, \boldsymbol{Y}\left(s, s^{\prime}, x\right)\right)=\boldsymbol{Y}\left(t, s^{\prime}, x\right) \text { for } \mathcal{L}^{d} \text {-a.e. } x \text {. }
$$

Then, $\boldsymbol{Y}(t, s, x)$ corresponds, in our notation, to the flow $\boldsymbol{X}^{s}(t, x)$ starting at time $s$ (well defined, even for $t<s$, if one has two-sided $L^{\infty}$ bounds on the divergence).

In our setting, condition (c) can be recovered as a consequence of the following argument: to fix the ideas assume that $s^{\prime} \leqslant s \leqslant T$ and define

$$
\tilde{\boldsymbol{X}}(t, x):= \begin{cases}\boldsymbol{X}^{s^{\prime}}(t, x) & \text { if } t \in\left[s^{\prime}, s\right], \\ \boldsymbol{X}^{s}\left(t, \boldsymbol{X}^{s^{\prime}}(s, x)\right) & \text { if } t \in[s, T] .\end{cases}
$$

It can immediately be seen that $\tilde{\boldsymbol{X}}(\cdot, x)$ is an integral solution of the ODE in $\left[s^{\prime}, T\right]$ for $\mathcal{L}^{d}$-a.e. $x$ and that $\tilde{\boldsymbol{X}}(t, \cdot)_{\#} \bar{\mu}$ is bounded by $C^{2} \mathcal{L}^{d}$. Theorem 4.8 (with $s^{\prime}$ as initial time) then gives $\tilde{\boldsymbol{X}}(\cdot, x)=\boldsymbol{X}\left(\cdot, s^{\prime}, x\right)$ in $\left[s^{\prime}, T\right]$ for $\mathcal{L}^{d}$-a.e. $x$, whence (c) follows.

Moreover, the stability theorem 3.13 can be read in this context as follows. For simplicity, we state it only in the case of equibounded vector fields (see [11] for more general results).

Theorem 4.10 (stability). Let $\boldsymbol{b}^{h}, \boldsymbol{b} \in L^{1}\left([0, T] ; W_{\text {loc }}^{1,1}\left(\mathbb{R}^{d} ; \mathbb{R}^{d}\right)\right)$, let $\boldsymbol{X}^{h}$ and $\boldsymbol{X}$ be the $\mathcal{L}$-Lagrangian flows relative to $\boldsymbol{b}^{h}$ and $\boldsymbol{b}$, respectively, let $\bar{\mu}=\rho \mathcal{L}^{d} \in \mathcal{M}_{+}\left(\mathbb{R}^{d}\right)$ and assume that

(i) $\boldsymbol{b}^{h} \rightarrow \boldsymbol{b}$ in $L_{\text {loc }}^{1}\left((0, T) \times \mathbb{R}^{d}\right)$,

(ii) $\left|\boldsymbol{b}_{h}\right| \leqslant C$ for some constant $C$ independent of $h$,

(iii) $\left[\operatorname{div} \boldsymbol{b}_{t}^{h}\right]^{-}$is bounded in $L^{1}\left([0, T] ; L^{\infty}\left(\mathbb{R}^{d}\right)\right)$.

Then,

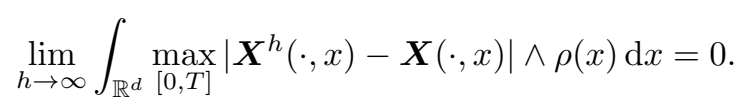

Proof. It is not restrictive, by an approximation argument, to assume that $\rho$ has compact support. Under this assumption, (i) and (iii) ensure that $\mu_{t}^{h} \leqslant M \chi_{B_{R}} \mathcal{L}^{d}$ for some constants $M$ and $R$ independent of $h$ and $t$. Denoting by $\mu_{t}$ the weak limit of $\mu_{t}^{h}$ and choosing $\Theta(z)=|z|^{2}$ in theorem 3.13(iii), we have to check that

$$
\lim _{h \rightarrow \infty} \int_{0}^{T} \int_{\mathbb{R}^{d}} \frac{\left|\boldsymbol{b}_{h}\right|^{2}}{1+|x|} \mathrm{d} \mu_{t}^{h} \mathrm{~d} t=\int_{0}^{T} \int_{\mathbb{R}^{d}} \frac{|\boldsymbol{b}|^{2}}{1+|x|} \mathrm{d} \mu_{t} \mathrm{~d} t .
$$

Let $\varepsilon>0$ and let $B \subset(0, T) \times B_{R}$ be an open set given by Egorov's theorem such that $\boldsymbol{b}_{h} \rightarrow \boldsymbol{b}$ uniformly on $[0, T] \times B_{R} \backslash B$ and $\mathcal{L}^{d+1}(B)<\varepsilon$. Also, let $\tilde{\boldsymbol{b}}_{\varepsilon}$ be such that $\left|\tilde{\boldsymbol{b}}_{\varepsilon}\right| \leqslant C$ and $\tilde{\boldsymbol{b}}_{\varepsilon}=\boldsymbol{b}$ on $[0, T] \times B_{R} \backslash B$. We write

$$
\begin{aligned}
\int_{0}^{T} \int_{\mathbb{R}^{d}} \frac{\left|\boldsymbol{b}_{h}\right|^{2}}{1+|x|} \mathrm{d} \mu_{t}^{h} \mathrm{~d} t-\int_{0}^{T} \int_{\mathbb{R}^{d}} \frac{\left|\tilde{\boldsymbol{b}}_{\varepsilon}\right|^{2}}{1+|x|} \mathrm{d} \mu_{t}^{h} \mathrm{~d} t \\
=\int_{[0, T] \times B_{R} \backslash B} \frac{\left|\boldsymbol{b}_{h}\right|^{2}-\left|\tilde{\boldsymbol{b}}_{\varepsilon}\right|^{2}}{1+|x|} \mathrm{d} \mu_{t}^{h} \mathrm{~d} t+\int_{B} \frac{\left|\boldsymbol{b}_{h}\right|^{2}-|\tilde{\boldsymbol{b}}|^{2}}{1+|x|} \mathrm{d} \mu_{t}^{h} \mathrm{~d} t,
\end{aligned}
$$


so that

$$
\limsup _{h \rightarrow \infty}\left|\int_{0}^{T} \int_{\mathbb{R}^{d}} \frac{\left|\boldsymbol{b}_{h}\right|^{2}}{1+|x|} \mathrm{d} \mu_{t}^{h} \mathrm{~d} t-\int_{0}^{T} \int_{\mathbb{R}^{d}} \frac{\left|\tilde{\boldsymbol{b}}_{\varepsilon}\right|^{2}}{1+|x|} \mathrm{d} \mu_{t} \mathrm{~d} t\right| \leqslant 2 C^{2} M \varepsilon .
$$

Since $\varepsilon$ is arbitrary and

$$
\lim _{\varepsilon \rightarrow 0} \int_{0}^{T} \int_{\mathbb{R}^{d}} \frac{\left|\tilde{\boldsymbol{b}}_{\varepsilon}\right|^{2}}{1+|x|} \mathrm{d} \mu_{t} \mathrm{~d} t=\int_{0}^{T} \int_{\mathbb{R}^{d}} \frac{|\boldsymbol{b}|^{2}}{1+|x|} \mathrm{d} \mu_{t} \mathrm{~d} t
$$

this proves that (4.9) is fulfilled.

Finally, we conclude this section with an illustration of some recent results $[15$, 19,83 ] that seem to be more specific to the Sobolev case, concerned with the 'differentiability' w.r.t. $x$ of the flow $\boldsymbol{X}(t, x)$ (see [11] for a more detailed treatment of this topic). These results provide a sort of bridge to the standard Cauchy-Lipschitz calculus.

THEOREM 4.11. There exist Borel maps $L_{t}: \mathbb{R}^{d} \rightarrow M^{d \times d}$ satisfying

$$
\lim _{h \rightarrow 0} \frac{\boldsymbol{X}(t, x+h)-\boldsymbol{X}(t, x)-L_{t}(x) h}{|h|}=0 \quad \text { locally in measure }
$$

for any $t \in[0, T]$. If, in addition, we assume that

$$
\int_{0}^{T} \int_{B_{R}}\left|\nabla \boldsymbol{b}_{t}\right| \ln \left(2+\left|\nabla \boldsymbol{b}_{t}\right|\right) \mathrm{d} x \mathrm{~d} t<+\infty \quad \forall R>0
$$

then the flow has the following 'local' Lipschitz property: for any $\varepsilon>0$ there exists a Borel set $A$ with $\bar{\mu}\left(\mathbb{R}^{d} \backslash A\right)<\varepsilon$ such that $\left.\boldsymbol{X}(t, \cdot)\right|_{A}$ is Lipschitz for any $t \in[0, T]$.

According to this result, $L$ can be thought of as a (very) weak derivative of the flow $\boldsymbol{X}$. It is still not clear whether the local Lipschitz property holds in the $W_{\text {loc }}^{1,1}$ case, or in the $\mathrm{BV}_{\text {loc }}$ case discussed in the next section. Also compare this result with the approximate differentiability results presented in $\S 6.5$.

\section{Vector fields with BV spatial regularity}

In this section we prove the renormalization theorem 4.6 under the weaker assumption of a BV dependence w.r.t. the spatial variables, but still assuming that

$$
D \cdot \boldsymbol{b}_{t} \ll \mathcal{L}^{d} \text { for } \mathcal{L}^{1} \text {-a.e. } t \in(0, T) \text {. }
$$

TheOREM 5.1. Let $\boldsymbol{b} \in L_{\text {loc }}^{1}\left((0, T) ; \mathrm{BV}_{\text {loc }}\left(\mathbb{R}^{d} ; \mathbb{R}^{d}\right)\right)$ satisfy (5.1). Any distributional solution $w \in L_{\mathrm{loc}}^{\infty}\left((0, T) \times \mathbb{R}^{d}\right)$ of

$$
\frac{\mathrm{d}}{\mathrm{d} t} w+\mathrm{D}_{x} \cdot(\boldsymbol{b} w)=c \in L_{\mathrm{loc}}^{1}\left((0, T) \times \mathbb{R}^{d}\right)
$$

is then a renormalized solution.

We try to give a reasonably detailed proof of this result, referring to the original paper [9] for minor details. Before doing that we set up some notation, denoting 
by $D \boldsymbol{b}_{t}=D^{a} \boldsymbol{b}_{t}+D^{s} \boldsymbol{b}_{t}=\nabla \boldsymbol{b}_{t} \mathcal{L}^{d}+D^{s} \boldsymbol{b}_{t}$ the Radon-Nikodým decomposition of $D \boldsymbol{b}_{t}$ in absolutely continuous and singular part w.r.t. $\mathcal{L}^{d}$. We also introduce the space-time measures $|D \boldsymbol{b}|$ and $\left|D^{s} \boldsymbol{b}\right|$ by integration w.r.t. the time variable, i.e.

$$
\begin{gathered}
\int \varphi(t, x) \mathrm{d}|D \boldsymbol{b}|:=\int_{0}^{T} \int_{\mathbb{R}^{d}} \varphi(t, x) \mathrm{d}\left|D \boldsymbol{b}_{t}\right| \mathrm{d} t, \\
\int \varphi(t, x) \mathrm{d}\left|D^{s} \boldsymbol{b}\right|:=\int_{0}^{T} \int_{\mathbb{R}^{d}} \varphi(t, x) \mathrm{d}\left|D^{s} \boldsymbol{b}_{t}\right| \mathrm{d} t .
\end{gathered}
$$

We shall also assume, by homogeneity and locality of the arguments involved, that $\|w\|_{\infty} \leqslant 1$.

Proof. We shall find two estimates on the commutators, quite sensitive to the choice of the convolution kernel, and then combine them in a (pointwise) kernel optimization argument.

STEP 1 (anisotropic estimate). Let us start from the expression

$$
r^{\varepsilon}(t, x)=\int_{\mathbb{R}^{d}} w(t, x-\varepsilon y) \frac{\left(\boldsymbol{b}_{t}(x-\varepsilon y)-\boldsymbol{b}_{t}(x)\right) \cdot \nabla \rho(y)}{\varepsilon} \mathrm{d} y-\left(w \operatorname{div} \boldsymbol{b}_{t}\right) * \rho_{\varepsilon}(x)
$$

of the commutators $(\boldsymbol{b} \cdot \nabla w) * \rho_{\varepsilon}-\boldsymbol{b} \cdot\left(\nabla\left(w * \rho_{\varepsilon}\right)\right)$ : since $\boldsymbol{b}_{t} \notin W^{1,1}$ we can no longer use the strong convergence of the difference quotients. However, for any function $u \in \mathrm{BV}_{\text {loc }}$ and any $z \in \mathbb{R}^{d}$ with $|z|<\varepsilon$, we have a classical $L^{1}$ estimate on the difference quotients

$$
\int_{K}|u(x+z)-u(x)| \mathrm{d} x \leqslant\left|\mathrm{D}_{z} u\right|\left(K_{\varepsilon}\right) \quad \text { for any } K \subset \mathbb{R}^{d} \text { compact, }
$$

where $\mathrm{D} u=\left(\mathrm{D}_{1} u, \ldots, \mathrm{D}_{d} u\right)$ stands for the distributional derivative of $u, \mathrm{D}_{z} u=$ $\langle\mathrm{D} u, z\rangle=\sum_{i} z_{i} \mathrm{D}_{i} u$ denotes the component along $z$ of $\mathrm{D} u$ and $K_{\varepsilon}$ is the open $\varepsilon$ neighbourhood of $K$. Its proof follows from an elementary smoothing and lower semi-continuity argument.

We notice that, setting $\mathrm{D} b_{t}=M_{t}\left|\mathrm{D} b_{t}\right|$, we have

$$
\mathrm{D}_{z}\left\langle b_{t}, \nabla \rho(z)\right\rangle=\left\langle M_{t}(\cdot) z, \nabla \rho(z)\right\rangle|\mathrm{D} b| \quad \forall z \in \mathbb{R}^{d},
$$

and therefore (using that $\operatorname{div} \boldsymbol{b}_{t} \mathcal{L}^{d}$ is the trace of $\mathrm{D}^{a} \boldsymbol{b}_{t}$ ) the $L^{1}$ estimate on difference quotients gives the anisotropic estimate

$$
\limsup _{\varepsilon \downarrow 0} \int_{K}\left|r^{\varepsilon}\right| \mathrm{d} x \leqslant \int_{K} \int_{\mathbb{R}^{d}}\left|\left\langle M_{t}(x) z, \nabla \rho(z)\right\rangle\right| \mathrm{d} z \mathrm{~d}|\mathrm{D} b|(t, x)+\mathrm{d}\left|\mathrm{D}^{a} b\right|(K)
$$

for any compact set $K \subset(0, T) \times \mathbb{R}^{d}$.

STEP 2 (isotropic estimate). On the other hand, a different estimate of the commutators, which reduces to the standard one when $b(t, \cdot) \in W_{\text {loc }}^{1,1}$, can be achieved as follows. Let us start from the case $d=1$ : if $\mu$ is an $\mathbb{R}^{m}$-valued measure in $\mathbb{R}$ with locally finite variation, then by Jensen's inequality the functions

$$
\hat{\mu}_{\varepsilon}(t):=\frac{\mu([t, t+\varepsilon])}{\varepsilon}=\mu * \frac{\chi_{[-\varepsilon, 0]}}{\varepsilon}(t), \quad t \in \mathbb{R},
$$


satisfy

$$
\int_{K}\left|\hat{\mu}_{\varepsilon}\right| \mathrm{d} t \leqslant|\mu|\left(K_{\varepsilon}\right) \text { for any compact set } K \subset \mathbb{R}
$$

where $K_{\varepsilon}$ is again the open $\varepsilon$ neighbourhood of $K$. A density argument based on $(5.4)$ then shows that $\hat{\mu}_{\varepsilon}$ converges in $L_{\text {loc }}^{1}(\mathbb{R})$ to the density of $\mu$ with respect to $\mathcal{L}^{1}$ whenever $\mu \ll \mathcal{L}^{1}$. If $u \in \mathrm{BV}_{\text {loc }}$ and $\varepsilon>0$, we know that

$$
\frac{u(x+\varepsilon)-u(x)}{\varepsilon}=\frac{\mathrm{D} u([x, x+\varepsilon])}{\varepsilon}=\frac{\mathrm{D}^{a} u([x, x+\varepsilon])}{\varepsilon}+\frac{\mathrm{D}^{s} u([x, x+\varepsilon])}{\varepsilon}
$$

for $\mathcal{L}^{1}$-a.e. $x$ (the exceptional set possibly depends on $\varepsilon$ ). In this way we have canonically split the difference quotient of $u$ as the sum of two functions, one strongly converging to $\nabla u$ in $L_{\text {loc }}^{1}$, and the other one having an $L^{1}$-norm on any compact set $K$ asymptotically smaller than $\left|\mathrm{D}^{s} u\right|(K)$.

If we fix the direction $z$ of the difference quotient, the slicing theory of $\mathrm{BV}$ functions gives that this decomposition can also be carried on in $d$ dimensions, showing that the difference quotients

$$
\frac{\boldsymbol{b}_{t}(x+\varepsilon z)-\boldsymbol{b}_{t}(x)}{\varepsilon}
$$

can be canonically split into two parts, the first one strongly converging in $L_{\text {loc }}^{1}\left(\mathbb{R}^{d}\right)$ to $\nabla \boldsymbol{b}_{t}(x) z$ and the second one having an $L^{1}$-norm on $K$ asymptotically smaller than $\left|\left\langle\mathrm{D}^{s} \boldsymbol{b}_{t}, z\right\rangle\right|(K)$. Repeating the DiPerna-Lions argument and taking into account the error induced by the presence of the second part of the difference quotients, we then get the isotropic estimate

$$
\limsup _{\varepsilon \downarrow 0} \int_{K}\left|r^{\varepsilon}\right| \mathrm{d} x \leqslant\left(\int_{K} \int_{\mathbb{R}^{d}}|z||\nabla \rho(z)| \mathrm{d} z\right) \mathrm{d}\left|\mathrm{D}^{s} \boldsymbol{b}\right|(t, x)
$$

for any compact set $K \subset(0, T) \times \mathbb{R}^{d}$.

STEP 3 (reduction to a pointwise optimization problem). Roughly speaking, the isotropic estimate is useful in the regions where the absolutely continuous part is the dominant one, so $\left|\mathrm{D}^{s} b\right|(K) \ll\left|\mathrm{D}^{a} b\right|(K)$, while the anisotropic one turns out to be useful in the regions where the dominant part is the singular one, i.e. $\left|\mathrm{D}^{a} b\right|(K) \ll\left|\mathrm{D}^{s} b\right|(K)$. Since the two measures are mutually singular, for a typical small ball $K$ only one of these two situations occurs. Let us see how the two estimates can be combined: coming back to the smoothing scheme, we have

$$
\frac{\mathrm{d}}{\mathrm{d} t} \beta\left(w^{\varepsilon}\right)+\boldsymbol{b} \cdot \nabla \beta\left(w^{\varepsilon}\right)-\beta^{\prime}\left(w^{\varepsilon}\right) c * \rho_{\varepsilon}=\beta^{\prime}\left(w^{\varepsilon}\right) r^{\varepsilon} .
$$

Let $L$ be the supremum of $\left|\beta^{\prime}\right|$ on $[-1,1]$. Since $K$ is an arbitrary compact set, (5.5) then tells us that any limit measure $\nu$ of $\left|\beta^{\prime}\left(w^{\varepsilon}\right) r^{\varepsilon}\right| \mathcal{L}^{d}$ as $\varepsilon \downarrow 0$ satisfies

$$
\nu \leqslant L I(\rho)\left|\mathrm{D}^{s} \boldsymbol{b}\right| \quad \text { with } I(\rho):=\int_{\mathbb{R}^{d}}|z||\nabla \rho(z)| \mathrm{d} z
$$

and, in particular, is singular with respect to $\mathcal{L}^{d}$. On the other hand, the estimate (5.3) also tells us that

$$
\nu \leqslant L \int_{\mathbb{R}^{d}}|\langle M .(\cdot) z, \nabla \rho(z)\rangle| \mathrm{d} z|\mathrm{D} \boldsymbol{b}|+\mathrm{d}\left|\mathrm{D}^{a} b\right|(K) .
$$


The second estimate and the singularity of $\nu$ with respect to $\mathcal{L}^{d}$ give

$$
\nu \leqslant L \int_{\mathbb{R}^{d}}|\langle M \cdot(\cdot) z, \nabla \rho(z)\rangle| \mathrm{d} z\left|\mathrm{D}^{s} \boldsymbol{b}\right|
$$

Notice that in this way we got rid of the potentially dangerous term $I(\rho)$ : in fact, we shall choose very anisotropic kernels $\rho$ on which $I(\rho)$ can be arbitrarily large. The measure $\nu$ can of course depend on the choice of $\rho$, but (5.6) tells us that the 'defect' measure

$$
\sigma:=\frac{\mathrm{d}}{\mathrm{d} t} \beta\left(w_{t}\right)+\boldsymbol{b} \cdot \nabla \beta\left(w_{t}\right)-c_{t} \beta^{\prime}\left(w_{t}\right),
$$

clearly independent of $\rho$, satisfies $|\sigma| \leqslant \nu$. Eventually we obtain

$$
|\sigma| \leqslant L \Lambda(M .(\cdot), \rho)\left|\mathrm{D}^{s} \boldsymbol{b}\right| \quad \text { with } \Lambda(N, \rho):=\int_{\mathbb{R}^{d}}|\langle N z, \nabla \rho(z)\rangle| \mathrm{d} z .
$$

For $(x, t)$ fixed, we are thus led to the minimum problem

$$
G(N):=\inf \left\{\Lambda(N, \rho): \rho \in C_{\mathrm{c}}^{\infty}\left(B_{1}\right), \rho \geqslant 0, \int_{\mathbb{R}^{d}} \rho=1\right\}
$$

with $N=M_{t}(x)$. Indeed, notice that (5.8) gives

$$
|\sigma| \leqslant L \inf _{\rho \in D} \Lambda(M \cdot(\cdot), \rho)\left|\mathrm{D}^{s} \boldsymbol{b}\right|
$$

for any countable set $D$ of kernels $\rho$, and the continuity of $\rho \mapsto \Lambda(N, \rho)$ w.r.t. the $W^{1,1}\left(B_{1}\right)$-norm and the separability of $W^{1,1}\left(B_{1}\right)$ give

$$
|\sigma| \leqslant L G(M .(\cdot))\left|D^{s} \boldsymbol{b}\right| \text {. }
$$

Notice now that the assumption that $\mathrm{D} \cdot \boldsymbol{b}_{t} \ll \mathcal{L}^{d}$ for $\mathcal{L}^{1}$-a.e. $t \in(0, T)$ gives

$$
\operatorname{tr} M_{t}(x)\left|\mathrm{D}^{s} \boldsymbol{b}_{t}\right|=0 \quad \text { for } \mathcal{L}^{1} \text {-a.e. } t \in(0, T) .
$$

Hence, recalling the definition of $\left|\mathrm{D}^{s} \boldsymbol{b}\right|$, the trace of $M_{t}(x)$ vanishes for $\left|\mathrm{D}^{s} \boldsymbol{b}\right|$-a.e. $(t, x)$. Applying the following lemma, courtesy of Alberti, and using (5.10), we obtain that $\sigma=0$, thus concluding the proof.

LEMma 5.2 (Alberti). For any $d \times d$ matrix $N$, the infimum in (5.9) is $|\operatorname{tr} N|$.

Proof. Since $\int\langle N z, \nabla \rho(z)\rangle \mathrm{d} z=-\operatorname{tr} N$, we have to build kernels $\rho$ in such a way that the field $N z$ is as tangential as possible to the level sets of $\rho$. Notice first that the lower bound follows immediately by the identity

$$
\int_{\mathbb{R}^{d}}\langle N z, \nabla \rho(z)\rangle \mathrm{d} z=\int_{\mathbb{R}^{d}}-\rho(z) \operatorname{div} N z+\operatorname{div}(\rho(z) N z) \mathrm{d} z=-\operatorname{tr} N .
$$

Hence, we only have to show the upper bound. Again, by the identity

$$
\langle N z, \nabla \rho(z)\rangle=\operatorname{div}(N z \rho(z))-\operatorname{tr} N \rho(z),
$$

it suffices to show that, for any $T>0$, there exists $\rho$ such that

$$
\int_{\mathbb{R}^{d}}|\operatorname{div}(N z \rho(z))| \mathrm{d} z \leqslant \frac{2}{T} .
$$


The heuristic idea is (again) to build $\rho$ as the superposition of elementary probability measures associated with the curves $\mathrm{e}^{t N} x, 0 \leqslant t \leqslant T$, on which the divergence operator can be easily estimated. Given a smooth convolution kernel $\theta$ with compact support, it turns out that the function

$$
\rho(z):=\frac{1}{T} \int_{0}^{T} \theta\left(\mathrm{e}^{-t N} z\right) \mathrm{e}^{-t \operatorname{tr} N} \mathrm{~d} t
$$

has the required properties (here $\mathrm{e}^{t N} x=\sum_{i} t^{i} N^{i} x / i$ ! is the solution of the ODE $\dot{\gamma}=N \gamma$ with the initial condition $\gamma(0)=x)$. Indeed, it is immediate to check that $\rho$ is smooth and compactly supported. To estimate the divergence of $N z \rho(z)$, we note that $\rho=\int \theta(x) \mu_{x} \mathrm{~d} x$, where $\mu_{x}$ are the probability one-dimensional measures concentrated on the image of the curves $t \mapsto \mathrm{e}^{t N} x$ defined by

$$
\mu_{x}:=\left(\mathrm{e}^{\cdot N} x\right)_{\#}\left(\frac{1}{T} \mathcal{L}^{1}\llcorner[0, T]) .\right.
$$

Indeed, for any $\varphi \in C_{\mathrm{c}}^{\infty}\left(\mathbb{R}^{d}\right)$, we have

$$
\begin{aligned}
\int_{\mathbb{R}^{d}} \theta(x)\left\langle\mu_{x}, \varphi\right\rangle \mathrm{d} x & =\frac{1}{T} \int_{0}^{T} \int_{\mathbb{R}^{d}} \theta(x) \varphi\left(\mathrm{e}^{t N} x\right) \mathrm{d} x \mathrm{~d} t \\
& =\frac{1}{T} \int_{0}^{T} \int_{\mathbb{R}^{d}} \theta\left(\mathrm{e}^{-t N} y\right) \mathrm{e}^{-t \operatorname{tr} N} \varphi(y) \mathrm{d} y \mathrm{~d} t \\
& =\int_{\mathbb{R}^{d}} \rho(y) \varphi(y) \mathrm{d} y .
\end{aligned}
$$

By the linearity of the divergence operator, it suffices to check that

$$
\left|\mathrm{D}_{z} \cdot\left(N z \mu_{x}\right)\right|\left(\mathbb{R}^{d}\right) \leqslant \frac{2}{T} \quad \forall x \in \mathbb{R}^{d} .
$$

But this is elementary, since

$$
\int_{\mathbb{R}^{d}}\langle N z, \nabla \varphi(z)\rangle \mathrm{d} \mu_{x}(z)=\frac{1}{T} \int_{0}^{T}\left\langle N \mathrm{e}^{t N} x, \nabla \varphi\left(\mathrm{e}^{t N} x\right)\right\rangle \mathrm{d} t=\frac{\varphi\left(\mathrm{e}^{T N} x\right)-\varphi(x)}{T}
$$

for any $\varphi \in C_{\mathrm{c}}^{\infty}\left(\mathbb{R}^{d}\right)$, so $T \mathrm{D}_{z} \cdot\left(N z \mu_{x}\right)=\delta_{x}-\delta_{\mathrm{e}^{T N} x}$.

The original argument in [9] was slightly different and used, instead of lemma 5.2, a much deeper result, still due to Alberti, saying that, for a $\mathrm{BV}_{\text {loc }}$ function $u: \mathbb{R}^{d} \rightarrow$ $\mathbb{R}^{m}$, the matrix $M(x)$ in the polar decomposition $\mathrm{D} u=M|\mathrm{D} u|$ has rank 1 for $\left|\mathrm{D}^{s} u\right|$-a.e. $x$, i.e. there exist unit vectors $\xi(x) \in \mathbb{R}^{d}$ and $\eta(x) \in \mathbb{R}^{m}$ such that $M(x) z=\eta(x)\langle z, \xi(x)\rangle$. In this case the asymptotically optimal kernels are much easier to build, just by mollifying in the $\xi$ direction much faster than in all other ones. This is precisely what Bouchut and Lions did in some particular cases (respectively, 'Hamiltonian' vector fields and piecewise Sobolev ones).

As in the Sobolev case, from the general theory given in $\S 3$, we can now obtain existence and uniqueness of $\mathcal{L}$-Lagrangian flows, with $\mathcal{L}=L^{\infty}\left(L^{1}\right) \cap L^{\infty}\left(L^{\infty}\right)$ : we just replace in the statement of theorem 4.8 the assumption

$$
\boldsymbol{b} \in L^{1}\left([0, T] ; W_{\mathrm{loc}}^{1,1}\left(\mathbb{R}^{d} ; \mathbb{R}^{d}\right)\right) \quad \text { with } \quad \boldsymbol{b} \in L^{1}\left([0, T] ; \mathrm{BV}_{\mathrm{loc}}\left(\mathbb{R}^{d} ; \mathbb{R}^{d}\right)\right)
$$

assuming as usual that $\mathrm{D} \cdot \boldsymbol{b}_{t} \ll \mathcal{L}^{d}$ for $\mathcal{L}^{1}$-a.e. $t \in[0, T]$. 
Analogously, with the same replacements in theorem 4.10 (for $\boldsymbol{b}$ and $\boldsymbol{b}^{h}$ ), we obtain stability of $\mathcal{L}$-Lagrangian flows.

\section{Quantitative ODE estimates: $W^{1, p}$ regularity with $p>1$}

In this section and the next we introduce a further approach to the well-posedness of the ODE, based on quantitative a priori estimates, as an alternative to the approach exploiting the well-posedness of the continuity equation (in the various regularity contexts) and the link between the ODE and the continuity equation described in $\S 3$.

Here we restrict our attention to $\mathcal{L}$-Lagrangian flows with $\mathcal{L}:=L^{\infty}\left(L^{\infty}\right)$ and for simplicity we refer to them (in this and in the next section) simply as regular Lagrangian flows. We recall that, given a vector field $\boldsymbol{b}:[0, T] \times \mathbb{R}^{d} \rightarrow \mathbb{R}^{d}$, we are considering as 'admissible solutions to the ODE' those maps $\boldsymbol{X}:[0, T] \times \mathbb{R}^{d} \rightarrow \mathbb{R}^{d}$ such that:

(i) for $\mathcal{L}^{d}$-a.e. $x \in \mathbb{R}^{d}$, the function $t \mapsto \boldsymbol{X}(t, x)$ is a solution of the ODE in the integral sense;

(ii) there exists a constant $L>0$ such that $X(t, \cdot)_{\#} \mathcal{L}^{d} \leqslant L \mathcal{L}^{d}$ for all $t \in[0, T]$.

The constant $L$ in (ii) is called the compressibility constant of the flow $\boldsymbol{X}$.

For simplicity, in this and in the next section we only consider globally bounded vector fields: the extension to more general growth conditions does not pose a problem, but makes some of the computations much longer. At many occurrences we will also avoid explicitly indicating the time dependence. We deal with three different regularity classes: $W^{1, p}$ with $p>1$ (in this section), $W^{1,1}$ and the case in which the derivative is a singular integral of an $L^{1}$ function (in the next section). We remark that we do not systematically assume conditions about the spatial divergence $\operatorname{div} \boldsymbol{b}$ : divergence bounds will be needed for the existence result only.

The objective is to develop in the non-Lipschitz context a theory for the ODE that is 'parallel' to the usual Cauchy-Lipschitz theory for the Lipschitz context, and which is independent from the PDE theory. In particular, this approach allows us to recover the following for the ODE:

- existence and uniqueness;

- stability (with respect to approximations of the vector field), with an explicit convergence rate;

- compactness, under natural bounds (this is relevant in view of applications to nonlinear PDEs);

- regularity of the flow $\boldsymbol{X}(t, x)$ with respect to the initial position $x$ (this also gives some mild propagation of regularity for the transport equation).

The presentation is based on $[37,61]$. Some ideas originated from $[19,83]$. 


\subsection{An integral quantity and the lower bound}

Remember the very basic estimate providing (2.1) in the context of Lipschitz vector fields. A variant of it can be performed using the following formal estimate for the logarithm of the spatial derivative of the flow:

$$
\frac{\mathrm{d}}{\mathrm{d} t} \log |\nabla \boldsymbol{X}| \leqslant \frac{1}{|\nabla \boldsymbol{X}|}\left|\frac{\mathrm{d}}{\mathrm{d} t} \nabla \boldsymbol{X}\right|=\frac{1}{|\nabla \boldsymbol{X}|}|\nabla(\boldsymbol{b}(\boldsymbol{X}))|=|\nabla \boldsymbol{b}|(\boldsymbol{X}) .
$$

Again, when $\boldsymbol{b}$ is Lipschitz, we recover that the flow is Lipschitz with a Lipschitz constant depending exponentially on the Lipschitz constant of $\boldsymbol{b}$.

The relevance of (6.1) is that it admits an integral version, which works well even out of the Lipschitz case in some weak regularity contexts.

Let us fix two regular Lagrangian flows $\boldsymbol{X}_{1}$ and $\boldsymbol{X}_{2}$, associated with the vector fields $\boldsymbol{b}_{1}$ and $\boldsymbol{b}_{2}$, respectively, and with compressibility constants $L_{1}$ and $L_{2}$. Given a (small) parameter $\delta>0$ and a truncation radius $R>0$, we consider the following (time-dependent) quantity:

$$
\Phi_{\delta}(t)=\int_{B_{R}} \log \left(1+\frac{\left|\boldsymbol{X}_{1}(t, x)-\boldsymbol{X}_{2}(t, x)\right|}{\delta}\right) \mathrm{d} x .
$$

We start by establishing a simple, but useful, lower bound for $\Phi_{\delta}$. Given an arbitrary $\gamma>0$ we can compute

$\Phi_{\delta}(t) \geqslant \int_{B_{R} \cap\left\{\left|\boldsymbol{X}_{1}-\boldsymbol{X}_{2}\right|>\gamma\right\}} \log \left(1+\frac{\gamma}{\delta}\right) \mathrm{d} x=\mathcal{L}^{d}\left(B_{R} \cap\left\{\left|\boldsymbol{X}_{1}-\boldsymbol{X}_{2}\right|>\gamma\right\}\right) \log \left(1+\frac{\gamma}{\delta}\right)$.

In particular, in the special case $\boldsymbol{b}_{1}=\boldsymbol{b}_{2}=\boldsymbol{b}$ (in which $\Phi_{\delta}$ is somehow 'measuring the non-uniqueness'), we deduce that, in order to have non-uniqueness, $\Phi_{\delta}$ must blow up at least as fast as $\log (1 / \delta)$ as $\delta \rightarrow 0$. More generally, we understand that upper bounds on $\Phi_{\delta}$ will provide upper estimates on the measure of the superlevels $\left\{\left|\boldsymbol{X}_{1}-\boldsymbol{X}_{2}\right|>\gamma\right\}$.

\subsection{The maximal function}

Since we have now dropped the Lipschitz context, the difference quotients

$$
\frac{|\boldsymbol{b}(x)-\boldsymbol{b}(y)|}{|x-y|}
$$

are no longer uniformly bounded. In the proof of the upper bounds for the functional $\Phi_{\delta}$ we will again have to deal with difference quotients, but we will take advantage of the fact that they will always appear under the integral sign.

Suitable estimates can be provided using the classical maximal function. Given $f \in L_{\text {loc }}^{1}\left(\mathbb{R}^{d} ; \mathbb{R}^{m}\right)$, we define its maximal function as

$$
M f(x)=\sup _{r>0} f_{B(x, r)}|f(y)| \mathrm{d} y, \quad x \in \mathbb{R}^{d} .
$$

Similarly, when $\mu$ is an $\mathbb{R}^{m}$-valued measure in $\mathbb{R}^{d}$ with locally finite total variation, we define

$$
M \mu(x)=\sup _{r>0} \frac{|\mu|(B(x, r))}{\mathcal{L}^{d}(B(x, r))}, \quad x \in \mathbb{R}^{d} .
$$


Since $\left|f \mathcal{L}^{d}\right|=|f| \mathcal{L}^{d}$, the two definitions are consistent; namely, $M\left(f \mathcal{L}^{d}\right)=M f$. Note that, if $f \in L^{1}\left(\mathbb{R}^{d} ; \mathbb{R}^{m}\right)$, or if $\mu$ is a measure with globally finite total variation in $\mathbb{R}^{d}$, then the maximal function is $\mathcal{L}^{d}$-a.e. finite.

A fundamental question is to provide bounds for this operator. It is immediate to see that

$$
\|M f\|_{L^{\infty}} \leqslant\|f\|_{L^{\infty}} .
$$

Remarkably, the analogue property involving $L^{1}$ does not hold. Only the weak estimate

$$
\|M f\|_{M^{1}} \leqslant C_{d, 1}\|f\|_{L^{1}}
$$

holds, where the weak Lebesgue space $M^{1}\left(\mathbb{R}^{d}\right)$ is defined as the space consisting of all measurable functions $g$ on $\mathbb{R}^{d}$ such that

$$
\|g\|_{M^{1}}=\sup _{\lambda>0}\left\{\lambda \mathcal{L}^{d}(\{x:|g(x)|<\lambda\})\right\}<\infty .
$$

Note carefully that the quantity $\|g\|_{M^{1}}$ is not a norm, and for this reason we have chosen the notation with the triple vertical bar.

By interpolating (6.6) and (6.7) we can obtain the strong estimate

$$
\|M f\|_{L^{p}} \leqslant C_{d, p}\|f\|_{L^{p}},
$$

valid for every $1<p \leqslant \infty$, with a constant $C_{d, p}$ that blows up as $p \rightarrow 1$.

The maximal function is relevant in our context because of the following property. Assume that $f \in B V\left(\mathbb{R}^{d}\right)$. There then exists a Lebesgue negligible set $N \subset \mathbb{R}^{d}$ such that

$$
|f(x)-f(y)| \leqslant C|x-y|(M D f(x)+M D f(y)) \quad \forall x, y \in \mathbb{R}^{d} \backslash N,
$$

where $C$ is a dimensional constant. Estimate (6.9) provides a control of the difference quotients with the maximal function of the derivative. We shall exploit this property instead of the Lipschitz assumption in the estimates for $\Phi_{\delta}$.

ExERCise 6.1. Prove formula (6.9) (a detailed proof is given, for instance, in [16, theorem 5.34]).

For a detailed exposition of the theory of maximal functions we refer the reader to, for instance, [98].

\subsection{Upper bound for the integral quantity}

We derive now an upper bound on $\Phi_{\delta}$ that makes use of the dynamics of our situation; that is, we start to exploit the fact that $\boldsymbol{X}_{1}$ and $\boldsymbol{X}_{2}$ are regular Lagrangian flows. The following estimates will rely on the $W^{1, p}$ regularity of the vector fields $\boldsymbol{b}_{1}$ and $\boldsymbol{b}_{2}$ (we assume $p>1$ in this section, while $p=1$ will be considered in the next section), and on the compressibility constants of the regular Lagrangian flows. Also notice that, if $x \in B_{R}$, then $\boldsymbol{X}_{i}(t, x) \in B_{R+T\left\|\boldsymbol{b}_{i}\right\|_{\infty}}$ for all $t \in[0, T]$. 
We estimate the time derivative of $\Phi_{\delta}$ as follows:

$$
\begin{aligned}
& \Phi_{\delta}^{\prime}(t) \leqslant \int_{B_{R}} \frac{\left|\boldsymbol{b}_{1}\left(\boldsymbol{X}_{1}\right)-\boldsymbol{b}_{2}\left(\boldsymbol{X}_{2}\right)\right|}{\delta+\left|\boldsymbol{X}_{1}-\boldsymbol{X}_{2}\right|} \mathrm{d} x \\
& \leqslant \int_{B_{R}} \frac{\left|\boldsymbol{b}_{1}\left(\boldsymbol{X}_{2}\right)-\boldsymbol{b}_{2}\left(\boldsymbol{X}_{2}\right)\right|}{\delta+\left|\boldsymbol{X}_{1}-\boldsymbol{X}_{2}\right|} \mathrm{d} x+\int_{B_{R}} \frac{\left|\boldsymbol{b}_{1}\left(\boldsymbol{X}_{1}\right)-\boldsymbol{b}_{1}\left(\boldsymbol{X}_{2}\right)\right|}{\delta+\left|\boldsymbol{X}_{1}-\boldsymbol{X}_{2}\right|} \mathrm{d} x \\
& \leqslant \frac{1}{\delta} \int_{B_{R}}\left|\boldsymbol{b}_{1}\left(\boldsymbol{X}_{2}\right)-\boldsymbol{b}_{2}\left(\boldsymbol{X}_{2}\right)\right| \mathrm{d} x+\int_{B_{R}} \min \left\{\frac{2\left\|\boldsymbol{b}_{1}\right\|_{\infty}}{\delta} ; \frac{\left|\boldsymbol{b}_{1}\left(\boldsymbol{X}_{1}\right)-\boldsymbol{b}_{1}\left(\boldsymbol{X}_{2}\right)\right|}{\left|\boldsymbol{X}_{1}-\boldsymbol{X}_{2}\right|}\right\} \mathrm{d} x \\
& \leqslant \frac{L_{2}}{\delta}\left\|\boldsymbol{b}_{1}-\boldsymbol{b}_{2}\right\|_{L^{1}\left(B_{R+T\left\|\boldsymbol{b}_{2}\right\|_{\infty}}\right)} \\
& +\int_{B_{R}} \min \left\{\frac{2\left\|\boldsymbol{b}_{1}\right\|_{\infty}}{\delta} ; C\left(M \nabla \boldsymbol{b}_{1}\left(\boldsymbol{X}_{1}\right)+M \nabla \boldsymbol{b}_{1}\left(\boldsymbol{X}_{2}\right)\right)\right\} \mathrm{d} x .
\end{aligned}
$$

We can estimate

$$
\int_{B_{R}} M \nabla \boldsymbol{b}_{1}\left(\boldsymbol{X}_{i}\right) \mathrm{d} x \leqslant L_{i} \int_{B_{R+T\left\|\boldsymbol{b}_{i}\right\| \infty}} M \nabla \boldsymbol{b}_{1}(x) \mathrm{d} x \leqslant C\left\|M \nabla \boldsymbol{b}_{1}\right\|_{L^{p}} \leqslant C\left\|\nabla \boldsymbol{b}_{1}\right\|_{L^{p}},
$$

where the constant $C$ depends on $L_{i}, p,\left\|\boldsymbol{b}_{i}\right\|_{\infty}, R$ and $T$. Note that with a bit more care this estimate could be made local, so as to depend on the norm of $\nabla \boldsymbol{b}_{1}$ on a compact set only.

For a vector field $\boldsymbol{b}_{1} \in W^{1, p}$ with $p>1$ we hence deduce

$$
\Phi_{\delta}^{\prime}(t) \leqslant \frac{C}{\delta}\left\|\boldsymbol{b}_{1}-\boldsymbol{b}_{2}\right\|_{L_{x}^{1}}+C\left\|\nabla \boldsymbol{b}_{1}\right\|_{L_{x}^{p}}
$$

and so, since $\Phi_{\delta}(0)=0$, we have

$$
\Phi_{\delta}(t) \leqslant \frac{C}{\delta}\left\|\boldsymbol{b}_{1}-\boldsymbol{b}_{2}\right\|_{L_{t}^{1}\left(L_{x}^{1}\right)}+C\left\|\nabla \boldsymbol{b}_{1}\right\|_{L_{t}^{1}\left(L_{x}^{p}\right)} .
$$

Putting this together with (6.3), we conclude

$$
\begin{aligned}
& \mathcal{L}^{d}\left(B_{R} \cap\left\{\left|\boldsymbol{X}_{1}(t, \cdot)-\boldsymbol{X}_{2}(t, \cdot)\right|>\gamma\right\}\right) \\
& \quad \leqslant \frac{C}{\delta \log (1+\gamma / \delta)}\left\|\boldsymbol{b}_{1}-\boldsymbol{b}_{2}\right\|_{L_{t}^{1}\left(L_{x}^{1}\right)}+\frac{C}{\log (1+\gamma / \delta)}\left\|\nabla \boldsymbol{b}_{1}\right\|_{L_{t}^{1}\left(L_{x}^{p}\right)} .
\end{aligned}
$$

This is the fundamental estimate, which will allow us to deduce many of the wellposedness results for the ODE.

\subsection{Well-posedness of the ODE: $W^{1, p}$ regularity}

We start with uniqueness, which is an immediate consequence of (6.11). Since we consider the case in which $\boldsymbol{b}_{1}=\boldsymbol{b}_{2}$, we have

$$
\mathcal{L}^{d}\left(B_{R} \cap\left\{\left|\boldsymbol{X}_{1}(t, \cdot)-\boldsymbol{X}_{2}(t, \cdot)\right|>\gamma\right\}\right) \leqslant \frac{C}{\log (1+\gamma / \delta)}\left\|\nabla \boldsymbol{b}_{1}\right\|_{L_{t}^{1}\left(L_{x}^{p}\right)}
$$

for every $\delta>0$ and every $R>0$. It suffices to let $\delta \rightarrow 0$.

Next we discuss stability. Consider $\boldsymbol{b} \in L_{t}^{1}\left(W_{x}^{1, p}\right)$ and a sequence $\left(\boldsymbol{b}_{n}\right)$ convergent to $\boldsymbol{b}$ in $L_{\text {loc }}^{1}$, equibounded in $L^{\infty}$. Assume that the regular Lagrangian flows $\boldsymbol{X}$ 
and $\boldsymbol{X}_{n}$ have equibounded compressibility constants. The fundamental estimate (6.11) now reads

$$
\begin{aligned}
& \mathcal{L}^{d}\left(B_{R} \cap\left\{\left|\boldsymbol{X}_{n}(t, \cdot)-\boldsymbol{X}(t, \cdot)\right|>\gamma\right\}\right) \\
& \quad \leqslant \frac{C}{\delta \log (1+\gamma / \delta)}\left\|\boldsymbol{b}_{n}-\boldsymbol{b}\right\|_{L_{t}^{1}\left(L_{x, \text { loc }}^{1}\right)}+\frac{C}{\log (1+\gamma / \delta)}\|\nabla \boldsymbol{b}\|_{L_{t}^{1}\left(L_{x}^{p}\right)} \\
& \quad=\mathrm{I}+\mathrm{II} .
\end{aligned}
$$

Given $\gamma, \eta>0$, we choose $\delta>0$ so small that $\mathrm{II} \leqslant \eta / 2$. This fixes the quantity $C / \delta \log (1+\gamma / \delta)$ in I. We can therefore find $\bar{n}$ so large that $\mathrm{I} \leqslant \eta / 2$ for all $n \geqslant \bar{n}$.

We have hence discovered that: given $\gamma>0$ and $R>0$, for every $\eta>0$ we can find $\bar{n}$ such that

$$
\mathcal{L}^{d}\left(B_{R} \cap\left\{\left|\boldsymbol{X}_{n}(t, \cdot)-\boldsymbol{X}(t, \cdot)\right|>\gamma\right\}\right) \leqslant \eta \quad \forall n \geqslant \bar{n} .
$$

This means that $\boldsymbol{X}_{n}$ is converging to $\boldsymbol{X}$ locally in measure in $\mathbb{R}^{d}$. Since $\boldsymbol{X}$ and $\boldsymbol{X}_{n}$ are locally equibounded, this also implies $L_{\text {loc }}^{1}$ convergence: given $R>0$, for every $\eta>0$ we can find $\bar{n}$ such that

$$
\int_{B_{R}}\left|\boldsymbol{X}_{n}(t, x)-\boldsymbol{X}(t, x)\right| \mathrm{d} x \leqslant \eta \quad \forall n \geqslant \bar{n}
$$

Note the important fact that stability comes with a quantitative rate: the value $\bar{n}$ depends only on $\eta$ and on the equibounds on the sequence.

The proof of the compactness goes along the same line. Consider a sequence $\left(\boldsymbol{b}_{n}\right)$ that is equibounded in $L^{\infty}$ and in $L_{t}^{1}\left(W_{x}^{1, p}\right)$. Assume that there exist associated regular Lagrangian flows $\boldsymbol{X}_{n}$ with equibounded compressibility constants. We can then use the fundamental estimate (6.11) to write

$$
\begin{aligned}
& \mathcal{L}^{d}\left(B_{R} \cap\left\{\left|\boldsymbol{X}_{n}(t, \cdot)-\boldsymbol{X}_{m}(t, \cdot)\right|>\gamma\right\}\right) \\
& \quad \leqslant \frac{C}{\delta \log (1+\gamma / \delta)}\left\|\boldsymbol{b}_{n}-\boldsymbol{b}_{m}\right\|_{L_{t}^{1}\left(L_{x}^{1}\right)}+\frac{C}{\log (1+\gamma / \delta)} \\
& \quad=\mathrm{I}+\mathrm{II},
\end{aligned}
$$

where the constant $C$ in II also depends on the equibounds on $\left\|D \boldsymbol{b}_{n}\right\|_{L_{t}^{1}\left(L_{x}^{p}\right)}$.

Given $\eta>0$, we find $\delta>0$ such that II $\leqslant \eta / 2$. Correspondingly, we find $\bar{n}$ such that $\mathrm{I} \leqslant \eta / 2$ for every $n, m \geqslant \bar{n}$.

Summarizing, we have proven that, given $\gamma>0$ and $R>0$, for every $\eta>0$ we can find $\bar{n}$ such that

$$
\int_{B_{R}}\left|\boldsymbol{X}_{n}(t, x)-\boldsymbol{X}_{m}(t, x)\right| \mathrm{d} x \leqslant \eta \quad \forall n, m \geqslant \bar{n},
$$

that is, the sequence $\left(\boldsymbol{X}_{n}\right)$ is locally precompact in measure. As for the stability, thanks to the local equiboundedness of $\boldsymbol{X}_{n}$, precompactness in $L_{\text {loc }}^{1}$ can be deduced from this.

REMARK 6.2. The analogue compactness statement under $L^{1}$ bounds on $\nabla \boldsymbol{b}_{n}$, instead of $L^{p}$ bounds, is the content of a conjecture due to Bressan [45]. The $W^{1,1}$ theory presented in the next section will replace the $L^{p}$ bounds on $\nabla \boldsymbol{b}_{n}$ with equiintegrability bounds. 
The compactness is the key property required in order to prove existence of the regular Lagrangian flow associated with a bounded vector field $\boldsymbol{b} \in L_{t}^{1}\left(W_{x}^{1, p}\right)$. At this point, we have to assume some condition about the divergence of $\boldsymbol{b}$ and, for simplicity, we assume $\operatorname{div} \boldsymbol{b} \in L^{\infty}$.

We regularize $\boldsymbol{b}$ by convolution in order to get a sequence $\boldsymbol{b}_{n}$ of smooth vector fields that are equibounded in $L^{\infty} \cap L_{t}^{1}\left(W_{x}^{1, p}\right)$, and with $\operatorname{div} \boldsymbol{b}_{n}$ equibounded in $L^{\infty}$. Remembering (2.6), we infer that the (classical) flows associated with $\boldsymbol{b}_{n}$ have equibounded compressibility constants. We are therefore in position to apply the compactness property, and it is simple to prove that this produces a regular Lagrangian flow associated with $\boldsymbol{b}$.

\subsection{A mild regularity property of the regular Lagrangian flow}

Remember that, for Lipschitz vector fields, the flow inherits regularity with respect to the spatial variable: $x \mapsto \boldsymbol{X}(t, x)$ is Lipschitz, with the Lipschitz constant depending exponentially on the time and on the Lipschitz constant of the vector field (cf. (2.1)).

We now want to derive a similar result for Sobolev vector fields. In order to do this, we consider a functional $\Psi_{\delta}$ strictly related to the functional $\Phi_{\delta}(t)$ in $(6.2)$, that is

$$
\Psi(\boldsymbol{X})=\left\|\sup _{0 \leqslant t \leqslant T} \sup _{r>0} f_{B(x, r)} \log \left(1+\frac{|\boldsymbol{X}(t, x)-\boldsymbol{X}(t, y)|}{r}\right) \mathrm{d} y\right\|_{L_{x}^{p}\left(B_{R}\right)},
$$

where $R>0$ is fixed.

We want to derive upper bounds for the functional $\Psi(\boldsymbol{X})$. We begin by differentiating with respect to time the integral:

$$
\begin{aligned}
\frac{\mathrm{d}}{\mathrm{d} t} f_{B(x, r)} \log & \left(1+\frac{|\boldsymbol{X}(t, x)-\boldsymbol{X}(t, y)|}{r}\right) \mathrm{d} y \\
& \leqslant f_{B(x, r)} \frac{|\boldsymbol{b}(\boldsymbol{X}(t, x))-\boldsymbol{b}(\boldsymbol{X}(t, y))|}{|\boldsymbol{X}(t, x)-\boldsymbol{X}(t, y)|} \mathrm{d} y \\
& \leqslant C f_{B(x, r)}[M \nabla \boldsymbol{b}(\boldsymbol{X}(t, x))+M \nabla \boldsymbol{b}(\boldsymbol{X}(t, y))] \mathrm{d} y \\
& =C M \nabla \boldsymbol{b}(\boldsymbol{X}(t, x))+C f_{B(x, r)} M \nabla \boldsymbol{b}(\boldsymbol{X}(t, y)) \mathrm{d} y .
\end{aligned}
$$

Integrating in time we deduce

$$
\begin{aligned}
f_{B(x, r)} \log & \left(1+\frac{|\boldsymbol{X}(t, x)-\boldsymbol{X}(t, y)|}{r}\right) \mathrm{d} y \\
\leqslant & f_{B(x, r)} \log \left(1+\frac{|x-y|}{r}\right) \mathrm{d} y+C \int_{0}^{t} M \nabla \boldsymbol{b}(\boldsymbol{X}(s, x)) \mathrm{d} s \\
& +C \int_{0}^{t} f_{B(x, r)} M \nabla \boldsymbol{b}(\boldsymbol{X}(s, y)) \mathrm{d} y \mathrm{~d} s \\
\leqslant & \log 2+C \int_{0}^{t} M \nabla \boldsymbol{b}(\boldsymbol{X}(s, x)) \mathrm{d} s+C \int_{0}^{t} f_{B(x, r)} M \nabla \boldsymbol{b}(\boldsymbol{X}(s, y)) \mathrm{d} y \mathrm{~d} s .
\end{aligned}
$$


Taking the supremum over $0 \leqslant t \leqslant T$ and over $r>0$, and finally the $L_{x}^{p}\left(B_{R}\right)$-norm, we deduce

$$
\begin{aligned}
\Psi(\boldsymbol{X}) \leqslant & +C \int_{0}^{T}\|M \nabla \boldsymbol{b}(\boldsymbol{X}(s, x))\|_{L^{p}\left(B_{R}\right)} \mathrm{d} s \\
& +C \int_{0}^{T}\|M[M \nabla \boldsymbol{b}(\boldsymbol{X}(s, \cdot))]\|_{L^{p}\left(B_{R}\right)} \mathrm{d} s \\
\leqslant & +C \int_{0}^{T}\|M \nabla \boldsymbol{b}\|_{L^{p}} \mathrm{~d} s+C \int_{0}^{T}\|M \nabla \boldsymbol{b}(\boldsymbol{X}(s, \cdot))\|_{L^{p}} \mathrm{~d} s \\
\leqslant & +C\|\nabla \boldsymbol{b}\|_{L_{t}^{1}\left(L_{x}^{p}\right)}+C \int_{0}^{T}\|M \nabla \boldsymbol{b}\|_{L^{p}} \mathrm{~d} s \\
\leqslant & +C\|\nabla \boldsymbol{b}\|_{L_{t}^{1}\left(L_{x}^{p}\right)} \\
\leqslant & C,
\end{aligned}
$$

where the constant $C$ only depends on the bounds on $\boldsymbol{b}$ and on the compressibility constant of the regular Lagrangian flow $\boldsymbol{X}$.

We shall use Chebyshev's inequality which asserts that, for every $1 \leqslant p<\infty$ and every $\lambda>0$, we can estimate

$$
\mathcal{L}^{d}(\{|f|>\lambda\}) \leqslant \frac{\|f\|_{L^{p}}^{p}}{\lambda^{p}} .
$$

Applying this with $\lambda=C / \varepsilon^{1 / p}$ to the function of $x$ introduced in (6.12), we obtain the existence of a set $K \subset B_{R}$ with $\mathcal{L}^{d}\left(B_{R} \backslash K\right) \leqslant \varepsilon$ such that

$$
\sup _{0 \leqslant t \leqslant T} \sup _{r>0} f_{B(x, r)} \log \left(1+\frac{|\boldsymbol{X}(t, x)-\boldsymbol{X}(t, y)|}{r}\right) \mathrm{d} y \leqslant \frac{C}{\varepsilon^{1 / p}} \quad \forall x \in K .
$$

This means that

$$
f_{B(x, r)} \log \left(1+\frac{|\boldsymbol{X}(t, x)-\boldsymbol{X}(t, y)|}{r}\right) \mathrm{d} y \leqslant \frac{C}{\varepsilon^{1 / p}}
$$

for every $x \in K$, every $r>0$ and every $0 \leqslant t \leqslant T$.

We apply this in order to derive the Lipschitz estimate for the regular Lagrangian flow $\boldsymbol{X}$. Let $x$ and $x^{\prime}$ be points of $K$ and set $r=\left|x-x^{\prime}\right|$. Define

$$
C_{x, x^{\prime}}=B(x, r) \cap B\left(x^{\prime}, r\right) .
$$

Notice that $\mathcal{L}^{d}\left(C_{x, x^{\prime}}\right)=c_{d} r^{d}$ for a positive dimensional constant $c_{d}$. We can estimate

$$
\begin{aligned}
\log \left(1+\frac{\left|\boldsymbol{X}(t, x)-\boldsymbol{X}\left(t, x^{\prime}\right)\right|}{r}\right)= & f_{C_{x, x^{\prime}}} \log \left(1+\frac{\left|\boldsymbol{X}(t, x)-\boldsymbol{X}\left(t, x^{\prime}\right)\right|}{r}\right) \mathrm{d} y \\
\leqslant & f_{C_{x, x^{\prime}}} \log \left(1+\frac{|\boldsymbol{X}(t, x)-\boldsymbol{X}(t, y)|}{r}\right) \mathrm{d} y \\
& +f_{C_{x, x^{\prime}}} \log \left(1+\frac{\left|\boldsymbol{X}\left(t, x^{\prime}\right)-\boldsymbol{X}(t, y)\right|}{r}\right) \mathrm{d} y
\end{aligned}
$$




$$
\begin{aligned}
\leqslant & C f_{B(x, r)} \log \left(1+\frac{|\boldsymbol{X}(t, x)-\boldsymbol{X}(t, y)|}{r}\right) \mathrm{d} y \\
& +C f_{B\left(x^{\prime}, r\right)} \log \left(1+\frac{\left|\boldsymbol{X}\left(t, x^{\prime}\right)-\boldsymbol{X}(t, y)\right|}{r}\right) \mathrm{d} y \\
\leqslant & \frac{C}{\varepsilon^{1 / p}} .
\end{aligned}
$$

As a consequence

$$
\left|\boldsymbol{X}(t, x)-\boldsymbol{X}\left(t, x^{\prime}\right)\right| \leqslant\left|x-x^{\prime}\right| \exp \left(\frac{C}{\varepsilon^{1 / p}}\right)
$$

for every $x$ and $x^{\prime}$ belonging to $K$, that is

$$
\left.\operatorname{Lip} \boldsymbol{X}(t, \cdot)\right|_{K} \leqslant \exp \left(\frac{C}{\varepsilon^{1 / p}}\right) .
$$

Remember that the set $K \subset B_{R}$ is such that $\mathcal{L}^{d}\left(B_{R} \backslash K\right) \leqslant \varepsilon$.

REMARK 6.3. The Lipschitz property we have proven is usually called a Lusin-type approximation with Lipschitz function. From this, approximate differentiability $\mathcal{L}^{d_{-}}$ a.e. of the map $x \mapsto \boldsymbol{X}(t, x)$ can be deduced.

ExERCISE 6.4. Using the Ascoli-Arzelà theorem, find another proof of the compactness of the regular Lagrangian flow. Notice that it is essential that the control on the Lipschitz constant provided by (6.14) is quantitative.

\section{Quantitative ODE estimates: $W^{1,1}$ regularity and singular integrals}

In the previous section we described the quantitative estimates and their consequences in the case of $W^{1, p}$ regularity of the vector field when $p>1$. The case $p=1$ (and a fortiori the BV case) was not covered by this analysis due to the lack of strong estimates for $p=1$ for the maximal function: (6.8) does not hold for $p=1$ and only the weak estimate (6.7) is available. However, (6.7) seems to be of no help for our purposes: we need to bound an integral of difference quotients, which are in turn bounded by the maximal function of the gradient of the vector field. The bound in $M^{1}$ coming from (6.7) does not even guarantee integrability!

The good news is that there is an additional element that can be exploited in our analysis, and this refined proof will allow us to obtain the same theory (except for the regularity results in $\S 6.5$ ) in the $W^{1,1}$ case (but unfortunately not in the BV case, presently only covered by the theory of renormalized solutions).

In the derivation of the upper bound for the functional $\Phi_{\delta}(t)$, after differentiation in time, we obtained the bound

$$
\Phi_{\delta}^{\prime}(t) \leqslant \frac{L_{2}}{\delta}\left\|\boldsymbol{b}_{1}-\boldsymbol{b}_{2}\right\|_{L^{1}}+\int_{B_{R}} \min \left\{\frac{2\left\|b_{1}\right\|_{\infty}}{\delta} ; C\left(M \nabla \boldsymbol{b}_{1}\left(\boldsymbol{X}_{1}\right)+M \nabla \boldsymbol{b}_{1}\left(\boldsymbol{X}_{2}\right)\right)\right\} \mathrm{d} x .
$$

Afterwards, we proceeded with the estimate of the difference quotients, simply 'forgetting' the first term inside the minimum. 
In this section we describe how an interpolation argument (exploiting the $L^{\infty}$ term $2\|\boldsymbol{b}\|_{\infty} / \delta$ in the above minimum) will allow us to recover both the $W^{1,1}$ case and the case in which the derivative of the vector field is a singular integral of an $L^{1}$ function. Note that this second class is not included in, nor includes, BV. We also remark that equi-integrability bounds will play a role in the estimates: this is the obstruction to pushing this $W^{1,1}$ technique to BV.

\subsection{Weak Lebesgue spaces and interpolation}

In order to address the case $p=1$, some additional tools will be needed. Recall that the weak Lebesgue space $M^{1}\left(\mathbb{R}^{d}\right)$ is defined as the space consisting of all measurable functions $g$ on $\mathbb{R}^{d}$ such that

$$
\|g\|_{M^{1}}=\sup _{\lambda>0}\left\{\lambda \mathcal{L}^{d}(\{x:|g(x)|<\lambda\})\right\}<\infty .
$$

We have already noticed that the maximal function satisfies a weak inequality from $L^{1}$ into $M^{1}$, expressed by (6.7). The same holds when we consider the maximal function of a measure, defined as in (6.5):

$$
\|M \mu\|_{M^{1}} \leqslant C_{d, 1}\|\mu\|_{\mathcal{M}},
$$

where on the right-hand side we have the total variation norm.

The space $M^{1}$ is strictly bigger than $L^{1}$. For instance, on the real line, $1 / x$ belongs to $M^{1}$ but fails to be in $L^{1}$ (even locally, due to the blow-up at the origin). The following interpolation inequality may be interpreted by informally saying that ' $M{ }^{1}$ is not too distant from $L^{1}$ :

$$
\|f\|_{L^{1}} \leqslant\|f\|_{M^{1}}\left[1+\log \left(\frac{C\|f\|_{L^{\infty}}}{\|f\|_{M^{1}}}\right)\right] .
$$

Indeed, we can bound the $L^{1}$-norm with the $M^{1}$-(pseudo)norm, up to a logarithm of the $L^{\infty}$-norm. We notice that (7.2) holds for functions defined on a compact set, and that one could similarly interpolate between $M^{1}$ and $L^{p}$ for any $p>1$.

EXERCISE 7.1. Prove the interpolation inequality (7.2). In order to do this, rewrite the $L^{1}$-norm of $f$ as the integral of the distribution function of $f$, defined as usual by $\alpha(\lambda)=\mathcal{L}^{d}(\{|f|>\lambda\})$. Split the integral into the sum of the integrals over $[0, \Lambda]$ and $\left[\Lambda,\|f\|_{L^{\infty}}\right]$, use suitable estimates in the two intervals, and conclude by optimizing over $\Lambda$.

\subsection{Uniqueness for the ODE: $W^{1,1}$ regularity}

We show now how to extend the estimates in $\S 6.3$ to include the case $p=1$. For clearness and simplicity of exposition we focus our presentation on the uniqueness issue, but all the well-posedness results of $\S 6.4$ could be proven in this context along the same lines. However, it is less clear in this case how to extend to the Lipschitz estimates in $\S 6.5$.

Given a vector field $\boldsymbol{b} \in W^{1,1}$, we consider two regular Lagrangian flows $\boldsymbol{X}_{1}$ and $\boldsymbol{X}_{2}$ with compressibility constants given, respectively, by $L_{1}$ and $L_{2}$. Proceeding 
as in (6.10) we estimate

$$
\begin{aligned}
\Phi_{\delta}^{\prime}(t) & \leqslant \int_{B_{R}} \min \left\{\frac{2\|\boldsymbol{b}\|_{\infty}}{\delta} ; C\left(M \mathrm{D} \boldsymbol{b}\left(\boldsymbol{X}_{1}\right)+M \mathrm{D} \boldsymbol{b}\left(\boldsymbol{X}_{2}\right)\right)\right\} \mathrm{d} x \\
& \leqslant C\left(L_{1}+L_{2}\right) \int_{B_{R+T\|\boldsymbol{b}\|_{\infty}}} \min \left\{\frac{\|\boldsymbol{b}\|_{\infty}}{\delta} ; M \mathrm{D} \boldsymbol{b}\right\} \mathrm{d} x .
\end{aligned}
$$

By integrating with respect to time we arrive at

$$
\Phi_{\delta}(t) \leqslant C\left(L_{1}+L_{2}\right) \int_{0}^{T} \int_{B_{R+T\|\boldsymbol{b}\|}} \min \left\{\frac{\|\boldsymbol{b}\|_{\infty}}{\delta} ; M \mathrm{D} \boldsymbol{b}\right\} \mathrm{d} x \mathrm{~d} s .
$$

We now set

$$
\phi(s, x)=\min \left\{\frac{\|\boldsymbol{b}\|_{\infty}}{\delta} ; M \mathrm{D} \boldsymbol{b}\right\}
$$

and observe that we have the two bounds

$$
\|\phi\|_{L_{t x}^{\infty}} \leqslant \frac{\|\boldsymbol{b}\|_{\infty}}{\delta}, \quad\|\| \phi\left\|_{M_{t x}^{1}} \leqslant\right\| M \mathrm{D} \boldsymbol{b} \|_{M_{t x}^{1}} .
$$

We can exploit the interpolation inequality (7.2) and the bounds (7.5), together with the fact that the function $z \mapsto \log z$ is increasing and the function $z \mapsto$ $z[1+\log (c / z)]$ is increasing for $z \leqslant c / \delta$, to obtain

$$
\begin{aligned}
\Phi_{\delta}(t) & \leqslant C\||\phi|\|_{M_{t x}^{1}}\left[1+\log \left(\frac{C\|\phi\|_{L_{t x}^{\infty}}}{\|\phi \mid\|_{M_{t x}^{1}}^{1}}\right)\right] \\
& \leqslant C\|M \mathrm{D} \boldsymbol{b}\| \|_{M_{t x}^{1}}\left[1+\log \left(\frac{C\|\boldsymbol{b}\|_{L_{t x}^{\infty}}}{\delta\|M \mathrm{D} \boldsymbol{b}\|_{M_{t x}^{1}}}\right)\right] .
\end{aligned}
$$

From (7.1) we simply obtain

$$
\|M \mathrm{D} \boldsymbol{b}\|_{M_{t x}^{1}} \leqslant\|\| \mid M \mathrm{D} \boldsymbol{b}\left\|_{M_{x}^{1}}\right\|_{L_{t}^{1}} \leqslant C\|\mathrm{D} \boldsymbol{b}\|_{L_{t}^{1}\left(\mathcal{M}_{x}\right)},
$$

and again using the fact that the function $z \mapsto z[1+\log (c / z)]$ is increasing for $z \leqslant c / \delta$, this gives

$$
\Phi_{\delta}(t) \leqslant C\|\mathrm{D} \boldsymbol{b}\|_{L_{t}^{1}\left(\mathcal{M}_{x}\right)}\left[1+\log \left(\frac{C}{\delta\|\mathrm{D} \boldsymbol{b}\|_{L_{t}^{1}\left(\mathcal{M}_{x}\right)}}\right)\right],
$$

in which $\|\boldsymbol{b}\|_{\infty}$ has been absorbed in the constant.

This upper bound is exactly on the critical scale discriminating for uniqueness in (6.3)! This means that (7.6) (valid even for BV vector fields) is not enough to conclude.

However, in the $W^{1,1}$ case we can 'play with the constants'. Indeed, given $\varepsilon>0$, the derivative $\nabla \boldsymbol{b} \in L^{1}\left([0, T] \times \mathbb{R}^{d}\right)$ can be split as

$$
\nabla \boldsymbol{b}=g_{\varepsilon}^{1}+g_{\varepsilon}^{2},
$$

with $\left\|g_{\varepsilon}^{1}\right\|_{L_{t}^{1}\left(L_{x}^{1}\right)} \leqslant \varepsilon$ and $\left\|g_{\varepsilon}^{2}\right\|_{L_{t}^{1}\left(L_{x}^{2}\right)} \leqslant C_{\varepsilon}$. The constant $C_{\varepsilon}$ blows up as $\varepsilon \rightarrow 0$ and depends on the equi-integrability of $\nabla \boldsymbol{b}$. Using this argument, we are only able to 
proceed in the $W^{1,1}$ case, and the more general BV case (still allowed in (7.6)) has to be abandoned. A decomposition as in (7.7) does not hold for a measure.

With this decomposition we can estimate the function $\phi$ in (7.4) as follows:

$$
\begin{aligned}
\phi(s, x) & \leqslant \min \left\{\frac{\|\boldsymbol{b}\|_{\infty}}{\delta} ; M g_{\varepsilon}^{1}+M g_{\varepsilon}^{2}\right\} \\
& \leqslant \min \left\{\frac{\|\boldsymbol{b}\|_{\infty}}{\delta} ; M g_{\varepsilon}^{1}\right\}+\min \left\{\frac{\|\boldsymbol{b}\|_{\infty}}{\delta} ; M g_{\varepsilon}^{2}\right\} \\
& =\phi^{1}(s, x)+\phi^{2}(s, x) .
\end{aligned}
$$

Reasoning as before and exploiting (7.7), we have

$$
\begin{gathered}
\left\|\phi^{1}\right\|_{L_{t x}^{\infty}} \leqslant \frac{\|\boldsymbol{b}\|_{\infty}}{\delta}, \\
\left\|\phi^{1}\right\|_{M_{t x}^{1}} \leqslant\left\|M g_{\varepsilon}^{1}\right\|_{M_{t x}^{1}} \leqslant\|\| M g_{\varepsilon}^{1}\left\|_{M_{x}^{1}}\right\|_{L_{t}^{1}} \leqslant C\left\|g_{\varepsilon}^{1}\right\|_{L_{t}^{1}\left(L_{x}^{1}\right)} \leqslant C \varepsilon
\end{gathered}
$$

and

$$
\left\|\phi_{\varepsilon}^{2}\right\|_{L_{t}^{1}\left(L_{x}^{2}\right)} \leqslant C\left\|g_{\varepsilon}^{2}\right\|_{L_{t}^{1}\left(L_{x}^{2}\right)} \leqslant C C_{\varepsilon} .
$$

We use these two estimates in the bound for $\Phi_{\delta}$. The 'borderline' estimate (7.6) is used with the term $g_{\varepsilon}^{1}$, while the term $g_{\varepsilon}^{2}$ is treated according to the analysis for the case $p>1$ in $\S 6.3$. We finally get an estimate of the form

$$
\mathcal{L}^{d}\left(B_{R} \cap\left\{\left|\boldsymbol{X}_{1}-\boldsymbol{X}_{2}\right|>\gamma\right\}\right) \leqslant C \varepsilon \frac{1+\log (C / \delta \varepsilon)}{\log (1+\gamma / \delta)}+\frac{C C_{\varepsilon}}{\log (1+\gamma / \delta)}
$$

and we conclude by choosing first $\varepsilon$ and then $\delta$ sufficiently small.

REMARK 7.2. The extension of this proof to the BV case is an important open problem. This would solve the compactness conjecture proposed by Bressan in [45].

\subsection{An extension to a case involving singular integrals}

We want to extend the argument in $\S 7.2$ to a more general case involving singular integrals of $L^{1}$ functions. We start by presenting a motivation for this setting.

The Euler equation in vorticity form in two dimensions reads

$$
\partial_{t} \omega+\operatorname{div}(\boldsymbol{v} \omega)=0 .
$$

This is a continuity equation for the fluid vorticity $\omega$, which is transported by the (divergence-free) fluid velocity $\boldsymbol{v}$. This is indeed a nonlinear continuity equation due to the structural condition $\omega=\operatorname{curl} \boldsymbol{v}$. Both the vorticity and the velocity are unknowns of the problem, and the coupling can be rewritten as a convolution:

$$
\boldsymbol{v}=K * \omega .
$$

In the above formula, the Biot-Savart kernel is defined (up to multiplicative constants) by $K(x)=x^{\perp} /|x|^{2}$.

In order to proceed with the analysis of (7.8), it is necessary to understand the regularity of the fluid velocity $\boldsymbol{v}$. A formal differentiation of the convolution product in (7.9) gives $\mathrm{D} \boldsymbol{v}=D K * \omega$. The problem now is that $|\mathrm{D} K| \sim 1 /|x|^{2}$ around the 
origin in $\mathbb{R}^{2}$, hence it is in general not even locally integrable. We cannot give a meaning to the formula for the derivative of $\boldsymbol{v}$ as a usual convolution of distributions.

However, the actual expression of $\mathrm{DK}$ (without the modulus!) retains enough cancellations to allow for a definition of the 'convolution' in the sense of CalderónZygmund singular integrals (see [98] for a detailed description of this topic).

The analysis of $\S 7.2$ extends naturally to the case in which the derivative of the vector field $\boldsymbol{b}$ is given by singular integrals of $L^{1}$ functions:

$$
\partial_{j} \boldsymbol{b}^{i}=\sum_{k} S_{i j k} g_{i j k}
$$

where $g_{i j k} \in L^{1}$ and each $S_{i j k}$ is a singular integral operator, associated with a kernel $K_{i j k} \in \mathcal{S}^{\prime}\left(\mathbb{R}^{d}\right) \cap C^{1}\left(\mathbb{R}^{d} \backslash\{0\}\right)$, satisfying

$$
|K(x)| \leqslant \frac{C}{|x|^{d}}, \quad|\mathrm{D} K(x)| \leqslant \frac{C}{|x|^{d+1}}
$$

and the cancellation property

$$
\left|\int_{R_{1}<|x|<R_{2}} K(x) \mathrm{d} x\right| \leqslant C \quad \forall 0<R_{1}<R_{2}<\infty .
$$

REMARK 7.3. This class of vector field is motivated by the example of the twodimensional Euler equation with $L^{1}$ vorticity. It includes $W^{1,1}$, but is strictly bigger; on the other hand, it is not included in, nor does it include, BV.

We now describe the main ideas from this proof. Analogously to maximal functions, singular integrals satisfy strong estimates

$$
\|S f\|_{L^{p}} \leqslant C_{p}\|f\|_{L^{p}}
$$

for every $1<p<\infty$, but only the weak estimate

$$
\|S f\|_{M^{1}} \leqslant C_{1}\|f\|_{L^{1}}
$$

when $p=1$. Given two singular integral operators $S_{1}$ and $S_{2}$, associated with regular enough singular kernels $K_{1}$ and $K_{2}$, the composition $S=S_{2} \circ S_{1}$ is again a singular integral operator, associated with the singular kernel $K=K_{2} * K_{1}$. This means that the weak estimate

$$
\|S f\|_{M^{1}}=\left\|S_{2} \circ S_{1} f\right\|_{M^{1}} \leqslant C_{1}\|f\|_{L^{1}}
$$

holds. Note carefully that this estimate cannot be obtained by composing the two weak estimates, valid for $S_{1}$ and $S_{2}$, from $L^{1}$ into $M^{1}$ separately. The idea is that cancellations in the formal convolution product $K_{2} * K_{1}$ are exploited.

We return to our uniqueness argument that involves the search for upper bounds for the functional $\Phi_{\delta}$. We would like to obtain something similar to (7.6) (with $\|g\|_{L^{1}}$ instead of $\|D \boldsymbol{b}\|_{L^{1}}$ on the right-hand side). We realize that we need an estimate of the form

$$
\|M D \boldsymbol{b}\|_{M^{1}}=\|M S g\|_{M^{1}} \leqslant C\|g\|_{L^{1}} .
$$

The classical maximal function in (6.4) is 'too rough' to allow for such an estimate. The idea is that we can choose a 'smooth maximal function', with absolute value 
outside the integral, and with a smooth weight. More precisely, given $\rho \in C_{\mathrm{c}}^{\infty}$, we define

$$
M_{\rho} f(x)=\sup _{r>0}\left|\frac{1}{r^{d}} \int_{\mathbb{R}^{d}} \rho\left(\frac{x-y}{r}\right) f(y) \mathrm{d} y\right| .
$$

It can be proven that this smooth maximal function (also known as the grand maximal function in the context of Hardy spaces) is still suitable for the estimate of difference quotients as in (6.9). Moreover, the cancellation properties of the kernel $\rho$ play together with those of the singular integral $S$, allowing us to get estimate (7.10). We refer the reader to [37] for the exact statements and for the proofs.

\section{Some applications}

\subsection{A system of conservation laws}

Let us consider the Cauchy problem (studied in one space dimension by KeyfitzKranzer in [82])

$$
\frac{\mathrm{d}}{\mathrm{d} t} u+\sum_{i=1}^{d} \frac{\partial}{\partial x_{i}}\left(\boldsymbol{f}_{i}(|u|) u\right)=0, \quad u: \mathbb{R}^{d} \times(0,+\infty) \rightarrow \mathbb{R}^{k},
$$

with the initial condition $u(\cdot, 0)=\bar{u}$. Here $\boldsymbol{f}: \mathbb{R} \rightarrow \mathbb{R}^{d}$ is a $C^{1}$ function.

In [46], Bressan showed that the problem can be ill posed for $L^{\infty}$ initial data and he conjectured that it could be well posed for BV initial data, suggesting the extension of the classical method of characteristics to this case. In [12] it was proved that this procedure can really be implemented, thanks to the results in [9], for initial data $\bar{u}$ such that $\bar{\rho}:=|\bar{u}| \in \mathrm{BV} \cap L^{\infty}$, with $1 /|\bar{u}| \in L^{\infty}$. Later on it was proved [17] that the lower bound on $\bar{\rho}$ is not necessary and, moreover, that the solution built in [12] is unique in a suitable class of admissible functions: those whose modulus $\rho$ satisfies the scalar PDE

$$
\frac{\mathrm{d}}{\mathrm{d} t} \rho+\sum_{i=1}^{d} \frac{\partial}{\partial x_{i}}\left(\boldsymbol{f}_{i}(\rho) \rho\right)=0
$$

in the Kruzhkov sense (i.e. $\eta(\rho)_{t}+\mathrm{D}_{x} \cdot(\boldsymbol{q}(\rho)) \leqslant 0$ for any convex entropy-entropy flux pair $(\eta, \boldsymbol{q})$; here $(s \boldsymbol{f})^{\prime}(s) \eta^{\prime}(s)=\boldsymbol{q}^{\prime}(s)$. See [65] for existence, uniqueness and regularity results for Kruzhkov solutions), with the initial condition $\rho(0, \cdot)=\bar{\rho}$.

Notice that the regularity theory for this class of solutions gives that $\rho \in L^{\infty} \cap$ $\mathrm{BV}_{\text {loc }}\left([0,+\infty) \times \mathbb{R}^{d}\right)$ due to the BV regularity and the boundedness of $|\bar{u}|$. Furthermore, the maximum principle gives $0<1 / \rho \leqslant 1 /|\bar{u}| \in L^{\infty}$.

In order to obtain the (or, better, a) solution $u$ we can formally decouple the system, writing

$$
u=\theta \rho, \quad \bar{u}=\bar{\theta} \bar{\rho}, \quad|\theta|=|\bar{\theta}|=1,
$$

thus reducing the problem to the system (decoupled, if one neglects the constraint $|\theta|=1$ ) of transport equations

$$
\theta_{t}+\sum_{i=1}^{d} \frac{\partial}{\partial x_{i}}\left(\boldsymbol{f}_{i}(\rho) \theta\right)=0
$$

with the initial condition $\theta(0, \cdot)=\bar{\theta}$. 
A formal solution of the system, also satisfying the constraint $|\theta|=1$, is given by

$$
\theta(t, x):=\bar{\theta}\left([\boldsymbol{X}(t, \cdot)]^{-1}(x)\right),
$$

where $\boldsymbol{X}(t, \cdot)$ is the flow associated with $\boldsymbol{f}(\rho)$. Notice that the non-autonomous vector field $\boldsymbol{f}(\rho)$ is bounded and of class $\mathrm{BV}_{\text {loc }}$, but the theory illustrated in this paper is not immediately applicable because its divergence is not absolutely continuous with respect to $\mathcal{L}^{d+1}$. In this case, however, a simple argument still allows the use of the theory, representing $\boldsymbol{f}(\rho)$ as a part of the autonomous vector field $\boldsymbol{b}:=(\rho, \rho \boldsymbol{f}(\rho))$ in $\mathbb{R}^{+} \times \mathbb{R}^{d}$. This new vector field is still $\mathrm{BV}_{\text {loc }}$ and bounded, and it is divergence free due to $(8.2)$.

At this point, it is not hard to see that the re-parametrization of the flow $(t(s), \boldsymbol{x}(s))$ associated with $\boldsymbol{b}$

$$
(\dot{t}(s), \dot{\boldsymbol{x}}(s))=(\rho(t(s), \boldsymbol{x}(s)), \boldsymbol{f}(\rho(t(s), \boldsymbol{x}(s))) \rho(t(s), \boldsymbol{x}(s)))
$$

defined by $\tilde{\boldsymbol{x}}(t)=\boldsymbol{x}\left(t(s)^{-1}(t)\right)$ (and here we use the assumption $\rho>0$ ) defines a flow for the vector field $\boldsymbol{f}(\rho)$ we were originally interested in.

In this way we get a kind of formal, or pointwise, solution of the system (8.2) that could in principle be very far from being a distributional solution. But here the stability theorem comes into play, showing that all formal computations above can be justified just first by assuming $(\rho, \boldsymbol{f}(\rho))$ smooth, and then by approximation (see [12] for details).

\subsection{Lagrangian solutions of semi-geostrophic equations}

The semi-geostrophic equations are a simplified model of the atmosphere/ocean flows [62] described by the system of transport equations

$$
\left.\begin{array}{l}
\frac{\mathrm{d}}{\mathrm{d} t} \partial_{2} p+\boldsymbol{u} \cdot \nabla \partial_{2} p=-\boldsymbol{u}_{2}+\partial_{1} p, \\
\frac{\mathrm{d}}{\mathrm{d} t} \partial_{1} p+\boldsymbol{u} \cdot \nabla \partial_{1} p=-\boldsymbol{u}_{1}-\partial_{2} p, \\
\frac{\mathrm{d}}{\mathrm{d} t} \partial_{3} p+\boldsymbol{u} \cdot \nabla \partial_{3} p=0 .
\end{array}\right\}
$$

Here the velocity $\boldsymbol{u}$ is a divergence-free field, $p$ is the pressure and $\rho:=-\partial_{3} p$ represents the density of the fluid. We consider the problem in $[0, T] \times \Omega$, with $\Omega$ bounded and convex. Initial conditions are given on the pressure and a no-flux through $\partial \Omega$ condition is imposed for all times.

By introducing the modified pressure $P_{t}(x):=p_{t}(x)+\frac{1}{2}\left(x_{1}^{2}+x_{2}^{2}\right)$, (SGE) can be written in a more compact form as

$$
\frac{\mathrm{d}}{\mathrm{d} t} \nabla P_{t}+\boldsymbol{u} \cdot \nabla^{2} P_{t}=J\left(\nabla P_{t}-x\right) \quad \text { with } J:=\left(\begin{array}{ccc}
0 & -1 & 0 \\
1 & 0 & 0 \\
0 & 0 & 0
\end{array}\right) .
$$

The existence of solutions for this problem was an open problem until very recently, while uniqueness remains open. In $[28,64]$, existence results have been obtained in the so-called dual coordinates, where we replace the physical variable $x$ by $X=$ 
$\nabla P_{t}(x)$. Under this change of variables, and assuming $P_{t}$ to be convex, the system becomes

$$
\frac{\mathrm{d}}{\mathrm{d} t} \alpha_{t}+\mathrm{D}_{x} \cdot\left(\boldsymbol{U}_{t} \alpha_{t}\right)=0 \quad \text { with } \boldsymbol{U}_{t}(X):=J\left(X-\nabla P_{t}^{*}(X)\right)
$$

with $\alpha_{t}:=\left(\nabla P_{t}\right)_{\#}\left(\mathcal{L}_{\Omega}\right)$ (here we denote by $\mathcal{L}_{\Omega}$ the restriction of $\mathcal{L}^{d}$ to $\Omega$ ). Indeed, for any test function $\varphi$ we can use the fact that $\boldsymbol{u}$ is divergence free to obtain:

$$
\begin{aligned}
\frac{\mathrm{d}}{\mathrm{d} t} \int_{\mathbb{R}^{d}} \varphi \mathrm{d} \alpha_{t} & =\int_{\mathbb{R}^{d}} \nabla \varphi\left(\nabla P_{t}\right) \cdot \frac{\mathrm{d}}{\mathrm{d} t} \nabla P_{t} \mathrm{~d} x \\
& =\int_{\mathbb{R}^{d}} \nabla \varphi\left(\nabla P_{t}\right) \cdot J\left(\nabla P_{t}-x\right) \mathrm{d} x+\int_{\mathbb{R}^{d}} \nabla \varphi\left(\nabla P_{t}\right) \nabla^{2} P_{t} \cdot \boldsymbol{u} \mathrm{d} x \\
& =\int_{\mathbb{R}^{d}} \nabla \varphi \cdot J\left(X-\nabla P_{t}^{*}\right) \mathrm{d} \alpha_{t}+\int_{\mathbb{R}^{d}} \nabla\left(\varphi \circ \nabla P_{t}\right) \cdot \boldsymbol{u} \mathrm{d} x \\
& =\int_{\mathbb{R}^{d}} \nabla \varphi \cdot \boldsymbol{U}_{t} \mathrm{~d} \alpha_{t} .
\end{aligned}
$$

Existence of a solution to (8.5) can be obtained by a suitable time discretization scheme. Now the question is, can we go back to the original physical variables? An important step forward has been achieved, by Cullen and Feldman [63], with the concept of a Lagrangian solution of (SGE).

Taking into account that the vector field $\boldsymbol{U}_{t}(X)=J\left(X-\nabla P_{t}^{*}(X)\right)$ is $\mathrm{BV}$, bounded and divergence free, there is a well-defined stable and measure-preserving flow $\boldsymbol{X}(t, X)=\boldsymbol{X}_{t}(X)$ relative to $\boldsymbol{U}$. This flow can be carried back to the physical space with the transformation

$$
F_{t}(x):=\nabla P_{t}^{*} \circ \boldsymbol{X}_{t} \circ \nabla P_{0}(x),
$$

thus defining maps $F_{t}$ preserving $\mathcal{L}_{\Omega}^{d}$.

Using the stability theorem, one can also show that $Z_{t}(x):=\nabla P_{t}\left(F_{t}(x)\right)$ solve, in the distributions sense, the Lagrangian form of (8.4), i.e.

$$
\frac{\mathrm{d}}{\mathrm{d} t} Z_{t}(x)=J\left(Z_{t}-F_{t}\right) .
$$

This provides us with a sort of weak solution of (8.4). In connection with existence of solutions to the semi-geostrophic problem in the original physical variables, a formal argument suggests that, given $P_{t}$, the velocity $\boldsymbol{u}$ should be defined by

$$
\partial_{t} \nabla P_{t}^{*}\left(\nabla P_{t}(x)\right)+\nabla^{2} P_{t}^{*}\left(\nabla P_{t}(x)\right) J\left(\nabla P_{t}(x)-x\right) .
$$

On the other hand, the a priori regularity on $\nabla P_{t}$ (ensured by the convexity of $P_{t}$ ) is a BV regularity. In more recent times, distributional solutions for the Eulerian form of the PDE have been recovered [23,24] using refined regularity results on Alexandrov solutions to Monge-Ampère equations that provide not only BV regularity but also Sobolev regularity (see $[68,69,96]$, improving earlier results in $[47-50,101,102]$ in the borderline case when the right-hand side is bounded away from 0 and $\infty$, but only Borel). 


\section{Open problems, bibliographical notes and references}

Section 2. The material contained in this section is classical. Good references are [74], [22, ch. 8], [39] and [72]. For the proof of the area formula, see, for instance, $[16,77,78]$.

The proof of the second local variant, under the stronger assumption

$$
\int_{0}^{T} \int_{\mathbb{R}^{d}}\left|\boldsymbol{b}_{t}\right| \mathrm{d} \mu_{t} \mathrm{~d} t<+\infty,
$$

is given in [22, proposition 8.1.8]. The same proof works under the weaker assumption (2.7).

Section 3. Many ideas of this section, and in particular the idea of looking at measures in the space of continuous maps to characterize the flow and prove its stability, are borrowed from [9], which deals with BV vector fields. Later on, the arguments have been put in a more general form, independent of the specific class of vector fields under consideration (see [10]). See also [11], which provides more insight into the quantitative side of the theory and the differentiability properties of the flow.

The idea of a probabilistic representation is of course classical, and appears in many contexts (particularly for equations of diffusion type). To the best of our knowledge the first reference in the context of conservation laws and fluid mechanics is [41], where a similar approach is proposed for the incompressible Euler equation (see also [42-44]): in this case the compact (but neither metrizable, nor separable) space $X^{[0, T]}$, with $X \subset \mathbb{R}^{d}$ compact, was considered.

This approach is by now a familiar one in optimal transport theory, where transport maps and transference plans can be thought of in a natural way as measures in the space of minimizing geodesics [95], and in the so-called irrigation problems, a nice variant of the optimal transport problem [30]. See also [27] for a similar approach within Mather's theory. The lecture notes [105] (see also the appendix of [88]) contain, among other things, a comprehensive treatment of the topic of measures in the space of action-minimizing curves, including at the same time the optimal transport and the dynamical systems case (this unified treatment was inspired by [29]). Another related reference is [67].

The superposition principle is proved under the weaker assumption

$$
\int_{0}^{T} \int_{\mathbb{R}^{d}}\left|\boldsymbol{b}_{t}\right|^{p} \mathrm{~d} \mu_{t} \mathrm{~d} t<+\infty
$$

for some $p>1$, in [22, theorem 8.2.1]. See also [89] for the extension to the case $p=1$ and to the non-homogeneous continuity equation. Very closely related results, relative to the representation of a vector field as the superposition of 'elementary' vector fields associated with curves, appear in [27,97].

In [20] an interesting variant of the stability theorems 3.13 and 4.10 is discussed, peculiar to the case when the limit vector field $\boldsymbol{b}$ is a sufficiently regular gradient. In this case it was proved that weak convergence of $\mu_{t}^{h}$ to $\mu_{t}$ for all $t \in[0, T]$ and 
the energy estimate

$$
\limsup _{h \rightarrow \infty} \int_{0}^{T} \int_{\mathbb{R}^{d}}\left|\boldsymbol{b}_{t}^{h}\right|^{2} \mathrm{~d} \mu_{t}^{h} \mathrm{~d} t \leqslant \int_{0}^{T} \int_{\mathbb{R}^{d}}\left|\boldsymbol{b}_{t}\right|^{2} \mathrm{~d} \mu_{t} \mathrm{~d} t<+\infty
$$

are sufficient to obtain the stability property. This is a consequence of the fact that, for $\mu_{t}$ given, gradient vector fields minimize $\int_{0}^{T} \int\left|\boldsymbol{c}_{t}\right|^{2} \mathrm{~d} \mu_{t}$ among all velocity fields $\boldsymbol{c}_{t}$ for which the continuity equation $\left(\mathrm{d} \mu_{t} / \mathrm{d} t\right)+\mathrm{D}_{x} \cdot\left(\boldsymbol{c}_{t} \mu_{t}\right)=0$ holds (see [22, ch. 8] for a general proof of this fact and for references to earlier works of Otto and Benamou-Brenier).

Section 4. The definition of a renormalized solution and the strong convergence of commutators are entirely borrowed from [72]. See also [73] for the relevance of this concept in connection with the existence theory for the Boltzmann equation.

The differentiability properties of the flow in [83]: later on, this differentiability property was characterized and compared with the more classical approximate differentiability (see [78]) in [15], while [19] contains the proof of the stronger 'local' Lipschitz properties. Theorem 4.11 summarizes all of these results. Also, [61] contains more explicit Lipschitz estimates and an independent proof of the compactness of flows. See [51] for a proof, using radial convolution kernels, of the renormalization property for vector fields satisfying $D_{i} \boldsymbol{b}^{j}+D_{j} \boldsymbol{b}^{i} \in L_{\mathrm{loc}}^{1}$.

Both the method illustrated in this paper and the DiPerna-Lions method are based on abstract compactness arguments and do not provide a rate of convergence in the stability theorem.

A few existence results for Sobolev vector fields seem to be known in the infinitedimensional case; see [31] and the more recent paper [14]. Also, the investigation of non-Euclidean geometries, e.g. Carnot groups and horizontal vector fields, could provide interesting results.

Finally, notice that the theory has a natural invariance. Namely, if $\boldsymbol{X}$ is a flow relative to $\boldsymbol{b}$, then $\boldsymbol{X}$ is a flow relative to $\tilde{\boldsymbol{b}}$ whenever $\{\tilde{\boldsymbol{b}} \neq \boldsymbol{b}\}$ is $\mathcal{L}^{1+d}$-negligible in $(0, T) \times \mathbb{R}^{d}$. So a natural question is whether the uniqueness 'in the selection sense' might be enforced by choosing a canonical representative $\tilde{\boldsymbol{b}}$ in the equivalence class of $\boldsymbol{b}$ : in other words we may think that, for a suitable choice of $\tilde{\boldsymbol{b}}$, the ODE $\dot{\gamma}(t)=\tilde{\boldsymbol{b}}_{t}(\gamma(t))$ has a unique absolutely continuous solution starting from $x$ for $\mathcal{L}^{d}$-a.e. $x$.

Section 5. Here we closely followed [9]. The main idea of this section, i.e. the adaptation of the convolution kernel to the local behaviour of the vector field, was used at various levels of generality $[34,56,86]$ (see also $[52,53]$ for related results independent of this technique) until the general result [9].

The optimal regularity condition on $\boldsymbol{b}$ ensuring the renormalization property, and therefore the well-posedness in $\mathcal{L}_{\boldsymbol{b}}$, is still not known. New results, both in the Sobolev and in the BV framework, are presented in $[18,83,84]$.

As mentioned in remark 4.3, it is proved in [35] that the renormalization property can be characterized in terms of the uniqueness (for both the forward and the backward Cauchy problems) and the strong continuity of weak solutions, or in terms of the density of smooth functions with respect to a suitable 'graph norm'. This in some sense tells us that the renormalization property is more than a 'technical 
tool' to prove uniqueness, but is really a substantial property related to the wellposedness.

In [21], the possibility of proving the renormalization property for nearly incompressible $\mathrm{BV}_{\text {loc }} \cap L^{\infty}$ fields $\boldsymbol{b}$ is investigated: nearly incompressible fields are defined by requiring the existence of a positive function $\rho$, with $\ln \rho \in L^{\infty}$, such that the space-time field $(\rho, \rho \boldsymbol{b})$ is divergence free. As in the case of the Keyfitz-Kranzer system, the existence a function $\rho$ with this property seems to be a natural replacement of the condition $\mathrm{D}_{x} \cdot \boldsymbol{b} \in L^{\infty}$ (and is actually implied by it); as explained in [17], a proof of the renormalization property in this context would lead to the proof of a conjecture, due to Bressan, on the compactness of flows associated with a sequence of vector fields bounded in BV.

The situation in the two-dimensional context is somehow more 'rigid'. In the series of papers [5-7] a characterization of the uniqueness for the continuity equation is proved in the context of two-dimensional bounded autonomous divergence-free vector fields. To any such vector field $b$ a Lipschitz function $f: \mathbb{R}^{2} \rightarrow \mathbb{R}$ so that $b=\nabla^{\perp} f$ can be associated. The main result of [6] is the equivalence between the uniqueness and a 'measure theoretical version' of the Sard property for the function $f$, which, apart from a minor technical detail, reads

$$
f_{\#}\left(\mathcal{L}^{2}\llcorner\{\nabla f=0\}) \perp \mathcal{L}^{1} .\right.
$$

The heart of the proof is a suitable disintegration of the PDE along level sets of $f$. In this way, two-dimensional uniqueness is linked to uniqueness on the level sets. It turns out that (9.1) is the condition characterizing uniqueness for such one-dimensional problems.

Section 6. The presentation in this section is based on [61]. Some related analysis, with further developments in the case of kinetic equations, is contained in $[54,81]$.

Section 7. Here the analysis follows [37], but, in order to make the presentation more accessible, we skipped most of the details and essentially tried to convey the general ideas. A similar presentation can be found in $[36,59]$.

The main open problem in this context is clearly the extension of the quantitative estimates to BV vector fields, or even more to vector fields whose gradient is given by singular integrals of measures (and not just of $L^{1}$ functions). In the work in progress [33], the following split case is addressed: the derivatives with respect to some coordinates are singular integrals of measures, while all other derivatives are singular integrals of $L^{1}$ functions. The strategy is based on an anisotropic version of the functional.

The quantitative estimates in the context of singular integrals are relevant for applications to nonlinear PDEs. In particular, in [32] we plan to study existence and energy concentration for solutions to the two-dimensional Euler equation with $L^{1}$ vorticity, and some existence settings for the Vlasov-Poisson equation.

Section 8. In connection with the Keyfitz-Kranzer system there are several open questions. In particular, one would like to obtain uniqueness (and stability) of the solution in more general classes of admissible functions (partial results in this direction are given in [17]). A strictly related problem is the convergence of the 
vanishing viscosity method to the solution built in [12]. Also, very little about the regularity of solutions is presently known: we know [66] that BV estimates do not hold and, besides, that the construction in [12] seems not to be applicable to more general systems of triangular type; see the counter-example in [60].

\section{Acknowledgements}

This paper is based on lecture notes that were written on the occasion of a crash course given by the authors at Heriot-Watt University in Edinburgh on April 15-16, 2013. The authors warmly thank the organizers for the invitation and for the kind hospitality. They also thank Anna Bohun for a careful reading of a preliminary version of these notes.

\section{References}

1 M. Aizenman. On vector fields as generators of flows: a counterexample to Nelson's conjecture. Annals Math. 107 (1978), 287-296.

2 G. Alberti. Rank-one properties for derivatives of functions with bounded variation. Proc. R. Soc. Edinb. A 123 (1993), 239-274.

3 G. Alberti and L. Ambrosio. A geometric approach to monotone functions in $\mathbb{R}^{n}$. Math. Z. 230 (1999), 259-316.

4 G. Alberti and S. Müller. A new approach to variational problems with multiple scales. Commun. Pure Appl. Math. 54 (2001), 761-825.

5 G. Alberti, S. Bianchini and G. Crippa. Structure of level sets and Sard-type properties of Lipschitz maps: results and counterexamples. Annali Scuola Norm. Sup. Pisa IV 12 (2013), 863-902.

6 G. Alberti, S. Bianchini and G. Crippa. A uniqueness result for the continuity equation in two dimensions. J. Eur. Math. Soc. 16 (2014), 201-234.

7 G. Alberti, S. Bianchini and G. Crippa. On the $L^{p}$ differentiability of certain classes of functions. Rev. Mat. Iber. 30 (2014), 349-367.

8 F. J. Almgren. The theory of varifolds: a variational calculus in the large (Princeton University Press, 1972).

9 L. Ambrosio. Transport equation and Cauchy problem for BV vector fields. Invent. Math. 158 (2004), 227-260.

10 L. Ambrosio. Transport equation and Cauchy problem for non-smooth vector fields. In Calculus of variations and non-linear partial differential equations (ed. B. Dacorogna and P. Marcellini). Lecture Notes in Mathematics, vol. 1927, pp. 1-41 (Springer, 2008).

11 L. Ambrosio and G. Crippa. Existence, uniqueness, stability and differentiability properties of the flow associated to weakly differentiable vector fields. In Transport equations and multi-D hyperbolic conservation laws. Lecture Notes of the Unione Matematica Italiana, vol. 5, pp. 3-57 (Springer, 2008).

12 L. Ambrosio and C. De Lellis. Existence of solutions for a class of hyperbolic systems of conservation laws in several space dimensions. Int. Math. Res. Not. 41 (2003), 2205-2220.

13 L. Ambrosio and A. Figalli. Geodesics in the space of measure-preserving maps and plans. Arch. Ration. Mech. Analysis 194 (2009), 421-462.

14 L. Ambrosio and A. Figalli. On flows associated to Sobolev vector fields in Wiener spaces: an approach à la DiPerna-Lions. J. Funct. Analysis 256 (2009), 179-214.

15 L. Ambrosio and J. Malý. Very weak notions of differentiability. Proc. R. Soc. Edinb. A 137 (2007), 447-455.

16 L. Ambrosio, N. Fusco and D. Pallara. Functions of bounded variation and free discontinuity problems. Oxford Mathematical Monographs (Oxford University Press, 2000).

17 L. Ambrosio, F. Bouchut and C. De Lellis. Well-posedness for a class of hyperbolic systems of conservation laws in several space dimensions. Commun. PDEs 29 (2004), 1635-1651.

18 L. Ambrosio, G. Crippa and S. Maniglia. Traces and fine properties of a $B D$ class of vector fields and applications. Annales Fac. Sci. Toulouse Math. 14 (2005), 527-561. 
L. Ambrosio, M. Lecumberry and S. Maniglia. Lipschitz regularity and approximate differentiability of the DiPerna-Lions flow. Rend. Sem. Mat. Univ. Padova 114 (2005), 29-50. and convergence of iterated transport maps. Manuscr. Math. 121 (2006), 1-50.

21 L. Ambrosio, C. De Lellis and J. Malý. On the chain rule for the divergence of BV like vector fields: applications, partial results, open problems. In Perspectives in nonlinear partial differential equations: in honour of Haim Brézis. Contemporary Mathematics, vol. 446, pp. 31-67 (Providence, RI: American Mathematical Society, 2007).

Ambrosio, N. Gigli and G. Savaré. Gradient flows in metric spaces and in the Wasser stein space of probability measures, 2nd edn. Lectures in Mathematics ETH Zurich (Birkhäuser, 2008)

23 L. Ambrosio, M. Colombo, G. De Philippis and A. Figalli. Existence of Eulerian solutions to the semigeostrophic equations in physical space: the two-dimensional periodic case. Commun. PDEs 37 (2012), 2209-2227.

24 L. Ambrosio, M. Colombo, G. De Philippis and A. Figalli. A global existence result for the semigeostrophic equations in three dimensional convex domains. Preprint, 2012. (Available at http://arxiv.org/abs/1205.5435.)

25 E. J. Balder. New fundamentals of Young measure convergence. In Calculus of variations and optimal control, Chapman and Hall/CRC Research Notes in Mathematics, vol. 410, pp. 24-48 (Boca Raton, FL: CRC Press, 1999).

26 J. Ball and R. James. Fine phase mixtures as minimizers of energy. Arch. Ration. Mech. Analysis 100 (1987), 13-52.

27 V. Bangert. Minimal measures and minimizing closed normal one-currents. Geom. Funct. Analysis 9 (1999), 413-427.

28 J.-D. Benamou and Y. Brenier. Weak solutions for the semigeostrophic equation formulated as a couples Monge-Ampère transport problem. SIAM J. Appl. Math. 58 (1998), 14501461.

29 P. Bernard and B. Buffoni. Optimal mass transportation and Mather theory. J. Eur. Math. Soc. 1 (2007), 85-121.

30 M. Bernot, V. Caselles and J. M. Morel. Traffic plans. Publ. Mat. 49 (2005), 417-451.

31 V. Bogachev and E. M. Wolf. Absolutely continuous flows generated by Sobolev class vector fields in finite and infinite dimensions. J. Funct. Analysis 167 (1999), 1-68.

32 A. Bohun, F. Bouchut and G. Crippa. Lagrangian solutions to the Vlasov-Poisson equation with $L^{1}$ density. (In preparation.)

33 A. Bohun, F. Bouchut and G. Crippa. Lagrangian flows for vector fields with anisotropic regularity. (In preparation.)

34 F. Bouchut. Renormalized solutions to the Vlasov equation with coefficients of bounded variation. Arch. Ration. Mech. Analysis 157 (2001), 75-90.

35 F. Bouchut and G. Crippa. Uniqueness, renormalization, and smooth approximations for linear transport equations. SIAM J. Math. Analysis 38 (2006), 1316-1328.

36 F. Bouchut and G. Crippa. Equations de transport à coefficient dont le gradient est donné par une intégrale singulière. In Séminaire: Équations aux Dérivées Partielles, 2007-2008, Exp. I (Palaiseau, France: École Polytechnique, 2009).

37 F. Bouchut and G. Crippa. Lagrangian flows for vector fields with gradient given by a singular integral. J. Hyperbolic Diff. Eqns 10 (2013), 235-282.

38 F. Bouchut and F. James. One dimensional transport equation with discontinuous coefficients. Nonlin. Analysis 32 (1998), 891-933.

39 F. Bouchut, F. Golse and M. Pulvirenti. Kinetic equations and asymptotic theory, Series in Applied Mathematics (Paris: Gauthiers-Villars, 2000).

40 F. Bouchut, F. James and S. Mancini. Uniqueness and weak stability for multi-dimensional transport equations with one-sided Lipschitz coefficients. Annali Scuola Norm. Sup. Pisa 4 (2005), 1-25.

41 Y. Brenier. The least action principle and the related concept of generalized flows for incompressible perfect fluids. J. Am. Math. Soc. 2 (1989), 225-255.

42 Y. Brenier. The dual least action problem for an ideal, incompressible fluid. Arch. Ration. Mech. Analysis 122 (1993), 323-351.

43 Y. Brenier. A homogenized model for vortex sheets. Arch. Ration. Mech. Analysis 138 (1997), 319-353. 
Y. Brenier. Minimal geodesics on groups of volume-preserving maps and generalized solutions of the Euler equations. Commun. Pure Appl. Math. 52 (1999), 411-452.

45 A. Bressan. A lemma and a conjecture on the cost of rearrangements. Rend. Sem. Mat. Univ. Padova 110 (2003), 97-102.

46 A. Bressan. An ill posed Cauchy problem for a hyperbolic system in two space dimensions. Rend. Sem. Mat. Univ. Padova 110 (2003), 103-117.

47 L. A. Caffarelli. Some regularity properties of solutions of Monge-Ampère equation. Commun. Pure Appl. Math. 44 (1991), 965-969.

48 L. A. Caffarelli. Boundary regularity of maps with convex potentials. Commun. Pure Appl. Math. 45 (1992), 1141-1151.

49 L. A. Caffarelli. The regularity of mappings with a convex potential. J. Am. Math. Soc. 5 (1992), 99-104.

50 L. A. Caffarelli. Boundary regularity of maps with convex potentials. Annals Math. 144 (1996), 453-496.

51 I. Capuzzo Dolcetta and B. Perthame. On some analogy between different approaches to first order PDEs with nonsmooth coefficients. Adv. Math. Sci. Appl. 6 (1996), 689-703.

52 A. Cellina. On uniqueness almost everywhere for monotonic differential inclusions. Nonlin. Analysis TMA 25 (1995), 899-903.

53 A. Cellina and M. Vornicescu. On gradient flows. J. Diff. Eqns 145 (1998), 489-501.

54 N. Champagnat and P.-E. Jabin. Well posedness in any dimension for Hamiltonian flows with non BV force terms. Commun. PDEs 35 (2010), 786-816.

55 F. Colombini and N. Lerner. Uniqueness of continuous solutions for BV vector fields. Duke Math. J. 111 (2002), 357-384.

56 F. Colombini and N. Lerner. Uniqueness of $L^{\infty}$ solutions for a class of conormal BV vector fields. Contemp. Math. 368 (2005), 133-156.

57 F. Colombini, T. Luo and J. Rauch. Uniqueness and nonuniqueness for nonsmooth divergence-free transport. In Séminaire: Équations aux Dérivées Partielles, 2002-2003, Exp. XXII (Palaiseau: École Polytechnique, 2003).

58 G. Crippa. The flow associated to weakly differentiable vector fields. Theses (Scuola Normale Superiore), vol. 12 (Pisa: Edizioni della Normale, 2009).

59 G. Crippa. Ordinary differential equations and singular integrals. In Hyperbolic problems: theory, numerics, applications. Proc. Int. Conf. on Hyperbolic Problems, HYP2012, Padova, June 24-29, 2012 (ed. F. Ancona, A. Bressan, P. Marcati and A. Marson). American Institute of Mathematical Sciences Book Series on Applied Mathematics, vol. 8, pp. 108-117 (Springfield, MO: American Institute of Mathematical Sciences, 2014)

60 G. Crippa and C. De Lellis. Oscillatory solutions to transport equations. Indiana Univ. Math. J. 55 (2006), 1-13.

61 G. Crippa and C. De Lellis. Estimates for transport equations and regularity of the DiPerna-Lions flow. J. Reine Angew. Math. 616 (2008), 15-46.

62 M. Cullen. On the accuracy of the semi-geostrophic approximation. Q. J. R. Meteorol. Soc. 126 (2000), 1099-1115.

63 M. Cullen and M. Feldman. Lagrangian solutions of semigeostrophic equations in physical space. J. Math. Analysis 37 (2006), 1371-1395.

64 M. Cullen and W. Gangbo. A variational approach for the 2-dimensional semi-geostrophic shallow water equations. Arch. Ration. Mech. Analysis 156 (2001), 241-273.

65 C. Dafermos. Hyperbolic conservation laws in continuum physics (Springer, 2000).

66 C. De Lellis. Blow-up of the BV norm in the multidimensional Keyfitz and Kranzer system. Duke Math. J. 127 (2004), 313-339.

67 L. De Pascale, M. S. Gelli and L. Granieri. Minimal measures, one-dimensional currents and the Monge-Kantorovich problem. Calc. Var. PDEs 27 (2006), 1-23.

68 G. De Philippis and A. Figalli. $W^{2,1}$ regularity for solutions to the Monge-Ampère equation. Invent. Math. 192 (2013), 55-69.

69 G. De Philippis, A. Figalli and O. Savin. A note on $W^{2,1+\varepsilon}$ interior regularity for solutions to the Monge-Ampère equation. Math. Annalen 357 (2013), 11-22.

70 N. Depauw. Non unicitédes solutions bornées pour un champ de vecteurs BV en dehors d'un hyperplan. C. R. Acad. Sci. Paris I 337 (2003), 249-252.

71 R. J. DiPerna. Measure-valued solutions to conservation laws. Arch. Ration. Mech. Analysis 88 (1985), 223-270. 
R. J. DiPerna and P.-L. Lions. Ordinary differential equations, transport theory and Sobolev spaces. Invent. Math. 98 (1989), 511-547.

R. J. DiPerna and P.-L. Lions. On the Cauchy problem for the Boltzmann equation: global existence and weak stability. Annals Math. 130 (1989), 312-366.

C. Evans. Partial differential equations. Graduate Studies in Mathematics, vol. 19 (Providence, RI: American Mathematical Society, 1998).

L. C. Evans. Partial differential equations and Monge-Kantorovich mass transfer. In Current Developments in Mathematics, 1997, pp. 65-126 (Somerville, MA: International Press, 1999).

L. C. Evans and W. Gangbo. Differential equations methods for the Monge-Kantorovich mass transfer problem. Memoirs of the American Mathematical Society, vol. 653 (Providence, RA: American Mathematical Society, 1999).

L. C. Evans and R. F. Gariepy. Lecture notes on measure theory and fine properties of functions (CRC Press, 1992).

H. Federer. Geometric measure theory (Springer, 1969).

M. Hauray. On two-dimensional Hamiltonian transport equations with $L_{\mathrm{loc}}^{p}$ coefficients. Annales Inst. H. Poincaré Analyse Non Linéaire 20 (2003), 625-644.

M. Hauray. On Liouville transport equation with potential in $\mathrm{BV}_{\text {loc }}$. Commun. PDEs 29 (2004), 207-217.

P.-E. Jabin. Differential equations with singular fields. J. Math. Pures Appl. 94 (2010), 597-621.

B. L. Keyfitz and H. C. Kranzer. A system of nonstrictly hyperbolic conservation laws arising in elasticity theory. Arch. Ration. Mech. Analysis 72 (1980), 219-241.

C. Le Bris and P.-L. Lions. Renormalized solutions of some transport equations with partially $W^{1,1}$ velocities and applications. Annali Mat. 183 (2003), 97-130.

N. Lerner. Transport equations with partially BV velocities. Annali Scuola Norm. Sup. Pisa 3 (2004), 681-703.

P.-L. Lions. Mathematical topics in fluid mechanics, Vol. I: incompressible models. Oxford Lecture Series in Mathematics and Its Applications, vol. 3 (Oxford University Press, 1996). P.-L. Lions. Sur les équations différentielles ordinaires et les équations de transport. $C . R$. Acad. Sci. Paris I 326 (1998), 833-838.

P.-L. Lions. Mathematical topics in fluid mechanics, Vol. II: compressible models. Oxford Lecture Series in Mathematics and Its Applications, vol. 10 (Oxford University Press, 1998). Analysis 245 (2007), 311-333.

S. Maniglia. Probabilistic representation and uniqueness results for measure-valued solutions of transport equations. J. Math. Pures Appl. 87 (2007), 601-626.

J. N. Mather. Minimal measures. Comment. Math. Helv. 64 (1989), 375-394. tems. Math. Z. 207 (1991), 169-207.

E. Y. Panov. On strong precompactness of bounded sets of measure-valued solutions of a first order quasilinear equation. Mat. Sb. 186 (1995), 729-740.

G. Petrova and B. Popov. Linear transport equation with discontinuous coefficients. Commun. PDEs 24 (1999), 1849-1873.

F. Poupaud and M. Rascle. Measure solutions to the linear multidimensional transport equation with non-smooth coefficients. Commun. PDEs 22 (1997), 337-358.

A. Pratelli. Equivalence between some definitions for the optimal transport problem and for the transport density on manifolds. Annali Mat. Pura Appl. 184 (2005), 215-238.

T. Schmidt. $W^{1,2+\varepsilon}$ estimates for solutions to the Monge-Ampère equation. Adv. Math. 240 (2013), 672-689.

S. K. Smirnov. Decomposition of solenoidal vector charges into elementary solenoids and the structure of normal one-dimensional currents. St Petersburg Math. J. 5 (1994), 841-867. E. Stein. Singular integrals and differentiability properties of functions (Princeton University Press, 1970).

L. Tartar. Compensated compactness and applications to partial differential equations. In Research Notes in Mathematics, Nonlinear Analysis and Mechanics (ed. R. J. Knops), vol. 4, pp. 136-211 (Boston, MA: Pitman Press, 1979). 
100 R. Temam. Problémes mathématiques en plasticité (Paris: Gauthier-Villars, 1983).

101 J. I. E. Urbas. Global Hölder estimates for equations of Monge-Ampère type. Invent. Math. 91 (1988), 1-29.

102 J. I. E. Urbas. Regularity of generalized solutions of Monge-Ampère equations. Math. Z. 197 (1988), 365-393.

103 A. Vasseur. Strong traces for solutions of multidimensional scalar conservation laws. Arch. Ration. Mech. Analysis 160 (2001), 181-193.

104 C. Villani. Topics in mass transportation. Graduate Studies in Mathematics, vol. 58 (Providence, RI: American Mathematical Society, 2004).

105 C. Villani. Optimal transport: old and new. Grundlehren der Mathematischen Wissenschaften, vol. 338 (Springer, 2009).

106 L. C. Young. Lectures on the calculus of variations and optimal control theory (Philadelphia, PA: Saunders, 1969).

(Issued 5 December 2014) 